\title{
AN INVESTIGATION OF THE MECHANICAL AND BIOLOGICAL PROPERTIES OF NYLON COATED ULTRA-HIGH-MOLECULAR-WEIGHT POLYETHYLENE
}

by

\section{Dariush Firouzi}

Master of Science in Mechanical Engineering, Eastern Mediterranean University (2007)

Bachelor of Science in Materials Engineering, Shiraz University (2005)

\author{
A dissertation presented to \\ RYERSON UNIVERSITY \\ in partial fulfillment of the \\ requirements for the degree of \\ DOCTOR OF PHILOSOPHY \\ in the program of \\ Mechanical and Industrial Engineering
}

Toronto, Ontario, Canada, 2015

CDariush Firouzi, 2015 


\section{AUTHOR'S DECLARATION FOR ELECTRONIC SUBMISSION OF A DISSERTATION}

I hereby declare that I am the sole author of this dissertation. This is a true copy of the dissertation, including any required final revisions, as accepted by my examiners.

I authorize Ryerson University to lend this dissertation to other institutions or individuals for the purpose of scholarly research.

I further authorize Ryerson University to reproduce this dissertation by photocopying or by other means, in total or in part, at the request of other institutions or individuals for the purpose of scholarly research.

I understand that my dissertation may be made electronically available to the public. 


\title{
AN INVESTIGATION OF THE MECHANICAL AND BIOLOGICAL PROPERTIES OF NYLON COATED ULTRA-HIGH-MOLECULAR-WEIGHT POLYETHYLENE
}

\author{
Dariush Firouzi \\ Doctor of Philosophy, Mechanical \& Industrial Engineering, Ryerson University, Toronto (2015)
}

\begin{abstract}
In spite of considerable improvements in the manufacturing of body armor using lightweight and high-strength fibres, demands for lighter and more flexible products capable of providing sufficient protection against various types of threats has not waned. Among high strength fibres, ultra high molecular weight polyethylene (UHMWPE) possesses superior mechanical and physical properties. Nevertheless, the use of UHMWPE fabric with a shear thickening fluid (STF) materials for the manufacture advanced liquid body armors has been unsuccessful as this polymer is inherently inert and cannot bond or readily interact with other materials. To address these challenges, this research thesis focused on the development of a new method to increase the performance of UHMWPE fibre/fabric for high impact applications and improve its capability to bond with silica-based STF materials. A novel coating technique was developed using a nylon solution with UHMWPE fibre which results in a strong interlocking mechanism and the creation of a uniform coating without adding extra thickness to the fibre. Standard penetration tests showed considerable improvement in puncture/stab resistance of a nylon coated UHMWPE fabric compared with the uncoated fabric (with equivalent areal density) in terms of energy absorption, which was also explained by the scanning electron microscope (SEM) images of ruptured areas. In order to further improve the penetration resistance of UHMWPE fabric, a dispersion of fumed silica particles in polyethylene glycol (PEG) was synthesized exclusively via
\end{abstract}


a novel sequential ultrasonication technique to incorporate with a multilayer stack of UHMWPE fabric. A complete set of rheological measurements, zeta potential and SEM analysis were done to study the viscoelastic characteristics and tune the stability of the synthesized STF samples. Evidence of the improved adhesion of STF materials to UHMWPE fibres coated with nylon from the SEM images was observed. Finally, while the evidence of improved mechanical properties of nylon coated UHMWPE were provided (e.g. higher creep resistance and toughness), its potential application for the manufacturing of medical devices was also explored. As a result, from the preliminary cytotoxicity and osteolysis assessments, it was shown that the biological compatibility of UHMWPE was improved when it was coated with nylon. 


\section{ACKNOWLEDGMENTS}

I start by thanking my supervisor, Dr. Habiba Bougherara, for her continued encouragement, guidance, and support throughout the course of my PhD studies, and the completion of this dissertation would not have been possible otherwise. I am grateful to my co-supervisor, Dr. Daniel Foucher, who provided me insightful discussions and guidance in this research. Dr. Foucher also helped me to be involved with our industrial partner: Mr. Pradeep Agarwal (Electro-Pack Inc., Ontario). I am very thankful to Mr. Agarwal for his generous financial and technical support throughout my study. I am also grateful to Dr. Asma Amlesh's team at the American University in Cairo, Egypt, for helping me with the biological experiments. I am indebted to Professor Marcello Papini for providing me access to the equipment and research facilities in his laboratory. I am also indebted to Professor Derick Rousseau for giving me the opportunity to use the rheometer and several other testing instruments in his laboratory. I would like to give special thanks to Dr. Hassan Firoozmand, Dr. Ihab El Sawi, Dr. Hamid Ghaemi, Mr. Alan Machin, Mr. Muhammad Saleem, Mr. Pouria Tavakkoli-Avval, Ms. Zahra Bagheri, Mr. Saeid Samiezadeh, Ms. Maryam Abdinejad, and Mr. Muhammad Yousaf for the many good discussions about various aspects of this research and for the valuable help. Finally, I would like to acknowledge Federal Economic Development Agency for Southern Ontario (FedDev Ontario) and Scientists and Engineers in Business (SEB) Commercialization Fellowship for financially supporting this research project. 


\section{TABLE OF CONTENTS}

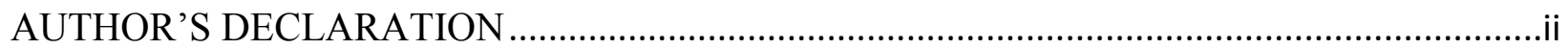

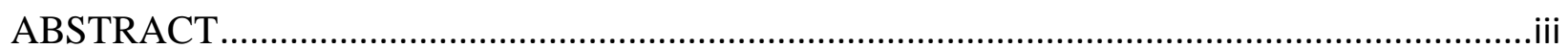

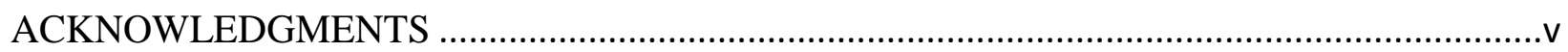

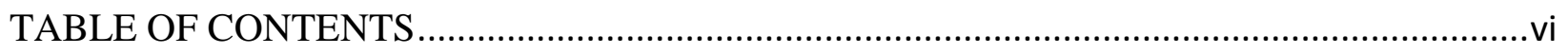

LIST OF TABLES ...........................................................................................................

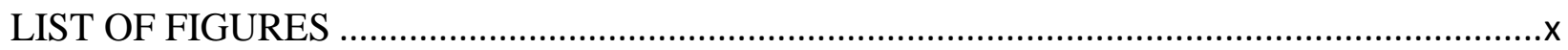

LIST OF ABBREVIATIONS ................................................................................

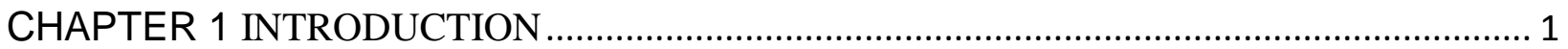

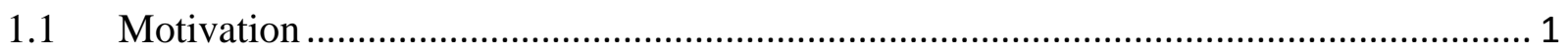

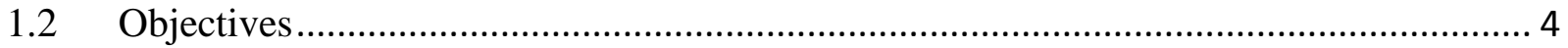

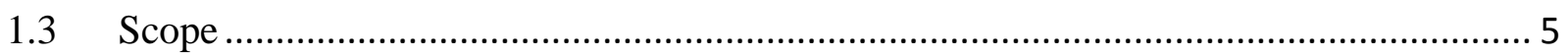

CHAPTER 2 LITERATURE SURVEY AND BACKGROUND............................................ 7

2.1 High Performance Fibre for Armor Application; Advanced Soft Body Armor .............. 7

2.1.1 Ultra High Molecular Weight Polyethylene (UHMWPE) ....................................... 9

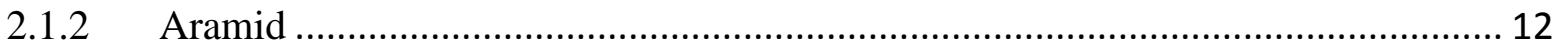

2.2 Mechanisms of Impact Energy Dissipation in Multilayer Textiles .............................. 13

2.2.1 Effect of Frictional Force ............................................................................... 13

2.2.2 Effect of Various Coating Materials ………….................................................... 15

2.2.3 Effect of Boundary Condition and Stitching ...................................................... 17

2.3 Textile/resin Composites for Advanced Protection ...................................................... 18

$2.4 \quad$ Shear Thickening Fluids (STFs) …………............................................................... 19

2.4.1 Shear Thickening Phenomenon ………............................................................. 19

2.4.2 Applications for STF Materials ............................................................................. 20

2.4.3 Textile-STF with Advanced Protective Performance ............................................ 21

2.4.4 Mechanism of Enhanced Penetration Resistance of Liquid Body Armor .............. 22

Water Absorption; A Drawback of Using STF Materials....................................... 25

Influence of STF Characteristics in Liquid Body Armor....................................... 26

2.4.5 Influence of Ultrasonication on the Viscoelastic Characteristics of STF .............. 28

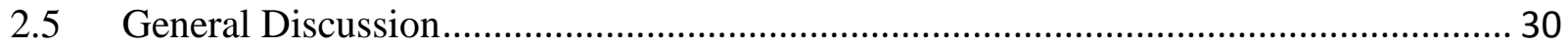


3.1 Nylon Coating: A Novel Solution for Protective Clothing ..................................... 32

3.1.1 Material Details................................................................................................ 32

3.1.2 Sample Preparation; Nylon Coated Fibre/Fabric ........................................... 33

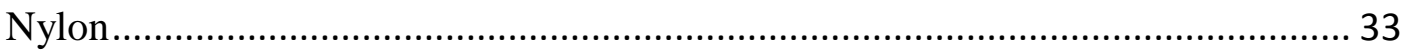

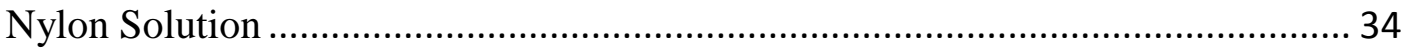

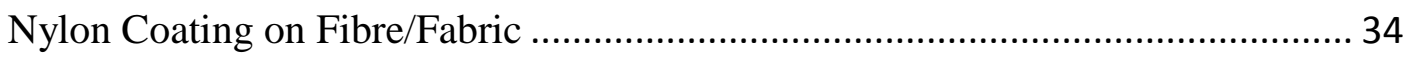

3.1.3 Preliminary Evaluation of Nylon Coating …................................................. 35

Flexibility Test and Thickness Measurement ............................................... 35

Scanning Electron Microscopy (SEM) ….................................................... 36

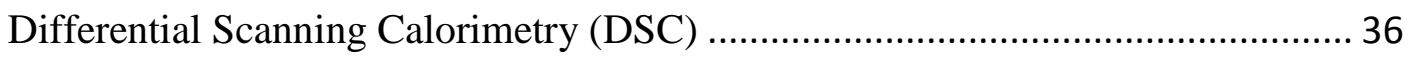

3.1.4 Dynamic Impact Penetration Test on Fabrics ............................................... 36

3.1.5 Quasi-Static Penetration Test On Fabrics ......................................................... 40

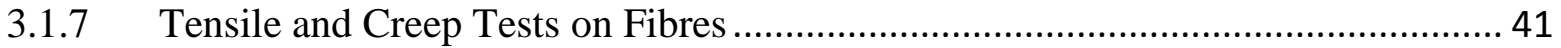

3.2 Shear Thickening Fluid (STF); A Novel Approach to Fabricate a Stable STF ............ 42

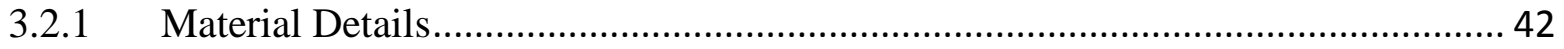

3.2.2 Sample Preparation; STF Fabrication ....................................................... 42

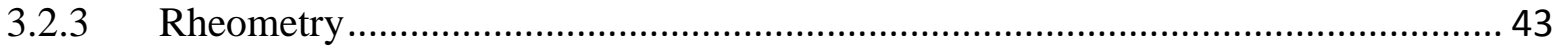

3.2.4 Zeta Potential Measurement and SEM analysis.............................................. 44

CHAPTER 4 TEST RESULTS AND DISCUSSION ......................................................... 45

4.1 Nylon Coating; Mechanical and Penetration Test Results ..................................... 45

4.1.1 Morphological Analysis and Measurements................................................. 45

4.1.2 The Impact of $\mathrm{CaCl}_{2}$ on Nylon Coating .................................................... 48

4.1.3 Dynamic Impact Penetration Test Results on Fabrics ...................................... 50

4.1.4 Quasi-static Penetration Test Results on Fabrics............................................ 51

Fabric Damage Mechanisms in Quasi-static Penetration Test............................ 56

4.1.5 Tensile/Creep Test Results on Fibres ........................................................... 58

Fibre Damage Mechanisms in Tension/Creep ............................................... 64

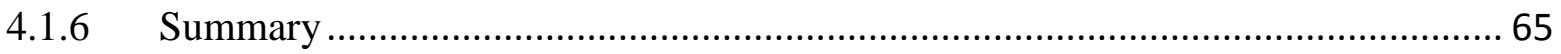

4.2 STFs; Rheological Measurements and Morphological Analysis ............................. 66 


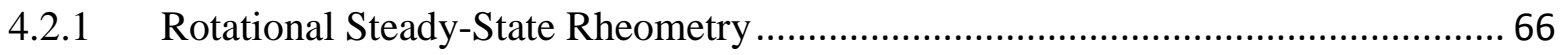

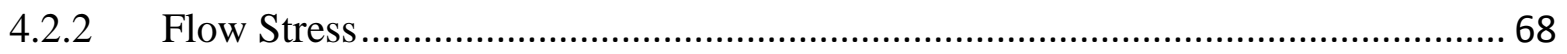

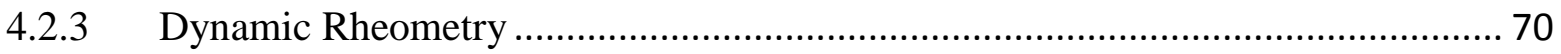

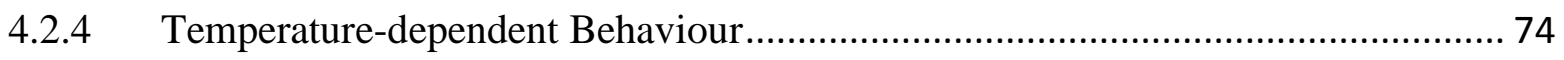

4.2.5 Zeta potential measurement and SEM analysis .................................................... 76

4.2.6 Discussion on the Rheological Properties of Fumed Silica Particles ..................... 78

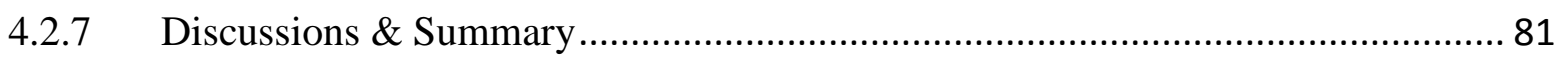
The Effectiveness of Nylon Coating on UHMWPE Fabric to Host STF Materials 82 CHAPTER 5 BIOLOGICAL APPROACH; A POTENTIAL APPLICATION ……….............. 85

5.1 Introduction; Biological Aspects of UHMWPE Material .............................................. 85

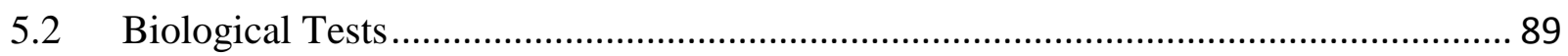

5.2.1 Preparation of Neat and Nylon 6,6 Coated UHMWPE Extracts ........................... 89

5.2.2 Cell Culture for L929 Fibroblasts and U-937 Human Macrophage Cell Lines...... 89

5.2.4 Investigation of Osteolysis by RT-PCR ............................................................ 92

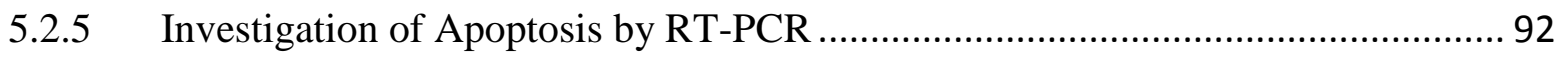

5.2.6 Statistical Analysis and Statistical Comparison................................................... 92

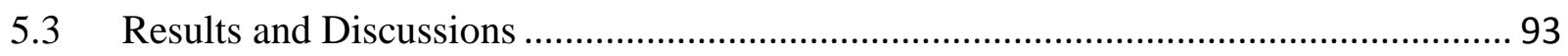

5.3.1 MTT Results .................................................................................................... 93

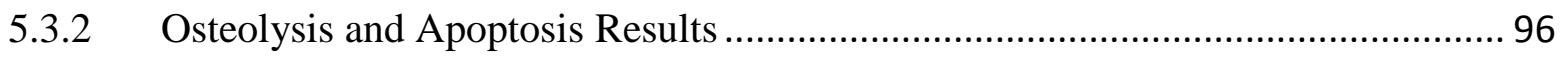

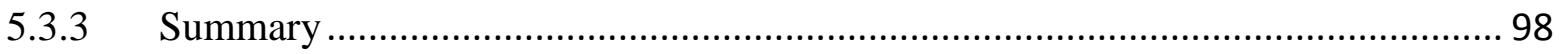

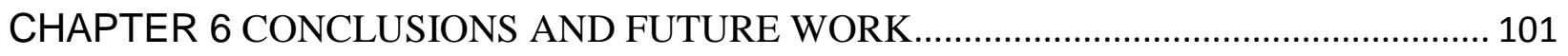

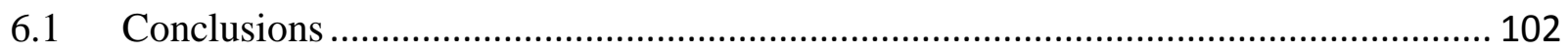

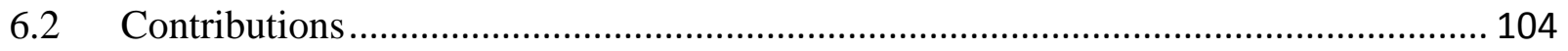

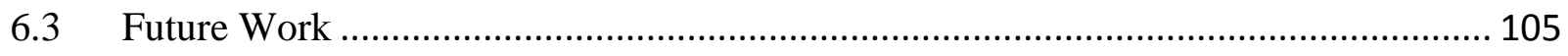

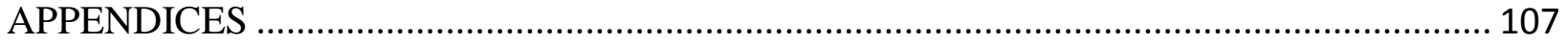

A.1 Apoptosis Assay Results ..................................................................................... 107

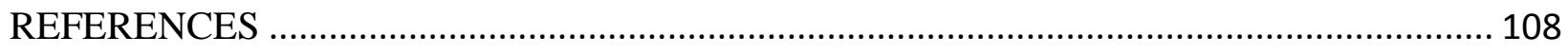




\section{LIST OF TABLES}

Table 1. Various mechanical properties of aramid, UHMWPE, S-glass, and PBO fibres, Adopted from [40].

Table 2. Typical average physical and mechanical properties of bulk high-density polyethylene (HDPE) and bulk ultra-high-molecular-weight polyethylene (UHMWPE), Adopted from [43]..........................10

Table 3. Relative depth of penetration in dynamic impact test on fabrics. ............................................ 38

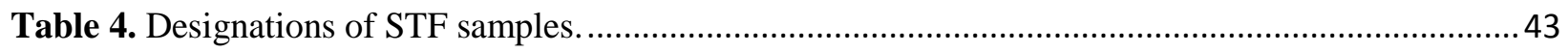

Table 5. Flexibility test results on neat and nylon coated UHMWPE fabric. ......................................... 47

Table 6. Dynamic impact penetration test results on fabrics for different weapons at two impact energies.

Table 7. Energy dissipation in quasi-static penetration test for different weapons on a 2 or 3 layer stack of fabric. 55

Table 8. Energy dissipation in quasi-static penetration test for different weapons on a single layer fabric.

Table 9. Tensile properties of neat and nylon coated UHMWPE fibres at 25, 50, and $70{ }^{\circ} \mathrm{C} \ldots \ldots \ldots \ldots \ldots . . . . . . . .61$

Table 10. Maximum creep time of neat and nylon coated UHMWPE fibres at 25 and $50^{\circ} \mathrm{C} \ldots . . . . . . . . . . . . . . .63$

Table 11. Maximum creep time of neat and nylon coated UHMWPE fibres at $70{ }^{\circ} \mathrm{C}$..........................6 63

Table 12. Critical shear rate and maximum shear thickened viscosity for steady-shear stress controlled rheometry. 68

Table 13. Temperatures at the crossover point of $\mathrm{G}^{\prime}$ and $\mathrm{G}^{\prime \prime}$ moduli for STF samples (temperature sweep oscillation at $\gamma=1 \%$ and $\omega=1 \mathrm{rad} / \mathrm{s}$ with the heating and cooling rate of $3{ }^{\circ} \mathrm{C} / \mathrm{min}$ for three cycles)..... 76 


\section{LIST OF FIGURES}

Figure 1. The percentage of the total victims of violent crimes by different weapons from 1999 to 2008 in Canada [6] 1

Figure 2. A comparison of the tensile properties of various high strength fibres with a steel wire [14]. .... 3

Figure 3. Schematics of the various structures of woven fabrics [40]. .

Figure 4. A TEM image of UHMWPE showing amorphous (gray color) and crystalline regions (white lines with a dark out-line) [43]....

Figure 5. A schematic of the morphological features of UHMWPE [43] .............................................. 11

Figure 6. Aramid chemical structures [10] ............................................................................... 12

Figure 7. A schematic of the change in the microstructure of a colloidal dispersion with a shear rate(the

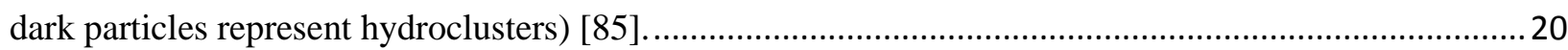

Figure 8. Chemical reaction to synthesize Nylon 6,6, Adopted from [115]. ............................................ 33

Figure 9. Structure of Nylon 6,6, Adopted from [114] ................................................................... 34

Figure 10. A schematic of the flexibility test setup for fabrics.......................................................... 36

Figure 11. A schematic of the witness-papers placed within a backing material pack in dynamic

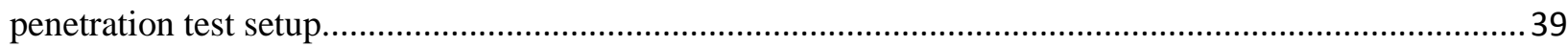

Figure 12. Weapons used for the penetration test on fabrics according to the NIJ Standard 0115.00 [116]:

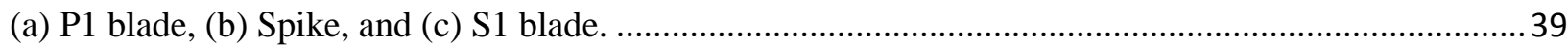

Figure 13. Drop mass weapon used for dynamic impact test on fabric samples according to the NIJ

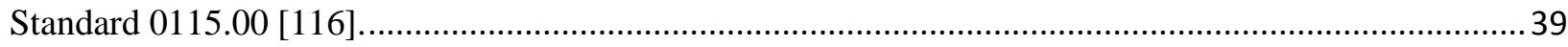

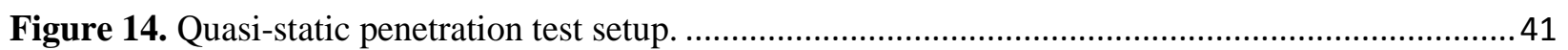

Figure 15. Tensile and creep test setup for fibres. ................................................................... 42

Figure 16. Cross section area of nylon coated UHMWPE fibre at different magnification (a) $\times 140$ and (b)

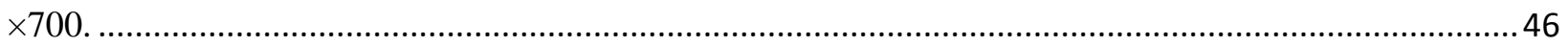

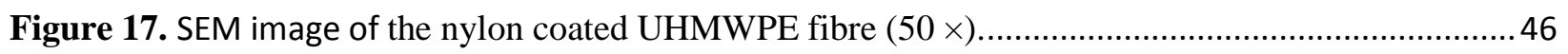

Figure 18. A magnified ( $\times 25)$ top view of fabrics: (a) Neat UHMWPE, (b) Nylon 6,6 coated UHMWPE,

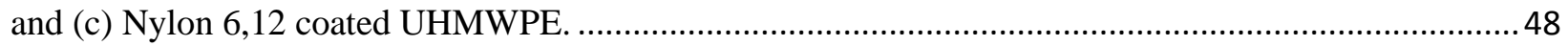

Figure 19. Chemical analysis of nylon dissolution, Adopted from [120]........................................ 49

Figure 20. DSC graphs: —

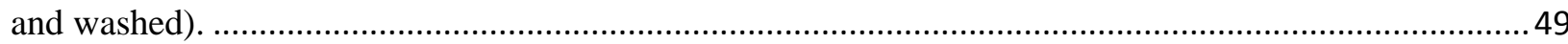

Figure 21. Cross-section view: (a) neat UHMWPE fabric, (b) Nylon 6,6 coated UHMWPE fabric, and (c)

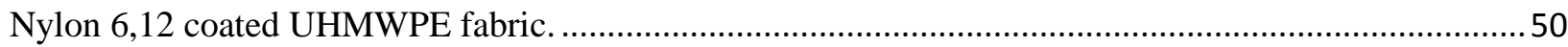

Figure 22. Force versus displacement graphs from the quasi-static penetration test results with a spike on: — 3 layer stack of neat UHMWPE fabric; --------, 2 layer stack of Nylon 6,6 coated UHMWPE fabric; ......., 2 layer stack of Nylon 6,12 coated UHMWPE fabric......

Figure 23. Force versus displacement graphs from the quasi-static penetration test results with an S1 blade on: — 3 layer stack of neat UHMWPE fabric; --------, 2 layer stack of Nylon 6,6 coated UHMWPE fabric;........, 2 layer stack of Nylon 6,12 coated UHMWPE fabric...................................53

Figure 24. Force versus displacement graphs from the quasi-static penetration test results with aP1 blade on: — 3 layer stack of neat UHMWPE fabric; ---------, 2 layer stack of Nylon 6,6 coated UHMWPE fabric; ........, 2 layer stack of Nylon 6,12 coated UHMWPE fabric. .54 
Figure 25. SEM images of the ruptured area after quasi-static penetration test on neat UHMWPE fabric using: (a) Spike; (b) S1 blade; (c) P1 blade.

Figure 26. SEM images of the ruptured area after quasi-static penetration test on Nylon 6,12 coated UHMWPE fabric using: (a) Spike; (b) S1 blade; (c) P1 blade.

Figure 27.SEM images of the ruptured area after quasi-static penetration test on Nylon 6,12 coated UHMWPE fabric using: (a) Spike; (b) S1 blade; (c) P1 blade.

Figure 28. Force versus displacement graphs from the tensile test at $25^{\circ} \mathrm{C}$ : - , neat UHMWPE fibre; --------, Nylon 6,6 coated UHMWPE fibre; ........, Nylon 6,12 coated UHMWPE fibre (gauge length of $250 \mathrm{~mm}$ and cross speed of $2.5 \mathrm{~mm} / \mathrm{s}$ ).

Figure 29. Force versus displacement graphs from the tensile test at $50{ }^{\circ} \mathrm{C}:-\longrightarrow$, neat UHMWPE fibre; --------, Nylon 6,6 coated UHMWPE fibre; ........, Nylon 6,12 coated UHMWPE fibre (gauge length of $250 \mathrm{~mm}$ and cross speed of $2.5 \mathrm{~mm} / \mathrm{s}$ ).

Figure 30. Force versus displacement graphs from the tensile test at $70^{\circ} \mathrm{C}:-$, neat UHMWPE fibre; --------, Nylon 6,6 coated UHMWPE fibre; ........, Nylon 6,12 coated UHMWPE fibre (gauge length of $250 \mathrm{~mm}$ and cross speed of $2.5 \mathrm{~mm} / \mathrm{s}$ ).

Figure 31. Strain versus time graphs from the creep test at a fixed load of $190 \mathrm{~N}$ at $25{ }^{\circ} \mathrm{C}$ : ——, neat UHMWPE fibre; --------, Nylon 6,6 coated UHMWPE fibre; ........, Nylon 6,12 coated UHMWPE fibre (gauge length of $250 \mathrm{~mm}$ ).

Figure 32. Strain versus time graphs from the creep test at a fixed load of $190 \mathrm{~N}$ at $50{ }^{\circ} \mathrm{C}$ : — , neat UHMWPE fibre; ---------, Nylon 6,6 coated UHMWPE fibre; ........, Nylon 6,12 coated UHMWPE fibre (gauge length of $250 \mathrm{~mm}$ ).

Figure 33. Strain versus time graphs from the creep test at a fixed load of $120 \mathrm{~N}$ at $70{ }^{\circ} \mathrm{C}$ : — , neat UHMWPE fibre; ---------, Nylon 6,6 coated UHMWPE fibre; ........, Nylon 6,12 coated UHMWPE fibre (gauge length of $250 \mathrm{~mm}$ ).

Figure 34. (a) SEM image of the ruptured area of a Nylon 6,6 coated UHMWPE fibre from tension at 25 ${ }^{\circ} \mathrm{C}(20 \times)$; (b) SEM image of a Nylon 6,6 coated UHMWPE fibre prior to the progressive breakage of filaments in tension at $25^{\circ} \mathrm{C}(60 \times)$.

Figure 35. SEM image of the ruptured area of a Nylon 6,6 coated UHMWPE fibre from creep test at 25 ${ }^{\circ} \mathrm{C}(15 \times)$.

Figure 36. Apparent viscosity as a function of shear rate at different times of ultrasonication (at the last stage of fabrication process) and weight fractions of fumed silica particles in PEG (steady-state shear stress controlled): $\mathbf{\square}$, (STF-A), 20\% w/w, $45 \mathrm{~min}$;, (STF-B), 20\% w/w, $45 \mathrm{~min} ; \boldsymbol{\Delta}$, (STF-C), 20\% w/w, $135 \mathrm{~min}$; $\star$, (STF-C*), 20\% w/w, $450 \mathrm{~min}$; $\square$, (STF-D), 23\% w/w, $45 \mathrm{~min}$; O, (STF-E), 23\% w/w, $45 \mathrm{~min} ; \triangle$, (STF-F), 23\% w/w, $135 \mathrm{~min}$.

Figure 37. Storage and loss modulus as a function of shear stress (and $\omega=1 \mathrm{rad} / \mathrm{s}$ ) at different times of ultrasonication (at the last stage of fabrication process) and the same concentration of fumed silica particles in PEG (20\% w/w): $\mathbf{\square}, \square$, (STF-A), $45 \mathrm{~min} ; \boldsymbol{\bullet}, \bigcirc$, (STF-B), $45 \mathrm{~min} ; \boldsymbol{\Delta}, \triangle,(\mathrm{STF}-\mathrm{C}), 135$ min; filled symbols: G'; empty symbols: G',

Figure 38. Storage and loss modulus as a function of shear stress (and $\omega=1 \mathrm{rad} / \mathrm{s}$ ) at different times of ultrasonication (at the last stage of fabrication process) and the same concentration of fumed silica particles in PEG (23\% w/w): $\mathbf{\square}, \square$, (STF-D), $45 \mathrm{~min}$;, , O, (STF-E), $45 \mathrm{~min} ; \boldsymbol{\Delta}, \Delta$, (STF-F), $135 \mathrm{~min}$; filled symbols: G'; empty symbols: G'. 
Figure 39. Storage and loss modulus as a function of strain amplitude (and $\omega=1 \mathrm{rad} / \mathrm{s}$ ) at different times of ultrasonication (at the last stage of fabrication process) and the same concentration of fumed silica particles in PEG (20\% w/w): $\mathbf{\square}, \square$, (STF-A), $45 \mathrm{~min}$; $\bullet$, O, (STF-B), $45 \mathrm{~min} ; \boldsymbol{\Delta}, \triangle$, (STF-C), 135 min; filled symbols: G'; empty symbols: G".

Figure 40. Storage and loss modulus as a function of strain amplitude (and $\omega=1 \mathrm{rad} / \mathrm{s}$ ) at different times of ultrasonication (at the last stage of fabrication process) and the same concentration of fumed silica particles in PEG (23\% w/w): $\mathbf{\square}, \square$, (STF-D), $45 \mathrm{~min}$;, , O, (STF-E), $45 \mathrm{~min} ; \boldsymbol{\Lambda}, \triangle$, (STF-F), $135 \mathrm{~min}$; filled symbols: G'; empty symbols: G".

Figure 41. Storage and loss modulus as a function of angular frequency $(\gamma=1 \%)$ at different times of ultrasonication (at the last stage of fabrication process) and the same concentration of fumed silica particles in PEG (20\% w/w): $\mathbf{\square}, \square$, (STF-A), $45 \mathrm{~min}$; $\bullet$, O, (STF-B), $45 \mathrm{~min}$; $\mathbf{\Delta}, \triangle$, (STF-C), 135 min; filled symbols: G'; empty symbols: G".

Figure 42. Storage and loss modulus as a function of angular frequency $(\gamma=1 \%)$ at different times of ultrasonication (at the last stage of fabrication process) and the same concentration of fumed silica particles in PEG (23\% w/w): $\mathbf{\square}, \square$, (STF-D) 23\%, $45 \mathrm{~min}$; $\boldsymbol{\bullet}$, O, (STF-E) 23\%, $45 \mathrm{~min} ; \boldsymbol{\Delta}, \Delta,(\mathrm{STF}-\mathrm{F})$ 23\%, 135 min; filled symbols: G'; empty symbols: G".

Figure 43. Storage and loss modulus as a function of temperature $(\gamma=1 \%, \omega=1 \mathrm{rad} / \mathrm{s})$ at different times of ultrasonication (at the last stage of fabrication process) and the same concentration of fumed silica particles in PEG (20\% w/w): $\mathbf{\square}, \square$, (STF-A), $45 \mathrm{~min} ; \boldsymbol{\bullet}, \mathrm{O}$, (STF-B), $45 \mathrm{~min}$; $\boldsymbol{\Delta}, \triangle$, (STF-C), 135 min; filled symbols: G'; empty symbols: G"; dash line: temperature.

Figure 44. Storage and loss modulus as a function of temperature $(\gamma=1 \%, \omega=1 \mathrm{rad} / \mathrm{s})$ at different times of ultrasonication (at the last stage of fabrication process) and the same concentration of fumed silica particles in PEG (23\% w/w): $\mathbf{\square}, \square$, (STF-D), $45 \mathrm{~min}$; $\bullet$, , (STF-E), $45 \mathrm{~min} ; \boldsymbol{\Lambda}, \triangle$, (STF-F), $135 \mathrm{~min}$; filled symbols: G'; empty symbols: G"; dash line: temperature.

Figure 45. SEM images of fumed silica agglomerates after PEG medium was removed by heating from the dispersion, at different times of ultrasonication (at the latest stage of fabrication process) and different weight fractions of fumed silica particles: a) as-received fumed silica; b) (STF-D), 23\% w/w, $45 \mathrm{~min}$; c) (STF-A), 20\% w/w, $45 \mathrm{~min}$; d) (STF-B), 20\% w/w, $90 \mathrm{~min}$; e) (STF-C), 20\% w/w, $135 \mathrm{~min}$; f) (STF-C*), $20 \% \mathrm{w} / \mathrm{w}, 450 \mathrm{~min}$.

Figure 46. SEM images: (a) STF impregnated neat UHMWPE fabric, (b) STF impregnated Nylon 6,6 coated UHMWPE fabric (the STF material is comprised of $23 \% \mathrm{w} / \mathrm{w}$ amorphous fumed silica dispersion in PEG-200).

Figure 47. Effect of neat and nylon coated UHMWPE extracts on the viability of L929 fibroblast cells at 24, 48, and $72 \mathrm{~h}(* \mathrm{p}<0.05$, ** $\mathrm{p}<0.01$, and ***p < 0.001): $\square$, Cell+fresh medium; $\square$, Cells+shaked medium;, , Neat UHMWPE; $\mathbf{\square}$, Nylon 6,6 coated UHMWPE. 95

Figure 48. Magnified $(\times 200)$ microscopic images of the cell viability of L929 fibroblasts upon exposure to three conditions, i.e. control (normal fresh medium+cells), neat UHMWPE, and Nylon 6,6 coated UHMWPE, at three incubation periods (24, 48, and $72 \mathrm{~h}$ ). .96

Figure 49. Expression levels of TNF $\alpha$ marker at 24, 48, and 72 incubation periods ( ${ }^{*} \mathrm{p}<0.05$, ** p < 0.01, and $* * * p<0.001): \mathbf{\square}$, Cell+fresh medium; , Neat UHMWPE; $\square$, Nylon 6,6 coated UHMWPE. 
Figure 50. Expression levels of IL-6 marker at 24, 48, and $72 \mathrm{~h}$ incubation periods $(* \mathrm{p}<0.05$, **p < 0.01, and $* * * p<0.001): \mathbf{\square}$, Cell+fresh medium; , Neat UHMWPE; $\square$, Nylon 6,6 coated UHMWPE.

Figure 51. Expression levels of Bax marker at 24, 48, and $72 \mathrm{~h}$ incubation periods: $\mathbf{\square}$, Cell+fresh medium; - , Neat UHMWPE; $\square$, Nylon 6,6 coated UHMWPE....................................................107

Figure 52. Expression levels of Caspase 3 marker at 24, 48, and 72 h incubation periods: $\mathbf{\square}$, Cell+fresh medium; . , Neat UHMWPE; $\square$, Nylon 6,6 coated UHMWPE..................................................... 107 


\section{LIST OF ABBREVIATIONS}

Analysis of Variance (ANOVA)

Carbon Nanotubes (CNTs)

Differential Scanning Calorimetry (DSC)

Dulbecco's Modified Eagle Medium (DMEM)

Fetal Bovine Serum (FBS)

hexamethylenediamine (HMD)

High density polyethylene (HDPE)

Hydroxyapatite (HA)

Interleukin 6 (IL-6)

Linear Low Density Polyethylene (LLDPE)

Low Density Polyethylene (LDPE)

Methyl ThiazolTetrazolium (MTT)

Multi-Wall Carbon Nanotubes (MWCNTs)

Nano-Size Chain Aggregates (NCA)

Natural Rubber Latex (NRL)

Order-Disorder Transition (ODT)

Phosphate Buffered Saline (PBS)

Polyethylene (PE)

Polyethylene Glycol (PEG)

Polymer Matrix Composite (PMC)

Real-time Polymerase Chain Reaction (RT-PCR)

Polymethyl Methacrylate (PMMA)

Polyphenylene Benzobisoxazole (PBO)

Polyvinyl Butyral (PVB)

Roswell Park Memorial Institute (RPMI)

Scanning Electron Microscopy (SEM)

Shear Thickening Fluid (STF)

Total Hip Arthroplasty (THA)

Transmission Electron Microscopy (TEM)

Transmitted Transverse Stress Wave (TSW)

Tumor Necrosis Factor Alpha (TNFa)

Ultra High Molecular Weight Polyethylene (UHMWPE)

Unidirectional (UD) 


\section{CHAPTER 1 INTRODUCTION}

\subsection{Motivation}

Body armor has traditionally been designed to provide a protection against ballistic threats. Today, in real life situations, because of the increasing number of stab and puncture threats committed with knives and sharpened instruments, stab resistance is becoming increasingly more important. There are several applications which demand advanced puncture/stab resistance, such as the required protection for correctional officers. For military applications, stab resistant armor is highly desirable in close-quarters combat situations [1-5].

In 2008, $18 \%$ of all crimes in Canada were committed with a weapon. Knives are the most common threat $(6 \%)$ when a weapon is used to commit a violent crime. Broken bottles, screwdrivers, scissors or other piercing and cutting instruments are also considered as a knife attack. In comparison, only $2 \%$ of all violent crimes were committed with a firearm and $3 \%$ with a club or blunt instrument in 2008. In just 2008, it was reported that a knife attack was involved in about 23,500 of violent crimes in Canada [6]. Figure 1 shows the rate of violent crimes with knives from 1999 to 2008 in Canada.

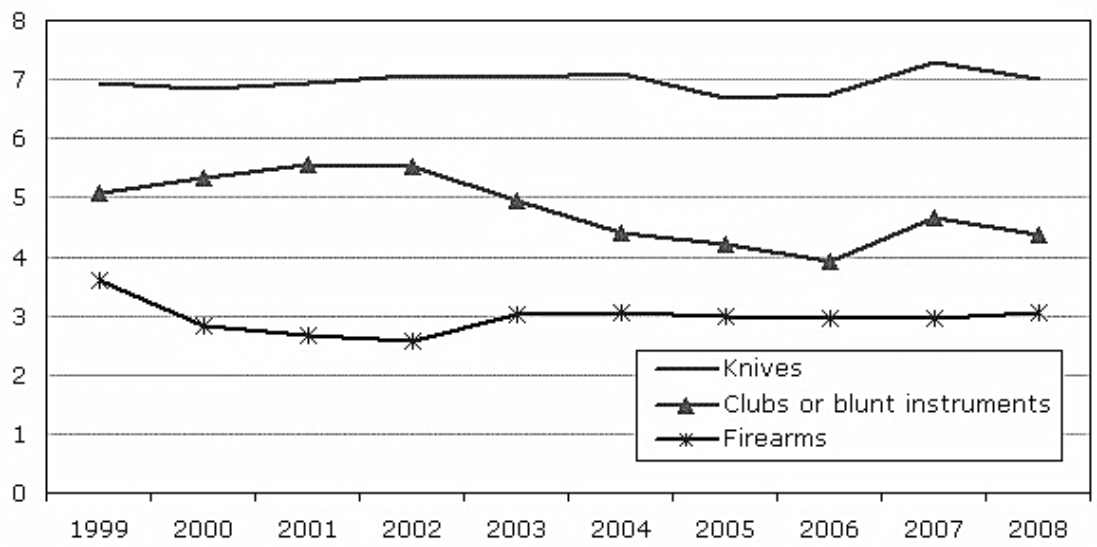

Figure 1. The percentage of the total victims of violent crimes by different weapons from 1999 to 2008 in Canada [6]. 
Generally, it is easier to stop a bullet than a spike or stab threat because the impact speed of bullets is hundreds of metres per second versus a few metres per second for a knife attack. Also, a bullet normally weighs about a few grams while the combination of a knife and a person's arm weighs several kilograms. Hence, the bullet can flatten on impact, which creates a large impact surface area while the shape of the knife blade does not change during penetration [4]. Stab threats can be classified into two categories: (i) Puncture attacks and (ii) Cut attacks. Puncture is when an instrument's sharp pointed tip penetrates into a target without leaving a cutting edge, such as the puncture impact of ice picks or awls. This kind of threat is of the primary concern to correctional officers, since a sharply-pointed object can be fabricated relatively easy in penitentiaries. A cut threat refers to the impact of knife-like objects with cutting edges. Such a threat is more difficult to stop rather than a puncture because of the shape of the blade-type penetrator with continuous sharp cutting edges [1-3,5]. More specifically, a stab threat is when the direction of a knife or stabbing weapon is perpendicular to the target. In contrast, a slash threat is when the weapon is swept in a parallel direction to the target [5].

A new class of advanced body armor was created after the development of high-strength synthetic fibres. This kind of body armor can provide superior ballistic performance compared to traditional ones such as chain mail and hard body armor with titanium or metallic sheets. The conventional body armor was bulky, inflexible, and not easily concealable; although it could provide a high level of resistance against ballistic or stab threats [1,2,7-9]. High performance fibres, such as polyamides, aramid, S-glass, ultra high molecular weight polyethylene (UHMWPE) or polyphenylene benzobisoxazole (PBO), generally possess high strength, low density, high compressive strength, high energy absorption, and good adhesion to matrices for composite production $[8,10,11]$. A tightly woven fabric from a high strength fibre absorbs a 
significantly higher kinetic energy of the ballistic impact per its body weight than metal alloys, ceramics or composites [12,13]. In Figure 2, the tensile properties of various high strength fibres were compared to a steel wire.

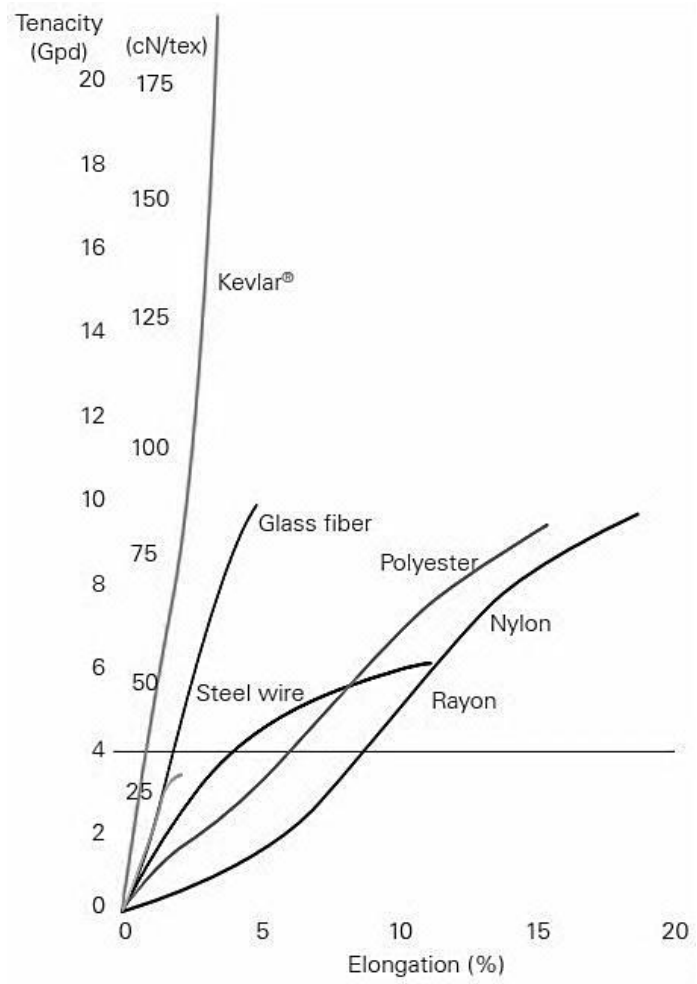

Figure 2. A comparison of the tensile properties of various high strength fibres with a steel wire [14].

Nevertheless, this kind of soft body armor cannot provide sufficient protection against various stab threats [1]. Several commercial products are now available in market, especially for puncture/stab protection, such as $\operatorname{Kevlar}^{\circledR}$ Correctional which is made of a high strength $\operatorname{Kevlar}^{\circledR}$ fabric with a high yarn count [1,5]. Another example is a Twaron ${ }^{\odot}$ Stab Resistant Material (SRM) which is made of Twaron $^{\odot}$ fabric embedded within a silicon carbide/polymer matrix composite [7]. The drawback of these products is that they are expensive and not necessarily capable of providing sufficient ballistic protection [1,5]. Another problem of using a soft body armor made of a high strength fibre is that such a product is relatively heavy and stiff because 
several dozen stacked fabrics are required to prevent lethal injuries against multi-threats [9$11,15-18]$

Liquid body armor is a novel composite technology which is composed of a non-Newtonian shear thickening fluid (STF) material impregnated into a high performance fabric. The incorporation of the STF material onto fabrics to enhance the armor's ballistic resistance has attracted much interest in the past decade. The key advantage of this novel technology is that the high viscosity STF material immediately transits into a solid-like state under an impact force, making the permeated fabric stronger. The STF material then reverts to its original viscous-like state again shortly afterwards and remains flexible during a normal wear [1,2,9,10,16-38]. Providing a similar range of penetration resistance as textile-only body armor, the STF-fabric body armor is even thinner and more flexible with the same areal density and requires fewer number of fabric layers [1,2,10,37-39]. Such a liquid body armor has the potential to provide an advanced multi-threat protection against both ballistic and stab threats [2]. Despite considerable improvement in the manufacturing of body armor, there is an everlasting demand to make lighter body armor with better performance and greater flexibility.

\subsection{Objectives}

The main purpose of this study is to develop a new composite material, based on UHMWPE, to overcome the limitations of current security applications including failure to stop punctures and stab threats, high cost, and bulky weight. UHMWPE has the lowest density and highest specific strength among high performance fibres. In addition, UHMWPE fibre is relatively cheap and has a negligible moisture absorption (less than $0.01 \%$ ). Despite the superior mechanical properties of UHMWPE, there are considerably few research studies regarding the use of UHMWPE fibre/fabric for liquid body armor application compared to other high strength fibres, such as 
Kevlar $^{\circledR}$. The main objective of this research was broken down into three sub-objectives: (1) To improve the interfacial properties of UHMWPE fibre's surface, (2) To incorporate high performance and stable STF materials into a multilayer stack of the treated UHMWPE fabric, and (3) To evaluate the mechanical performance and penetration resistance of new composite materials developed in this research.

\subsection{Scope}

A comprehensive literature survey of the evolution of body armor, mechanisms of impact dissipation in multilayer textiles and textile-STF systems, rheological behaviour of STF materials and viscoelastic characteristics of various fumed silica dispersions, and incorporation of STF materials with - textiles for body armor application is presented in Chapter 2.

A new technique which was developed in our research group, to improve the mechanical performance and penetration resistance of UHMWPE fibre/fabric, will be presented in Chapter 3. As the surface of UHMWPE material is inherently inert, it cannot bond or readily interact with other materials, including STFs. Chapter 3 contains an overview of a new technique to alter the mechanical characteristics of UHMWPE using a nylon coating: (i) A detailed procedure of making a suitable nylon solution, (ii) An explanation about the procedure of nylon coating using a hand-made automatic coating machine, (iii) Mechanical test procedures to evaluate the tensile and creep performance of nylon coated UHMWPE fibre, (iv) Penetration test procedures to evaluate the performance of nylon coated UHMWPE fabric against a knife threat and complementary morphological analysis. A new approach to fabricate a stable and high performance STF material via a sequential ultrasonication method will also be explained in Chapter 3. Although the shear thickening behavior of various colloidal dispersions has previously been investigated for different rheological aspects in a number of research studies, 
few studies have been conducted to study the stability of such a material, and even fewer studies have been conducted to examine the viscoelastic characteristics of fumed silica colloidal dispersion. The detailed test results and discussion for all the mechanical and rheological experiments and analysis will be presented in Chapter 4.

A new potential use of the nylon coated UHMWPE material will be presented in Chapter 5, which is focussed primarily on clinical applications. Chapter 5 includes a comprehensive literature survey of the biological aspects of UHMWPE composite materials, biological test procedures to evaluate the cytotoxicity and osteolysis induced by the test materials, detailed biological test results and lastly a discussion of these results. In Chapter 6, a list of the contributions and concluding remarks of this study will be presented with several suggestions for future work. 


\section{CHAPTER 2 LITERATURE SURVEY AND BACKGROUND}

This chapter presents an in depth literature survey regarding various aspects relevant to this study. First, a review of the development of high strength fibres, and more specifically UHMWPE fibre, will be introduced, followed by a literature survey of the mechanisms of damage failures in protective products. Additional review of the mechanisms of energy dissipation by multilayer textile structures against different types of threats, such as knife weapon and firearm, will also be presented. A literature review of the influence of coating on the performance of multilayer textile products will be presented, followed by a literature survey of the effect of water absorption and boundary conditions. Then, a subsequent review of polymer matrix composites (PMC) reinforced with woven or non-woven high strength fibres will be presented. Next, a critical literature review of the development of liquid body armor will be provided, starting with a review of the mechanism of the shear thickening phenomenon, followed by a review of the applications of shear thickening fluid (STF) materials. Finally, a literature survey of the mechanisms of the energy dissipation by textile-STF composites will be discussed, and the influence of STF characteristics on the performance of liquid body armor will also be reviewed in more details.

\subsection{High Performance Fibre for Armor Application; Advanced Soft Body Armor}

Throughout history, it has always been a high priority to find advanced materials which are lightweight, flexible, and can protect against specific threats. Since the Korean War (1950), metal-based armors were eliminated and replaced with high strength fibres in body armor. High strength fibres can be weaved together to construct various structures, such as plain weave, basket weave, triaxial weave, 3D braid, 3D orthogonal weave, and 3D triaxial weave, each of which can provide a different level of flexibility and performance (Figure 3) [40]. In general, 
high performance fibres mainly have a high degree of molecular orientation along the fibre axis, possessing a very high molecular weight and high degree of crystallinity [41]. Various mechanical properties of different high strength fibres used in body armor are listed in Table 1.

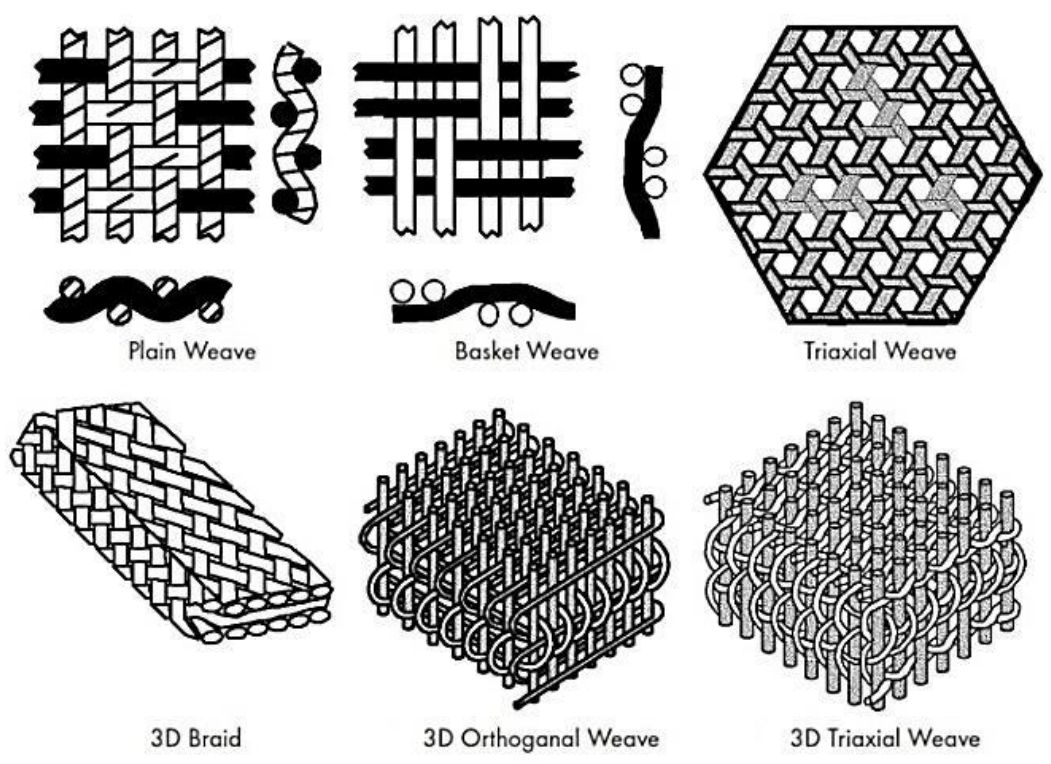

Figure 3. Schematics of the various structures of woven fabrics [40].

Table 1. Various mechanical properties of aramid, UHMWPE, S-glass, and PBO fibres, Adopted from [40].

\begin{tabular}{lccccc}
\hline Fibre & $\begin{array}{c}\text { Density } \\
\left(\mathbf{g} / \mathbf{c m}^{\mathbf{3}}\right)\end{array}$ & $\begin{array}{c}\text { Elastic } \\
\text { Modulus } \\
(\mathbf{G P a})\end{array}$ & $\begin{array}{c}\text { Tensile } \\
\text { Strength (MPa) }\end{array}$ & $\begin{array}{c}\text { Strain to } \\
\text { Failure (\%) }\end{array}$ \\
\hline Aramid & S-glass & 2.48 & 90 & 4400 & 5.7 \\
& Technora & 1.39 & 70 & 3000 & 4.4 \\
& Twaron & 1.45 & 121 & 3100 & 2.0 \\
& Kevlar-29 & 1.44 & 70 & 2965 & 4.2 \\
& Kevlar-129 & 1.44 & 96 & 3390 & 3.5 \\
& Kevlar-49 & 1.44 & 113 & 2965 & 2.6 \\
& Kevlar-KM2 & 1.44 & 70 & 3300 & 4.0 \\
& & & & & \\
& Spectra-900 & 0.97 & 73 & 2400 & 2.8 \\
& Spectra-1000 & 0.97 & 103 & 2830 & 2.8 \\
& Spectra-2000 & 0.97 & 124 & 3340 & 3.0 \\
& Dyneema & 0.97 & 87 & 2600 & 3.5 \\
& & & & & 3.5 \\
& Zylon-AS & 1.54 & 180 & 5800 & 2.5 \\
\hline
\end{tabular}


*The data presented are typical values and thus will vary dependent upon fibre denier.

High performance fibres can be used for various applications, such as making protective barriers against typical turbine engine-burst fragments in aircrafts [42].

\subsubsection{Ultra High Molecular Weight Polyethylene (UHMWPE)}

Ultra high molecular weight polyethylene (UHMWPE) has some outstanding properties, such as chemical resistance against most chemicals, excellent lubricity, high abrasion resistance, high impact resistance, and high strength. These properties made UHMWPE polymer a suitable choice for several industrial applications, such as load bearing devices. This polymer is being used in more than $90 \%$ of the total hip replacements manufactured worldwide. UHMWPE fibre has the lowest density of all high strength fibres, and its specific density is as low as 0.97 . UHMWPE is a long, linear, homopolymer comprised of ethylene $\left(\mathrm{C}_{2} \mathrm{H}_{4}\right)$ repeat units and it can also be copolymerized with other monomers such as propylene. This copolymerization can be up to $50 \%$ of the polymer and is still referred to "UHMWPE". UHMWPE molecular chain consists of more than 200,000 ethylene repeat units, which brings more than 400,000 carbon atoms in its chain's backbone with an average molecular weight of about 6 million g/mol. High density polyethylene (HDPE), linear low density polyethylene (LLDPE), and low density polyethylene (LDPE) are other kinds of this polyethylene homopolymer with different molecular weights and chain architectures [43].

Typical average physical and mechanical properties of HDPE and UHMWPE are summarized in Table 2, showing that UHMWPE has a higher ultimate tensile strength and impact strength than HDPE. 
Table 2. Typical average physical and mechanical properties of bulk high-density polyethylene (HDPE) and bulk ultra-high-molecular-weight polyethylene (UHMWPE), Adopted from [43].

\begin{tabular}{|c|c|c|}
\hline Property & HDPE & UHMWPE \\
\hline Molecular Weight $\left(1^{6} \mathrm{~g} / \mathrm{mol}\right)$ & $0.05-0.25$ & $3.5-7.5$ \\
\hline Melting Temperature $\left({ }^{\circ} \mathrm{C}\right)$ & $130-137$ & $132-138$ \\
\hline Poisson's Ratio & 0.40 & 0.46 \\
\hline Specific Gravity & $0.952-0.965$ & $0.925-0.945$ \\
\hline Tensile Modulus of Elasticity* (GPa) & $0.4-4.0$ & $0.5-0.8$ \\
\hline Tensile Yield Strength ${ }^{*}(\mathrm{MPa})$ & $26-33$ & $21-28$ \\
\hline Tensile Ultimate Strength* (MPa) & $22-31$ & $39-48$ \\
\hline Tensile Ultimate Elongation* ${ }^{*} \%$ ) & $10-1200$ & $350-525$ \\
\hline Impact Strength, Izod* ( $\mathrm{J} / \mathrm{m}$ of notch; $3.175 \mathrm{~mm}$ thick specimen) & $21-214$ & $>1070$ (No Break) \\
\hline Degree of Crystallinity (\%) & $60-80$ & $39-75$ \\
\hline
\end{tabular}

*Testing Conducted at $23{ }^{\circ} \mathrm{C}$.

UHMWPE's microscopic structure is visualized using a transmission electron microscope (TEM) which reveals an interconnected network of crystalline lamellas embedded within amorphous regions (Figure 4). A schematic morphology of UHMWPE is also illustrated in Figure 5 showing that disordered regions may be connected with surrounding lamellae by tie-like molecules [43]. 


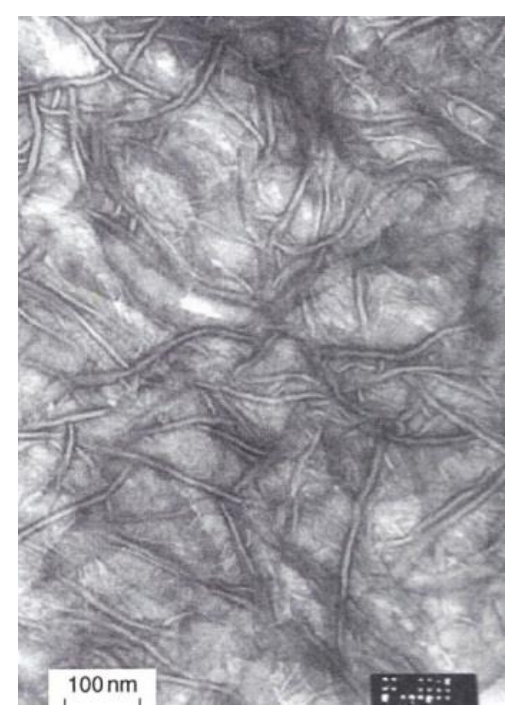

Figure 4. A TEM image of UHMWPE showing amorphous (gray color) and crystalline regions (white lines with a dark out-line) [43].

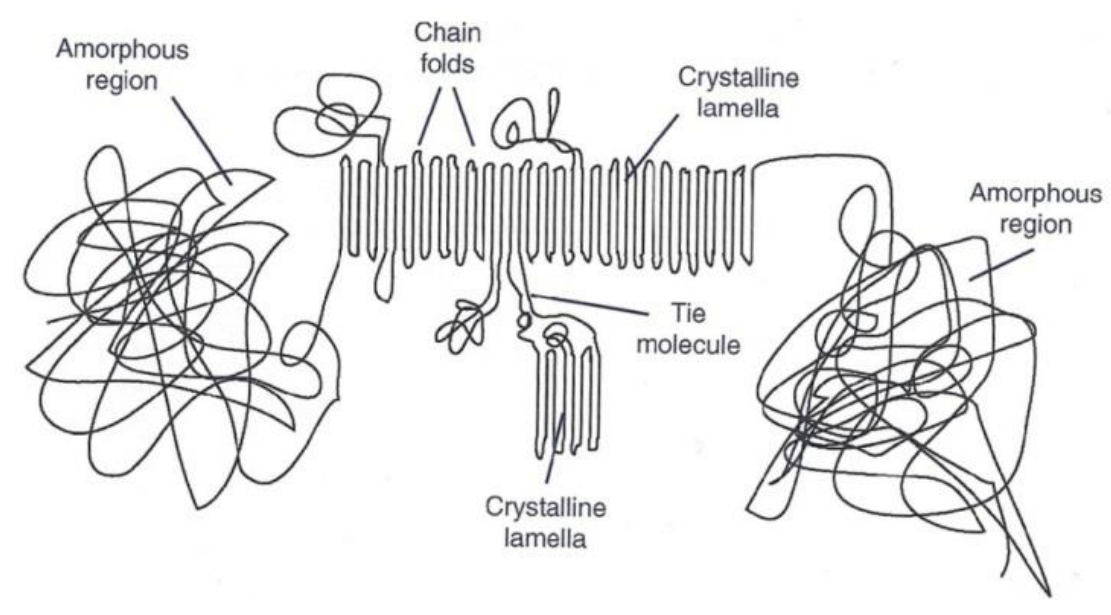

Figure 5. A schematic of the morphological features of UHMWPE [43].

Spectra ${ }^{\circledR}$ and Dyneema ${ }^{\circledR}$ are two commercially produced UHMWPE fibres on the market. The limitations of UHMWPE include its low operating temperature (less than $70{ }^{\circ} \mathrm{C}$ for shortexposure and $130{ }^{\circ} \mathrm{C}$ for long-exposure, Dyneema $\left.{ }^{\circledR}\right)$, creep susceptibility $(\sim 0.006-0.02 \%$ day at $30{ }^{\circ} \mathrm{C}$ and $300 \mathrm{MPa}$, Dyneema $\left.{ }^{\circledR}\right)$, and poor compressive strength ( $0.1 \mathrm{GPa}$, Dyneema $\left.{ }^{\circledR}\right)$ [40]. UHMWPE fibre $\left(\right.$ Spectra $^{\circledR}$ and Dyneema ${ }^{\circledR}$ ) is a good candidate to be used as a reinforcing material in composites as it has a low density $\left(0.97 \mathrm{~g} / \mathrm{cm}^{3}\right)$, high specific modulus $(\sim 70-120$ $\mathrm{GPa})$, and high strength $(\sim 2.3-3.5 \mathrm{GPa})$. On the other hand, the excellent chemical inertness 
against most chemical materials (e.g. organic acids, inorganic acids, bases and etc.), low surface energy $\left(\sim 27-31 \mathrm{~mJ} / \mathrm{m}^{2}\right)$, and smooth surface of UHMWPE fibres does not allow for a good adhesion to a matrix. This behaviour leads to a poor mechanical performance of such a composite. Currently, chemical etching and plasma treatment are the only methods to activate the surface of UHMWPE fibres [44-46].

\subsubsection{Aramid}

Aramid fibre was first developed commercially by DuPont during the 1970s under the trade names of Kevlar ${ }^{\circledR}$. Later, other commercial products of the aramid fibres became available in market under the trade names of Twaron ${ }^{\circledR}$ and Technora ${ }^{\circledR}[40]$.

$\operatorname{Kevlar}^{\circledR}$ is an organic material in the para-aramid class of aromatic polyamide (aramid) family. The primary chain of aramid is shown in Figure 6. Kevlar-29, Kevlar-49, Kevla-129, and KevlarKM2 are the latest Dupont products used in armor applications produced by modifying the primary chain of $\operatorname{Kevlar}^{\circledR}[14,40]$.

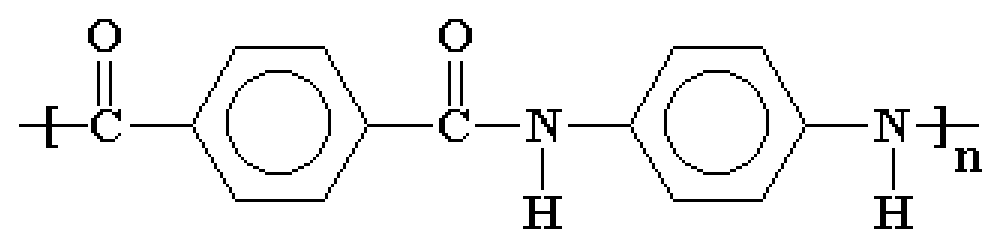

Figure 6. Aramid chemical structures [10].

$\operatorname{Kevlar}^{\circledR}$ has a unique combination of high strength, low weight, low electrical conductivity and low thermal shrinkage, high modulus, high toughness, high cut resistance, high thermal stability, and a good fatigue and creep resistance. Also, Kevlar ${ }^{\circledR}$ does not melt, soften, or flow and it is also flame resistant. In general, aramid fibres lose tensile strength by exposing to heat, moisture, or ultraviolet light, although, they have a good chemical stability [10, 38]. 


\subsection{Mechanisms of Impact Energy Dissipation in Multilayer Textiles}

There are various factors that may affect the performance of a multilayer textile target against different kinds of weapons: the areal density of fabric, the yarn linear density and fibre mechanical properties, the number filaments per yarn, the fabric weave architecture, the number of fabric plies; the boundary condition of the fabric and inter-connection conditions of plies; the geometry, type, mass, the speed of the weapon; and inter-yarn and fabric-projectile frictional characteristics within such a structure [29,30,47]. Considering a single yarn of para-aramid or UHMWPE under the strike of a projectile, two main modes of failure can be observed: (i) Transmitted transverse stress wave (TSW) failure mode at a low impact energy and (ii) Shear failure mode at a high impact energy [13]. Since energy waves can travel the farthest down the length a fibre with high elastic modulus, a large proportion of its volume can absorb the energy of a ballistic impact. Consequently, the impact energy can efficiently dissipate across a network of fibres [40]. For the same reason, a fibre with a high tensile strength and elongation to failure can absorb a considerable amount of energy via plastic deformation and stretching under an impact $[40,47]$.

\subsubsection{Effect of Frictional Force}

In general, a high strength and flexible multilayer textile target can provide considerable protection against various projectiles through a combination of different mechanisms: (i) Yarn pull-out from the fabric (i.e. yarn uncrimping, yarn translation, and lateral sliding) and (ii) Yarn failure (i.e. plastic deformation and fracture) $[13,33,48,49]$. The yarn pull-out effect accounts for the majority of impact energy absorption by frictional force (i.e. projectile-fabric and interplay friction), while the projectile can be defeated without fracturing yarns $[48,50]$. 
Under low impact velocity of a small and smooth projectile or in the case of loosely woven fabrics, the yarn pull-out mechanism (i.e. uncrimping, translation, rotation, and lateral sliding of fibres) plays a dominant role $[27,28]$. On the other hand, under the condition of either a blunt projectile with a high impact velocity or high constraint boundaries or a tightly woven fabric, plastic deformation and fibre breakage are the dominant mechanisms [33,48]. More specifically, yarn translation becomes more significant if only few fabric layers are present, while yarn uncrimping is the most prevalent in a target with a high number of fabric layers [48]. When the pull-out effect is not considerable, the role of mechanical properties of the fibre becomes more significant [51]. In other words, unlike yarn sliding and windowing effects, the extent of compression, shear, and transverse failure are more directly related to the mechanical properties of fibres [34].

Instead of pushing the fibres aside, a projectile can break more of the fibres if the inter-yarn and fabric-projectile frictional force is high enough, which results in increased energy absorption of the fabric [11,34]. Consequently, any treatment that may increase inter-yarn friction can enhance the ballistic performance of the fabric [11]. The frictional characteristics of a multilayer fabric can be modified through frictional treatment, lubrication, inter-yarn bonding, or coating [51]. Under certain conditions, it was suggested that yarn's mobility should be tuned to achieve the highest penetration resistance. The highest penetration resistance may be possible by suppressing the windowing effect and having enough fibre slippage. This slippage reduces an instantaneous stress concentration on yarns and filaments in the vicinity of the impact, preventing a premature yarn failure [34].

Three distinct modes of failure can be identified from a quasi-static penetration test on fabrics: (i) Local yarn failure, (ii) Remote yarn failure, and (iii) Yarn pull-out [12]. This slow penetration 
test helps observe the failure evolution of fabric and quantitatively measures load and deflection [25]. The yarn pull-out test is a legitimate analysis of inter-yarn and yarn-impactor frictional properties. In this test, the fabric target is pre-tensioned in a transverse direction to that of the yarn being pulled. The role of frictional force is then estimated from force-displacement curves measured in this yarn pull-out test event $[8,12,34,48,49]$, and simulates yarn slippage [52]. The yarn pull-out mechanism represents a frictional force between intersecting perpendicular yarns and does not involve fibre breaking [12]. Although, the pull-out test is conducted at a low velocity, assuming that the yarn pull-out is the dominant energy absorption mechanism, its results can be quantitatively correlated with the actual yarn pull-out during a ballistic impact [48].

The area under a yarn pull-out force-displacement curve represents the total energy required during the pull-out test. The higher this energy, the larger the penetration resistance and energy consumption during a frictional action among yarns [12,52,53]. The total transferred energy to a fabric layer under an impact can also be estimated from the pull-out test which is directly related to the energy of both transverse and longitudinal generated waves per unit length of the yarn, the number of yarns pulled-out, and the pull-out length [50]. Also, this transverse wave propagation during a ballistic impact is the reason for the formation of a cone on the back face of the target [54].

\subsubsection{Effect of Various Coating Materials}

Any type of coating on a fabric can alter its yarn-yarn and ply-ply frictional force. As a result, the energy absorption of a multilayer structure of coated fabric is not the same as the uncoated one [55]. A thermal-sprayed hard ceramic and cermet coating on high strength Twaron $^{\circledR}$ fabric can increase its original ballistic resistance, although the weight increase of the coating is 
significant. It was interpreted that such a ceramic coating increases fibre-fibre frictional force and prevents wave distortion and delamination. In results, such a coated fabric help dissipates and damp the shock wave velocity of a penetrator [7].

A multilayer stack of Twaron ${ }^{\circledR}$ fabric soaked in a colloidal silica dispersion shows a higher ballistic resistance, upon removal of the water medium, than a multilayer stack of neat Twaron ${ }^{\circledR}$ fabric. The reason of this improvement is the presence of silica particles and the formation of silica clusters which increases projectile-fabric and inter-yarn frictional force. On the other hand, the specific ballistic energy of the system was reduced by this treatment due to the considerable weight gain [11]. Similarly, treating $\operatorname{Kevlar}^{\circledR}$ fabric with dry particles of polymethyl methacrylate (PMMA) or silica can improve its puncture/ballistic resistance [34].

A multilayer stack of $\operatorname{Kevlar}^{\circledR}$ fabric which was laminated and infused with a thermoplastic film (e.g. Surlyn ${ }^{\circledR}$ or polyethylene) can provide a higher cut/puncture resistance than a multilayer stack of neat $\operatorname{Kevlar}^{\circledR}$ fabric. This improvement was achieved due to the increased restriction in fibre motions, i.e. increasing the windowing resistance by enhancing surface tension and frictional force between fibres. Hence, a higher energy was required to cut and fracture this thermoplastic coated system, although the flexibility would be compromised after this treatment $[5,8]$.

A $\operatorname{Kevlar}^{\circledR}$ fabric coated with natural rubber latex (NRL) and carbon nanotubes (CNT) can provide a higher puncture resistance than a neat $\operatorname{Kevlar}^{\circledR}$ fabric. Similar enhancement was also reported for an NRL coated nylon or Twaron ${ }^{\circledR}$ fabric [56]. A UHMWPE fibre unidirectional (UD) non-woven fabric coated with NRL can also provide a higher puncture resistance compared to the uncoated UHMWPE UD fabric, regardless of the huge increase in areal density after coating. This puncture resistance enhancement was also attributed to the higher yarn-yarn 
and yarn-probe frictional force [57]. Similarly, the ballistic performance of an NRL coated Twaron $^{\circledR}$ fabric is superior to the uncoated one, regardless of considerable increase in thickness and areal density of the fabric after coating [58,59].

Nylon coating is another technique to increase the penetration resistance and mechanical properties of inherently inert UHMWPE fibre/fabric through an interlocking mechanism. The improved puncture/stab resistance of such a nylon coated UHMWPE fabric was interpreted to the restrained fibre motion and limited fibre pull-out [60-62]. The mechanical properties (e.g. tensile strength and stiffness) of polyurethane coated nylon fabric were also measured and analyzed under different conditions [63].

\subsubsection{Effect of Boundary Condition and Stitching}

The effects of test conditions, such as the effect of boundary condition and gripping/clamping type of the fabric, on the penetration performance of textile-only and textile-STF structures have been investigated in several studies. It was reported that the effect of test conditions is more influential when the impact velocity of the projectile is relatively low or the size of fabric target is small $[32,42,53,64]$.

From the ballistic tests and computational models, it was reported that a stack of multilayerTwaron ${ }^{\circledR}$ fabric showed higher impact energy absorption if the sample target was clamped at two of its four edges compared to when clamped at all four edges. The lower energy absorption of the target with four edges of clamped boundary constraints was attributed to the premature failure of the primary yarns at the impact area due to the reflection of strain waves when they reached a fixed edge. On the contrary, if the ends of yarns were free, the yarns could pull inward easily in response to the impact [64]. The yarn orientation with respect to the 
direction of the clamped boundaries is another influencing factor in the ballistic performance of the fabric target [64].

In general, certain types of stitching could improve the ballistic resistance of a stack of multilayer fabric to some extent $[55,65,66]$. This was interpreted to better inter-ply interaction of the stitched fabric which could help trap the projectile easier [55], however, this improvement was achieved with the expense of an increase in areal density [66] and flexibility [55] of the system. For a textile composite system, such as vinyl ester resin matrix reinforced with E-glass fibres, it was reported that through-the-thickness stitching with the Kevlar ${ }^{\circledR}$ yarn help enhance the resistance against explosive blast. It was interpreted that such a Kevlar ${ }^{\circledR}$ stitching could improve Mode I interlaminar fracture toughness [65].

\subsection{Textile/resin Composites for Advanced Protection}

The polymer matrix composite (PMC) reinforced with woven or non-woven high strength fibres have been used for lightweight vehicle armor and protective helmet applications. The ballistic performance of such a composite depends on various factors: the properties of fibre and resin, the fibre structure, the fibre volume, and the compatibility of fibre with resins and additives [40]. In general, the ballistic performance of a textile-only structure is superior to the textile-resin composite structure; even though the textile-only system is significantly lighter than the latter one with equivalent number of fabric layers [13]. The low ballistic performance of a textile-resin structure was interpreted to the restriction of the deformation ability which limits its ballistic resistance [40].

A laminar composite of $\operatorname{Kevlar}^{\circledR} /$ Polyvinyl Butyral (PVB) can provide a considerable ballistic resistance when moulded at a high temperature and pressure. The high energy absorption of such a composite system is due to the combination of a strong aramid fabric and a ductile 
thermoplastic matrix [67]. In the past, UHMWPE fibre reinforced UD plate has been widely used in protective applications. The ballistic performance of this composite could be enhanced significantly by adding conch shell particles in the resin matrix. Hence, more energy could be absorbed through a delamination (interface debonding) mechanism and the initiation of microcracks within the resin in the event of a ballistic impact [68].

\subsection{Shear Thickening Fluids (STFs)}

\subsubsection{Shear Thickening Phenomenon}

The micromechanics of shear thickening phenomenon was first explored over four decades ago [69-72]. The shear thickening behaviour of a variety of different colloidal dispersion has previously been investigated with regard to the effect of particle concentration, particle size distribution, particle-particle interactions, and viscosity of the dispersing medium. For instance, when the concentration of hard particles in a colloidal dispersion is increased or size distribution of the particles is reduced, the critical shear rate $\left(\dot{\gamma}_{c}\right)$ shifts to a lower value and the maximum shear thickened viscosity $\left(\eta_{\max }\right)$ increases [73-77]. As another example, all types of surfactants can alter the rheological properties of STF materials by changing either of surface charges or interparticle interaction [78].

The shear thickening behaviour of a concentrated near-hard sphere colloidal dispersion is characterized by two widely accepted mechanisms: (i) Hydrodynamic forces cause the formation of transient jamming shear-induced hydroclusters that bond together and increase the viscosity of the dispersion abruptly (hydroclustering) [72-75,79-82] and (ii) In-layer order of particles transforms into a disorder state, and viscosity increases due to an increase of hydrodynamic attractive forces on the particles (order-disorder transition, ODT) [77,83]. Under some certain 
conditions, both events may occur at the same time, which means that the formation of hydroclusters facilitates the disruption of an ordered-layer structure $[73,84]$.

The schematic of the change in the microstructure of a colloidal dispersion is shown in Figure 7, showing a transition from shear thinning shear thickening. At the equilibrium, there is a random collision between the particles while they can resist naturally with the flow. The presence of an in-layer order state of the particles can explain the shear thinning phenomenon as the particles became more organized and permitting the flow with fewer interparticle encounters. At higher shear rates, transient hydroclusters are formed as the hydrodynamic interactions between particles became dominant, which results in an abrupt increase of the viscosity (shear thickening) $[85,86]$.

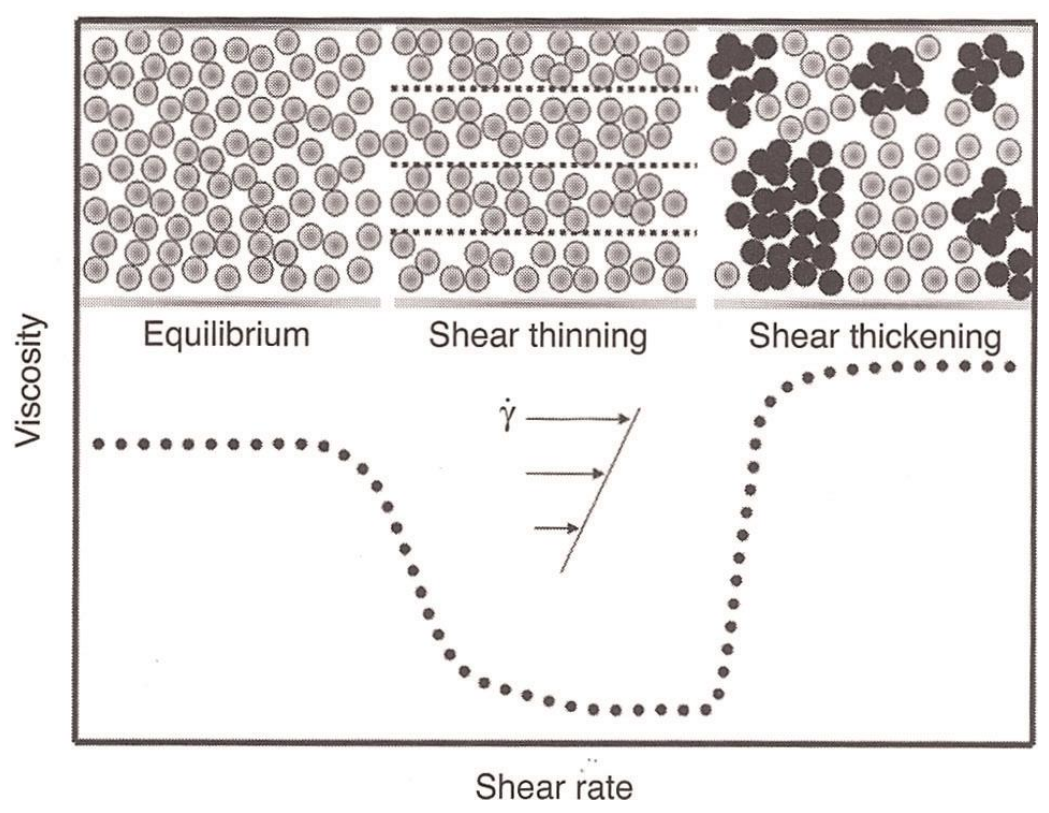

Figure 7. A schematic of the change in the microstructure of a colloidal dispersion with a shear rate(the dark particles represent hydroclusters) [85].

\subsubsection{Applications for STF Materials}

The possible applications for shear thickening fluid (STF) materials can be categorized into three distinct classes: (i) Liquid body armor and protective shields, (ii) Devices with adaptive stiffness 
and damping, and (iii) Smart structure [15]. For an example, it is suggested that STF-fabric composite materials can potentially be used in spacecraft to provide a protective shield against hypervelocity particles [87]. The potential protection against hypervelocity impact threats was experimentally and computationally examined $[88,89]$.

The STF materials can change their properties with changing loading conditions, providing a self-limiting maximum rate of flow mechanism and a unique combination of increased energy dissipation and increased elastic modulus. As a result, STF materials are favorably used for speed control devices, clutches, as a damping mechanical element, and shock-absorbent [86,90]. In this regard, a mathematical model was developed to address the working mechanism of STF materials in an STF-based prototype of a speed-activated smart damper device [91]. Also, the potential application of STF materials in a sandwich composite structure which exposed to dynamic flexural deformations was studied to modify its vibration and damping response [92]. The potential use of STF materials in body limb movement limiter devices is also patented to restrain the movement of a person's arm, shoulder, lower leg, and knee [93]. The main application of STF materials, i.e. for liquid body armor, will be explained in Section 2.4.3.

\subsubsection{Textile-STF with Advanced Protective Performance}

Liquid body armor is a novel composite technology composed of a non-Newtonian STF material impregnated within a multilayer stack of high performance fabric. The incorporation of STF materials onto fabrics to enhance their ballistic resistance has attracted much interest in the past decade. The key advantage of this novel armor is that a highly viscous STF material immediately transits into a solid-like state under an impact force making the permeated fabric stronger, and reverts to its viscous gel-like state again shortly afterward remaining flexible during normal 
wear. The application of STF materials to enhance the penetration resistance of Kevlar ${ }^{\circledR}$ fabrics was studied in several research studies [1,2,9,10,16-34,37,38,52,94].

The use of STF materials with high performance fabric for armor application is not limited to Kevlar $^{\circledR}$. Several researchers investigated the incorporation of STF materials onto various fabrics, such as nylon [1,3,24,27,38], UHMWPE [39], and glass [36]. Providing a similar range of penetration resistance, an STF-impregnated target is even thinner and more flexible than the textile-only system of the same areal density. Therefore, the number of fabric layers in a body armor is reduced [1-3,38,39]. Moreover, liquid body armor could potentially provide resistance against both ballistic and stab treats (i.e. a multi-threat protection) [2]. Additionally, an STFKevlar or nylon-STF system can provide enhanced resistance against a conventional threat of syringe needles, and can be used for medical protective clothing [27].

\subsubsection{Mechanism of Enhanced Penetration Resistance of Liquid Body Armor}

The high level of penetration resistance in liquid body armor is interpreted to the reduction of its yarn mobility within fabric, relative to the impactor and other yarns, and higher yarn-yarn frictional force which increases yarn pull-out force from the fabric [1,2,21,33,34,52,53]. Because of increased inter-yarn friction, the failure mechanism of STF-treated fabric is more related to the breakage of fibres and yarn rather than fibre slipping or windowing effect, opposite to the dominant mechanism of textile-only structures [17,34].

In textile-only structures, fibres could be spread-apart and are easily pushed away to create an open area for a penetrator to go through (i.e. the windowing effect) [34], although, STF materials can bond yarn together and suppress their motion, sliding, and separation. Therefore, yarn can engage more effectively with an impactor [1-3,24,25,27,30,33]. 
Because of enhanced inter-yarn friction, more of the impact load can be spread to the adjacent yarn of the fabric. Hence, such a fabric is not directly impacted by the projectile [34]. Moreover, the observation of an extensive fibre breakage with minimized fibre pull-out near the impact point of a textile-STF target could be indicative of enhanced penetration of such a system. This means that the fibres were loaded effectively to an ultimate failure strength with minimized yarn motion in the event of impact prior to the significant yarn pull-out [33]. STF materials can also provide a better coupling and a load transferring mechanism between yarn or fabric plies, which allows the whole fabric structure to be loaded more efficiently [21].

In general, textile-STF structures can provide a better puncture resistance than cut resistance as the decreasing yarn mobility can hardly influence the global cut resistance. Consequently, a knife can cut filaments and penetrates through them without pushing fibres aside [1-3]. In other words, cutting damage is highly localized $[52,94]$. Therefore, a failure with blades can be correlated to the strength of yarns rather than inter-yarn friction [52].

Moreover, the effect of fabric architecture, i.e. yarn denier and yarn count, on the cut resistance of textile-STF composites is negligible [3]. In general, a high Denier fabric, such as "DuPont Correctional Kevlar ${ }^{\mathbb{B} ”}$, can provide a higher cut resistant than the same fabric with lighter Denier yarns, due to the prevention of an early failure [3,51]. Also, the puncture resistance of fabric increases by increasing its yarn-count because as it provides more restraint on the mobility of individual yarns; which is also analogous to the effect of increasing STF add-on in a textile-STF structure $[2,3,27]$.

There are two interpretations that can relate the increase of yarn pull-out resistance to the increase of energy dissipation in textile-STF structures: (i) The less total fibre pull-out is required to absorb impact energy because more force is required to pull-out each individual fibre and (ii) 
Adsorption of additional energy through fibre deformation and fracture because of the increased fibre pull-out resistance [10].

From fibre pull-out testing it was observed that the force increased with the increase of STF add-on over individual filaments. More STF add-on increases inter-yarn frictional force and improves the impact energy absorption of the STF-textile by suppressing windowing and fibre pull-out effects $[9,10,16]$. Since fibre pull-out force can be increased by increasing the speed of pulling the fibres out of fabric in a textile-STF structures, shear thickening phenomenon is considered accountable for such an enhancement. On the contrary, fibre pull-out force is nearly independent of the test speed in a neat fabric sample [26,52]. The significant effect of inter-yarn and fabric-projectile frictional force have been studied in several STF-related studies $[2,9,10,16,26,29,30,33,34,52,53]$.

Another plausible explanation for the advanced performance of liquid body armor is that an STF material can absorb the impact energy by itself due to the viscous dissipation of this nonNewtonian fluid [21]. The penetration resistance of a textile-STF target can be improved by optimizing the impregnation process of STF materials. This means that a more uniform, deeper, and denser compact distribution of an STF material should be provided onto individual filaments with even a lower STF add-on $[9,16]$.

Since the inter-yarn frictional force of textiles increases with STF treatment, the apparent modulus of each individual yarn is consequently increased. Hence, the stacks of hybrid layers of STF-impregnated Kevlar ${ }^{\circledR}$ fabric with neat $\operatorname{Kevlar}^{\circledR}$ could show enhanced ballistic resistance if the STF-impregnated fabrics were stacked behind neat ones. Upon an impact, this sequential lamination causes the synchronized elongation of facing yarns in the frontal fabric layers and restricted elongation of highly-tensioned STF-impregnated fabric layers on the rear side [30]. 
The penetration resistance of a textile target can be degraded if it is impregnated with water or a Newtonian fluid, such as polyethylene glycol (PEG) or ethylene glycol (EG). The reason for such degradation is that the inter-yarn frictional force becomes lower and intensive yarn pull-out and windowing effects are detected [21,33,34,39,52].

\section{Water Absorption; A Drawback of Using STF Materials}

STF materials are prone to water absorption and evaporation which impacts their properties $[15,25]$. These materials can be easily washed away with direct exposure to water or solvents [24]. Several methods have been suggested to overcome this drawback in textile-STF composites. For instance, the stacks of hybrid layers of STF-impregnated Kevlar ${ }^{\circledR}$ fabric with neat Kevlar $^{\circledR}$ plies infused with a thermoplastic film can provide high puncture and stab resistance. In addition, the stacking of fabric layers impregnated with Poly(ethylene-comethacrylic acid), also known as Surlyn ${ }^{\circledR}$, on top and bottom layer of a fabric layer impregnated with STF material can provide a water and moisture resistant shield to the STF [24]. Another proposed method is to construct a multi-component structure consisting of a hollow fibre filled with an STF material [95]. Practically, this patented technique could prove difficult to pursue because of difficulties in pumping STF materials into the hollow space of fibres [15].

On the contrary, without using an STF material, the effect of water absorption on the ballistic performance of textile-only target is not significant [66]. For an example, it is reported that the effect of water absorption on the puncture/stab resistance of a nylon coated UHMWPE fabric is negligible [62]. In general, the ballistic resistance of a wet multilayer aramid fabric is lower than the dry one, which is likely because of the lower inter-yarn and ply-to-ply frictional force in wet samples [50]. 


\section{Influence of STF Characteristics in Liquid Body Armor}

The hardness of the submicron size particles used to fabricate STF materials plays an important role in the penetration resistance of the liquid body armor. The impregnated Kevlar ${ }^{\circledR}$ fabric with an STF material made of soft polymethyl methacrylate (PMMA) particles showed lower stab/ballistic resistance compared to an impregnated Kevlar $^{\circledR}$ fabric with much harder silica particles. It was interpreted that hard particles can significantly abrade the fabric in the vicinity of the impact which provides a better STF-fibre and fibre-fibre interlocking mechanism and limits the yarn mobility. Another plausible explanation is that the hard particles could embed more effectively into the soft polymer filament and increase the direct mechanical coupling and load transferring between fibres and particles. In addition, more fibre breakage and fibre fibrillation were detected in the case of silica-STF impregnated Kevlar $^{\circledR}$ fabric and little windowing effect or fibre pull-out. By contrast, the windowing effect and fibre pull-out remain the primary failure modes in neat $\operatorname{Kevlar}^{\circledR}$ fabric or PMMA-STF impregnated $\mathrm{Kevlar}^{\circledR}$ fabric. There is also some evidence to suggest that the soft PMMA particles can accommodate stress via particle deformation $[3,22,23,34,52]$.

The use of platelet-like hard particles (e.g. Kaolin) of the same average size and volume fraction of particles is preferable than spherical shaped silica particles to make an STF-textile composite resistant to small size threats like needles. An STF material comprised of oblate Kaolin ellipsoids could provide a more efficient geometry to laterally distribute the stress from a force point

source. On the other hand, the kaolin-based STF material impregnated with Kevlar ${ }^{\circledR}$ fabric showed a continuous shear thickening behaviour which resulted in poor stab/ballistic protection [37]. 
The size of the particles used to make STF materials plays a minor role in the performance of STF-textile composite, as long as the particles are small enough to be inserted between the filaments, which means that the relative size of particles to the filament diameter and interstitial volumes is small [34].

On the other hand, it was reported that Using a smaller size of hard-spherical silica particles (average particle size of $100 \mathrm{~nm}$ versus average particle size of $500 \mathrm{~nm}$ ) to fabricate STF materials can slightly enhance the ballistic performance of the STF impregnated Kevlar ${ }^{\circledR}$ fabric due to the increase of the inter-yarn and yarn pull-out frictional force at the onset of shear thickening resulting, which results in the greater energy dissipation of the impact [53]. In general, the critical shear stress and, hence, the critical shear rate at the onset of shear thickening behaviour of a hard spherical particle dispersion scales inversely with particle size cubed [96]. It was reported that the shear thickening strength of a final STF product upon removal of alcohol from a silica/PEG/alcohol mixture could be improved by a sonochemical mixing technique, therefore, an extra puncture/stab resistance was added to the STF-textile system [38,76]. Use of a silane coupling agent to functionalize the silica nanoparticles used in the fabrication of STF material can provide a considerable favorable impact on the puncture resistance of STF-textile composites. It was reported that the puncture resistance of such a composite was increased by using a coupling agent due to the formation of additional siloxane bonds between silica particles and a PEG dispersing medium. Also, the adhesion of the STF material to individual filaments could be improved by such a treatment on silica particles which provides a superior coating coverage of nanoparticles on the surrounding yarns and filaments [87].

Various short, discontinuous high strength fibres (e.g. carbon, glass, and HDPE fibre) can be blended with an STF material to enhance the ballistic performance of STF-textile composites. It 
was shown that the addition of these short fibres increased the squeeze flow resistance of the STF material. This flow resistance was measured by squeezing the sample in a parallel plate geometry of rheometer and recording the change of normal force as a function of time. It was also mentioned that such a rheological technique could be indicative of some of the flow dynamics which happens during a realistic ballistic impact [97].

The addition of such a short fibres can also provide enhanced tensile strength to the STF material and promotes load transferring through the STF-fabric composite in the event of impact. It could be anticipated that such a composite is capable of providing enhanced ballistic, stab, and shock resistance [98]. Moreover, the energy absorption of the STF-textile composite can be increased by increasing the particle loading which improves the consequent shear thickening response. This behaviour emphasizes the importance of shear thickening response in the performance of liquid body armor [21]. Also, a more highly concentrated STF can provide better yarn bridging

with a coherent structure at the time of impact [17]. It was shown that the most effective penetration can be achieved with the full discontinuous shear thickening property of the STF [33].

\subsubsection{Influence of Ultrasonication on the Viscoelastic Characteristics of STF}

As a general requirement, the volume fraction of solid particles in a non-flocculated suspension must be high enough to exhibit shear thickening behaviour. Shear thickening flow behaviour is where the viscosity of a fluid increases abruptly with increasing shear stress or shear rate. Shear thickening is generally observed in a wide variety of concentrated colloidal dispersion of hard particles [72-75,84,99].

The incorporation of non-Newtonian shear thickening fluid (STF) materials onto fabrics to enhance their ballistic resistance has attracted much interest in the past decade (see Section 
2.4.3). The viscoelastic characteristics and gel-stability of various fumed silica dispersions have been extensively studied $[26,73,84,100-104]$. In general, there are many widespread applications that use fumed silica particles in different dispersing liquids because of its unique ability to form a three-dimensional particle network [84,105-108].

The extreme hydrophilic property of fumed silica is rendered by the presence of hydroxyl groups on the surface of the particle. The silanol $(\mathrm{Si}-\mathrm{OH})$ groups are generated during the preparation of fumed silica particles by hydrolysis of silicon tetrachloride $\left(\mathrm{SiCl}_{4}\right)$ in a flame of $\mathrm{H}_{2}$ and $\mathrm{O}_{2}$ $[73,84,105,106,109]$. Silanol groups may present on the surface or throughout the silica structures. The density of silanol groups is not dependent on the specific area of the particles, although, the distribution of silanol groups varies with the particle surface area [109].

The rheological properties of fumed silica dispersion depend on three distinct factors: (i) Concentration, (ii) Degree of hydrophilicity or hydrophobicity of the particles and the physiochemical nature of the particle surface, and (iii) Polarity of the dispersing liquid medium. The fumed silica dispersion can turn into a gel state if the interparticle linkages extend across its volume. A three-dimensional network of particles is eventually created which compromises the stability state of the dispersion $[105,106]$. In general, high intensity ultrasonic baths are used to remove air bubbles from a highly viscous dispersion and to improve its homogeneity just before conducting rheological tests $[100,102,103,107,108]$.

Moreover, it was suggested that a few minutes of ultrasonication is an effective treatment to rejuvenate a weak gel state by disrupting its structure, although, no further detail was provided [103]. In general, this technique was used to enhance a sonochemical process (e.g. polymerization) or to accelerate a heat or mass transfer process (e.g. dispersion). The 
sonochemical process can be explained by the creation of numerous cavities in a liquid medium during a chain reaction of sonic shock waves [38,76,110-113].

\subsection{General Discussion}

A general overview of the development of only-textile body armor and liquid body armor was provided in the preceding sections. This chapter started with a critical review about the development of synthetic high strength fibres for the manufacture of protective clothing. In Table 2, various mechanical properties of glass, aramid, UHMWPE, and PBO fibres were listed and compared. In Section 2.2.1, a review of the mechanical and physical properties of UHMWPE polymer was provided. In Figure 4, a TEM image of UHMWPE polymer was provided and a schematic of the structure of this polymer is shown in Figure 5.

The chemical structure of aramid fibres was shown in Figure 6, with a brief review of the mechanical and physical properties of this polymer (Section 2.1.2). A critical review of the failure mechanisms of textile body armor was provided in Section 2.2. From the literature surveys provided in Section 2.2.2, it was concluded that coating is an applicable treatment to improve the penetration resistance of textile body armor against various types of threats.

Next, liquid body armor was introduced as a novel composite technology, composed of a nonNewtonian STF material impregnated within a multilayer stack of high performance fabric (Section 2.4). More specifically, the shear thickening phenomenon was explained in Section 2.4.1, and a review of the available and potential applications of STF materials was provided in Section 2.4.2.

Finally, a literature review of the mechanisms of enhanced penetration resistance of liquid body armor and the influence of STF characteristics was presented in Section 2.4.4, followed by a review of the viscoelastic properties of fumed silica dispersions (Section 2.4.5). It could be 
gleaned from this literature review that the successful use of inherently inert UHMWPE polymer for liquid body armor application has not yet been introduced.

From the comprehensive literature review in this chapter, it was revealed that only a few studies have been conducted to evaluate the stability of STF materials for high impact applications, and even fewer studies have been conducted to examine the viscoelastic characteristics of fumed silica colloidal dispersions. The main objective of this research, therefore, is to develop a new composite material, based on UHMWPE, to overcome the limitations of current security applications including failure to stop punctures and stab threats, high cost, and bulky weight. 


\section{CHAPTER 3 MATERIALS AND EXPERIMENTAL DETAILS}

This chapter presents the details of the experimental work performed in this study. In the first section, the preparation of nylon solution and the description of nylon coating technique onto fibres and fabrics will be provided. This nylon coating was applied onto inert UHMWPE fibre/fabric sample in order to improve its mechanical and physical properties. A description of different tests and measurements on the nylon coating under investigation will be provided, which includes: (i) Dynamic impact penetration test on fabric samples, (2) Quasi-static penetration test on fabric samples, (iii) Tensile and creep tests on fibre samples, (iv) Flexibility tests on fabric samples, and (v) SEM analysis. In the subsequent section, the details of the fabrication method of fumed silica dispersions will be discussed. This STF material is synthesized to integrate with a multilayer stack of nylon coated UHMWPE fabric sample in order to improve the penetration resistance of the textile structure against stab and puncture threats. A description of the sequential ultrasound technique will be provided to fabricate stable STF materials with controlled shear thickening property. Finally, the description of different tests performed on the synthesized STF materials under investigation will be provided which includes:

(i) A complete set of rheological studies (rotational and oscillation), (ii) Zeta potential measurement, and (iii) SEM analysis.

\subsection{Nylon Coating: A Novel Solution for Protective Clothing}

\subsubsection{Material Details}

Nylon 6,6 $\left(\mathrm{C}_{12} \mathrm{H}_{26} \mathrm{~N}_{2} \mathrm{O}_{2}, \rho=1.14 \mathrm{~g} / \mathrm{mL}\right)$ and $6,12\left(\mathrm{C}_{18} \mathrm{H}_{34} \mathrm{O}_{2} \mathrm{~N}_{2}, \rho=1.13 \mathrm{~g} / \mathrm{mL}\right)$ pellets were purchased from Sigma-Aldrich to coat UHMWPE fibre/fabric. Anhydrous methyl (MeOH), ethyl $(\mathrm{EtOH})$ alcohol, and ASC grade anhydrous calcium chloride $\left(\mathrm{CaCl}_{2}\right)$ were purchased from VWR International to dissolve nylon pellets. UHMWPE plain woven $21 \times 21$ ypi (yarns per inch) 
fabric with an areal density of $231 \mathrm{~g} / \mathrm{m}^{2}$ was supplied by Barrday Inc, Canada. A 1200 Denier UHMWPE fibre, consisting of 360 filaments, each with the diameter of $20 \mu \mathrm{m}$, was supplied by Montaki Canada Inc.

\subsubsection{Sample Preparation; Nylon Coated Fibre/Fabric}

\section{Nylon}

Synthetic polyamides, first invented in 1930s, are more commonly known as nylon. In general, nylon polymers possess high strength, high toughness, high elasticity, high luster, high abrasion resistance, high dye-ability, good shape-holding characteristics, and high chemical inertness, which make them suitable for many applications. There are several types of synthetic nylon polymer, such as $6 ; 4,6 ; 6,6 ; 6,10 ; 6,12$ and 12,12 [114].

Nylon 6,6, which was also used in this experiment, is the most widely utilized polyamides in market. There are two reactive monomers needed to synthesize Nylon 6,6 (Figure 8): (i) Hexamethylenediamine (HMD) and (ii) Adipic acid. The carbonyl oxygen on adipic acid and the amide hydrogen on HMD can hydrogen bond together and link to neighboring Nylon 6,6 chains (Figure 9) [115].

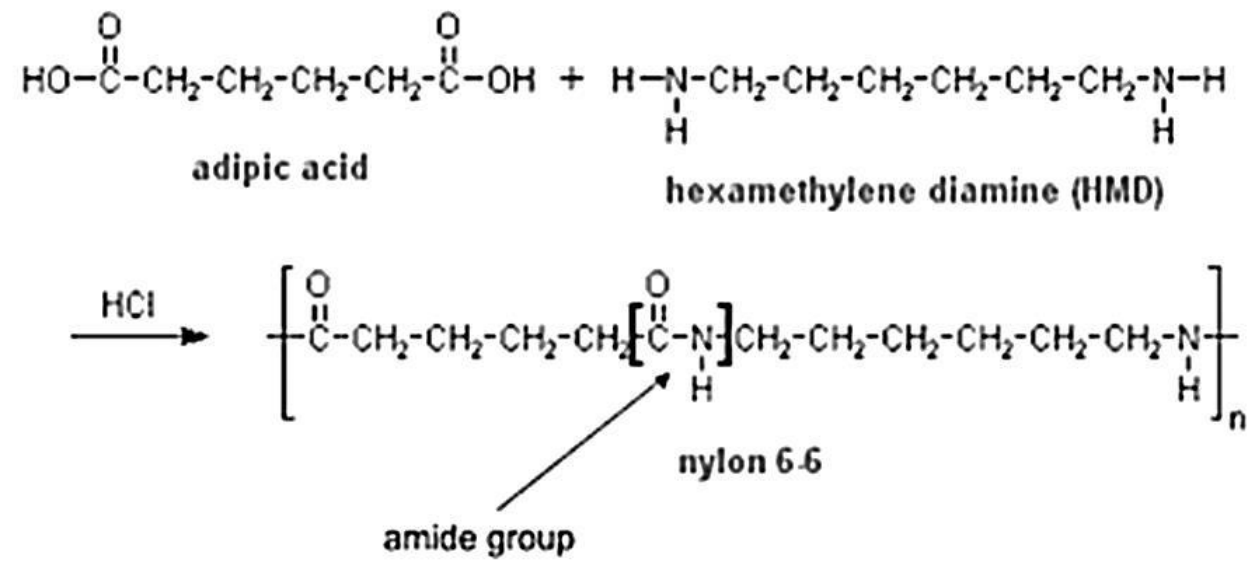

Figure 8. Chemical reaction to synthesize Nylon 6,6, Adopted from [115]. 


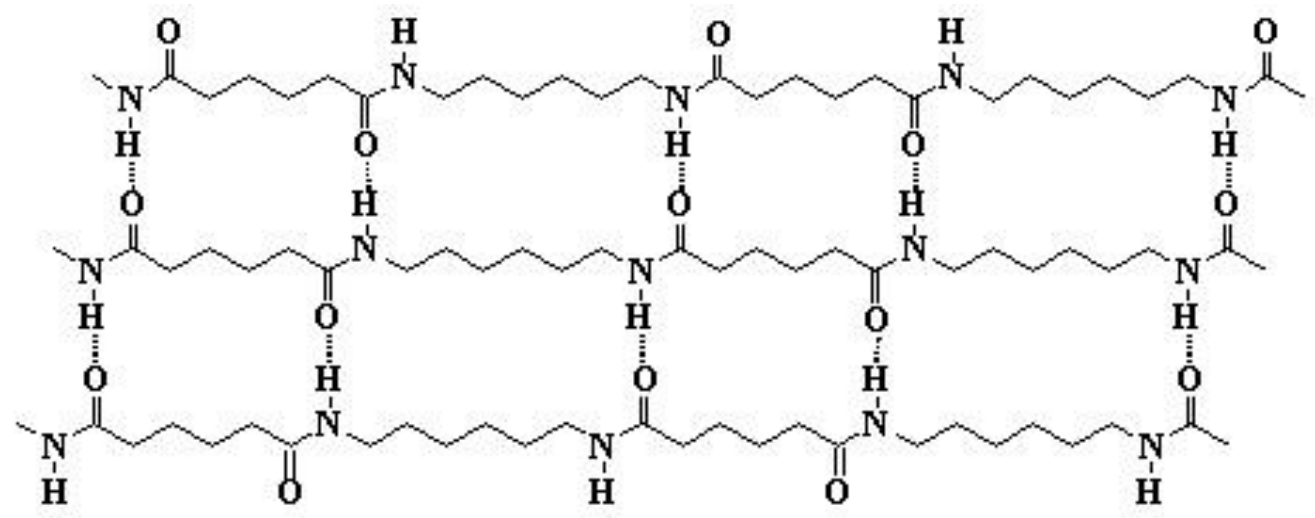

Figure 9. Structure of Nylon 6,6, Adopted from [114].

Nylon 6,12 is the other type of nylon which was used in this research. In general, Nylon 6,6 and Nylon 6,12 have a similar chemical composition, differing in only the length of the alkyl spacer chain structure of the acid component (6 carbons versus 12 , respectively) of the polyamide.

\section{Nylon Solution}

Nylon 6,6 and Nylon 6,12 pellets were dissolved in anhydrous $\mathrm{MeOH}$ and EtOH (respectively) in the presence of anhydrous $\mathrm{CaCl}_{2}$. The ratio of nylon to $\mathrm{CaCl}_{2}$ to alcohol in the solution was most preferably 1:5:21 (by weight) by exploring different weight fractions of the constituents. The solution was heated to the refluxing temperature of the alcohol for several hours in order to completely dissolve nylon pellets. It was observed that a lower concentration of $\mathrm{CaCl}_{2}$ in the solution resulted in an incomplete dissolution of nylon pellets. Another problem with an insufficient percentage of $\mathrm{CaCl}_{2}$ was that such a nylon solution did not remain soluble when cooled down to the room temperature and had to be reheated.

\section{Nylon Coating on Fibre/Fabric}

The UHMWPE fabric was cut to the desired size and then submerged in the prepared nylon solution for $30 \mathrm{sec}$. A manual rubber coated nip roller was used to squeeze out excess nylon solution from the fabric and ensure a reasonably uniform coating. The coated fabric sample was 
hung for several hours and then rinsed in distilled water for 5 min to remove residual $\mathrm{CaCl}_{2}$. The wet fabric sample was then hung overnight in a fume hood to dry completely prior testing. To coat the UHMWPE fibre, a spooled fibre sample was placed in line on a prototype automatic coating machine especially made for this research. The fibre was drawn using a DC motor operating at 60 RPM with a traveling speed of $0.1 \mathrm{~m} / \mathrm{s}$ for approximately $6 \mathrm{sec}$ through a holding tank $(65 \mathrm{~cm} \times 5 \mathrm{~cm} \times 2 \mathrm{~cm})$ containing the nylon solution. The wet coated fibre was then directed into two cone shaped dies in succession with $1 \mathrm{~mm}$ and $0.7 \mathrm{~mm}$ hole diameters, respectively, to remove excess material and create a uniform coating. The coated fibre was then drawn upward and downward several times to give enough time to dry the fibre with the help of multiple installed fans. The fibre was then drawn to a distilled, deionized water tank to remove $\mathrm{CaCl}_{2}$ from the nylon coating. Eventually, the coated fibre was spooled onto a second (and final) spool.

\subsubsection{Preliminary Evaluation of Nylon Coating}

\section{Flexibility Test and Thickness Measurement}

A flexibility test and a thickness measurement were performed to examine the impact of nylon coating to the UHMWPE fabric sample. To perform the flexibility test, the fabric sample was cut to $5 \mathrm{~cm} \times 10 \mathrm{~cm}$ pieces and sealed tightly in a polyethylene bag using a Manual Impulse Bag Sealer. The sample was then held firmly at the edge of a hard piece of wood with $3.8 \mathrm{~cm}$ of the length of the sample left outside. A $20 \mathrm{~g}$ weight was attached to the end of the sample (Figure 10) and its bending angle (BA) was measured with a protractor [10,38]. The thickness and weight of each UHMWPE fabric sample were measured before and after the nylon coating. Similarly, the thickness and weight of the UHMWPE fibre were measured before and after the nylon coating. The thickness measurement of the neat and coated fibre was done using a Renishaw Ramascope 2000 Confocal Laser Scanning Microscope. 


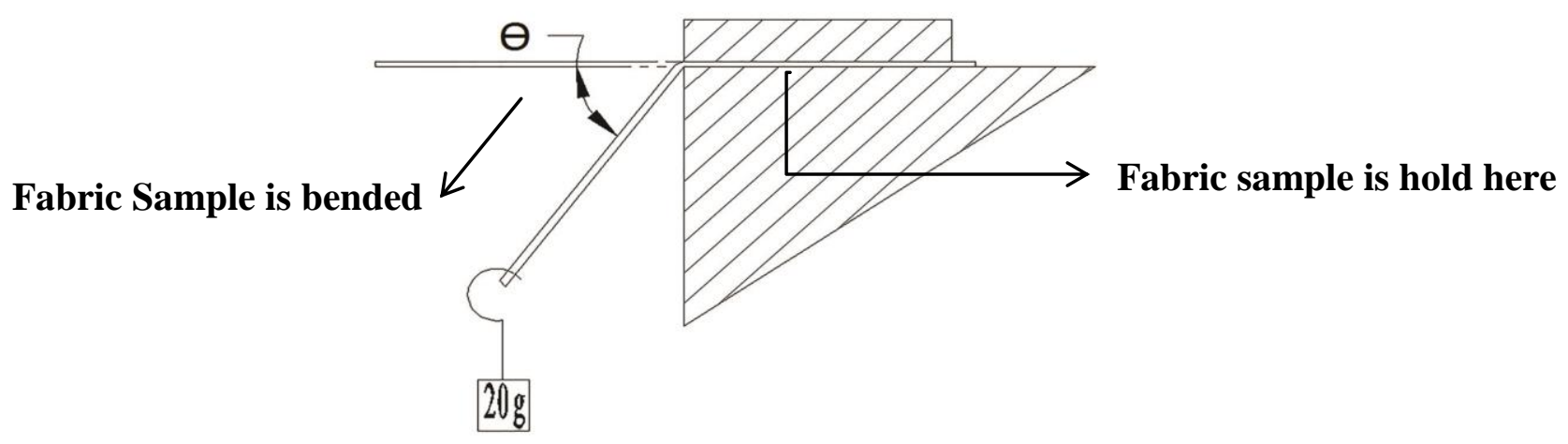

Figure 10. A schematic of the flexibility test setup for fabrics.

\section{Scanning Electron Microscopy (SEM)}

A scanning electron microscopy (SEM) analysis was carried out using a JEOL-6380LV scanning electron microscope to analyze the morphological characteristics of the nylon coating. Each sample was coated with a gold/palladium material to prevent charge buildup that could result in a poor image.

\section{Differential Scanning Calorimetry (DSC)}

The melting point of the as-received nylon pellet and converted nylon coating was measured using a Perkin Elmer Pyris Diamond differential scanning calorimeter (DSC) at the scan rate of $10 \mathrm{~K} / \mathrm{min}$. To prepare the nylon coating for the DSC analysis, nylon pellets were dissolved in the same refluxing system mentioned in section "Nylon Solution". A small amount of the nylon solution was then dropped on a wide plate and left under a vacuum chamber for two days to ensure that the alcohol was evaporated completely. The remaining thin, membrane-like nylon was immersed in a distilled water tank for $1 \mathrm{~h}$ to remove $\mathrm{CaCl}_{2}$ and then placed under vacuum for overnight to dry.

\subsubsection{Dynamic Impact Penetration Test on Fabrics}

A dynamic penetration test setup was designed similar to the standard protocols developed by the Office of Science and Technology, National Institute of Justice, United States Department of 
Justice (NIJ Standard 0115.00) [116]. A slab of backing material was prepared which a sample target was placed on top of it. The backing material pack comprised of four layers of $6.3 \mathrm{~mm}$ thick neoprene sponge, one layer of $22 \mathrm{~mm}$ thick polyethylene foam, and two layers of $6 \mathrm{~mm}$ thick dense rubber. On the other hand, the test procedure developed in this research is not fully in compliance with the NIJ Standard 0115.00 which suggests to place a single Polyart ${ }^{\mathrm{TM}}$ paper (also referred to "witness-paper") on top of the backing material pack; the depth of penetration could then be estimated by measuring the dimension of the paper cut. In a different way, five sheets of thin tracing-paper were placed on top, bottom, and between of the neoprene sponges. Consequently, the depth of penetration was estimated by counting the number of papers which were pierced by the penetrator (Table 3). The reason for such a deviation to the NIJ Standard 0115.00 was to provide an easier and more accurate measurement of the depth of penetration, which was also considered in similar research studies [3,5]. A schematic of witness-papers placed within the backing material pack is shown in Figure 11. A drop mass was machined according to the NIJ Standard consisting of a weapon and a clamping device to hold it. Three different types of stainless steel weapons were machined accordingly (Figure 12): (i) Spike which is a pointed weapon), (ii) S1, and (iii) P1 which is an edged blade; each of which weighed $450 \mathrm{~g}$. The complete assembly of such a drop mass was $2268 \mathrm{~g}$ including a threat weapon and a clamping device attached to a nylon sabot. Such a sabot was designed to ensure that the drop mass falls vertically within a drop tube apparatus in a perfect manner. The upper part of the drop mass, i.e. the sabot, was machined from a Nylon 6 rod coupled with multiple polyethylene foam discs. These discs worked as a damper to better reflect the actual motion of a human hand. The bottom part of the drop mass, i.e. the clamping holder, was made of a carbon steel pipe (Figure 13). This drop mass was released from two different arbitrary heights and falls freely under its 
own weight in a polyvinyl chloride (PVC) pipe to strike the sample target. The PVC tube was attached and fixed to a hard block wall with steel channels. The velocity of the impactor was recorded with a high speed camera (MotionPro-X3) just prior to the impact event to calculate the actual impact energy of the drop mass. The NIJ Standard 0115.00 suggests several specific impact energies (e.g. 24, 33, and $43 \mathrm{~J}$ ) to evaluate the penetration resistance of the final manufactured panel of a body armor vest which may include dozens of fabric layers. However, in this experimental study, the impact test was conducted in relatively lower impact energies to provide a more reasonable comparison between the penetration resistance of the samples with 2 or 3 layers of fabric. Such a deviation from the NIJ Standard 0115.00 assigning different impact energies was also considered in similar research studies [2,3,38].

The impact energy of the drop mass was calculated to $1.1 \mathrm{~J}$ and $2.5 \mathrm{~J}$ using the recorded impact velocity and its total mass. Consequently, for a specific impact energy, the number of ruptured witness-papers is the adopted criteria for comparing the penetration resistance of the neat and nylon coated UHMWPE fabric samples. A two layer stack of Nylon 6,6or Nylon 6,12 coated UHMWPE fabric samples, each which sealed in polyethylene bags using a manual impulse sealer, was compared individually to a three layer stack of neat UHMWPE fabric sample with equivalent areal density. The penetration depth of the impact with a specific weapon on the sample was then reported by inspecting the number of damaged witness-papers. Each test was performed in triplicate to confirm the reproducibility of the results.

Table 3. Relative depth of penetration in dynamic impact test on fabrics.

\begin{tabular}{llccccc}
\hline Number of Ruptured Witness-Paper(s) & $\mathbf{0}$ & $\mathbf{1}$ & $\mathbf{2}$ & $\mathbf{3}$ & $\mathbf{4}$ & $\mathbf{5}$ \\
\hline Approximate Penetration Depth (mm) & $\mathbf{0}$ & $\mathbf{0 - 6 . 3}$ & $\mathbf{6 . 3 - 1 2 . 6}$ & $\mathbf{1 2 . 6 - 1 8 . 9}$ & $\mathbf{1 8 . 9 - 2 5 . 2}$ & $>\mathbf{2 5 . 2}$ \\
\hline
\end{tabular}




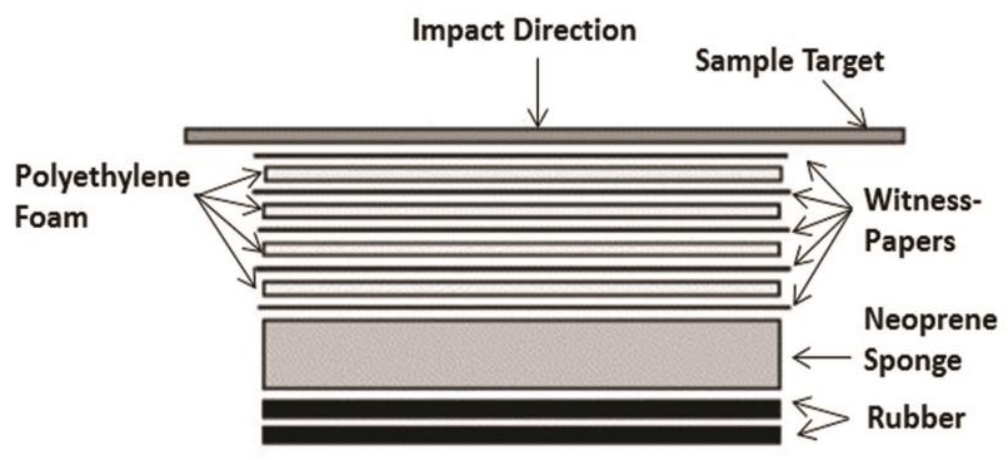

Figure 11. A schematic of the witness-papers placed within a backing material pack in dynamic penetration test setup.

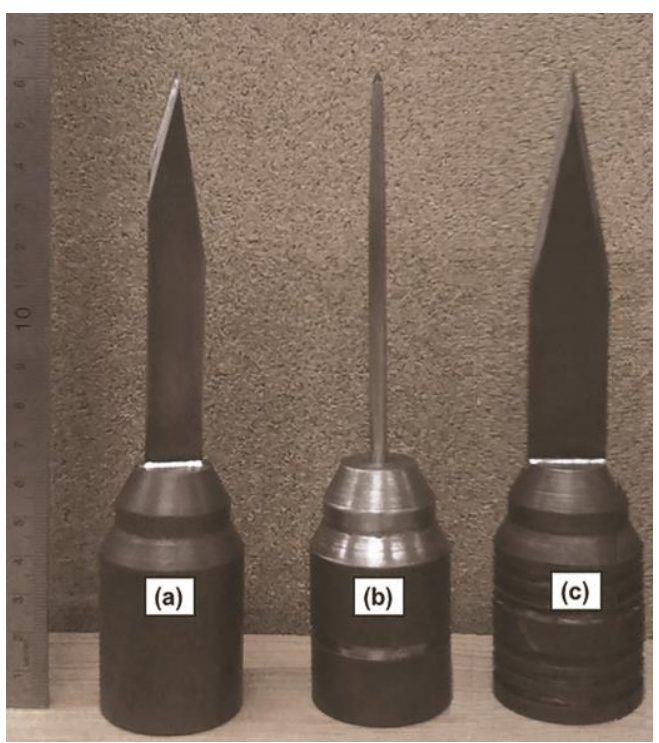

Figure 12. Weapons used for the penetration test on fabrics according to the NIJ Standard 0115.00 [116]: (a) P1 blade, (b) Spike, and (c) S1 blade.

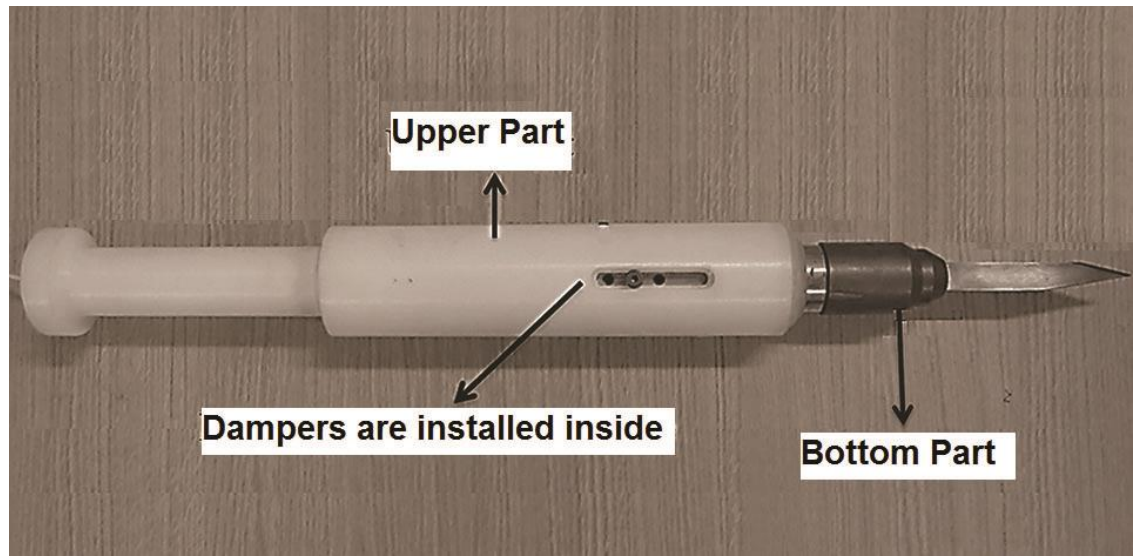

Figure 13. Drop mass weapon used for dynamic impact test on fabric samples according to the NIJ Standard 0115.00 [116]. 


\subsubsection{Quasi-Static Penetration Test On Fabrics}

A quasi-static penetration test was performed to study the mechanism of stab/puncture attack on the penetration resistance of nylon coated UHMWPE fabric sample and explore the evolution and phenomenology of the failure. A TestResources Tensile Testing Machine with a $5 \mathrm{KN}$ load cell was used for this experiment. The NIJ Standard 0115.00 weapons were mounted to the upper grip of the machine which moved through a $15 \mathrm{~cm} \times 15 \mathrm{~cm}$ sample target at $5 \mathrm{~mm} / \mathrm{min}$ for 35 $\mathrm{mm}[10,68]$.

A special fixture was machined to hold the fabric target firmly according to the ASTM D483300E1 (Figure 14) [117]. The target fabric was fixed between two concentric plates by careful tightening of three bar clamps while any induced tension was avoided. A coarse sandpaper was bonded to the opposing surface of two circular plates of the fixture to prevent fabric slippage. A two layers stack of Nylon 6,6 or Nylon 6,12 coated UHMWPE fabric was compared to a three layer stack of neat UHMWPE fabric of equivalent areal density. Each test was repeated five times to confirm its reproducibility. From the quasi-static test graph, the total puncture/stab energy dissipation of fabric samples was reported by calculating the area under the loaddisplacement curve.

Two different test conditions were applied prior to the quasi-static penetration test to assess the effect of moisture absorption on the penetration resistance of either neat and nylon coated UHMWPE fabric samples: (i) A sample was dried in vacuum chamber and stored in a sealed polyethylene bag and (ii) Another sample was fully immersed in distilled water for $24 \mathrm{~h}$. After each test on a dry sample, it was photographed on the front side to study the damage mechanism. 


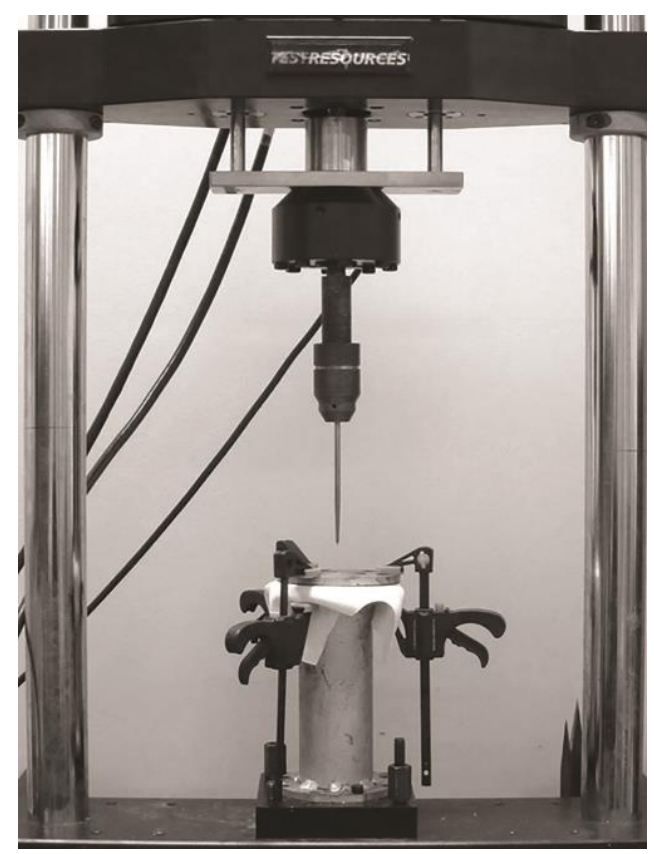

Figure 14. Quasi-static penetration test setup.

\subsubsection{Tensile and Creep Tests on Fibres}

A tensile test and a creep test were performed in a hot chamber of a SFM United Tensile Testing Machine. A special Tensile Fine Yarn Grip (TestResources Inc.) was used to perform the test with no fibre slippage (Figure 15). The tensile test was performed on each fibre sample at 25, 50, and $70{ }^{\circ} \mathrm{C}$ with the gauge length of $250 \mathrm{~mm}$ and cross speed of $2.5 \mathrm{~mm} / \mathrm{s}$ according to the ASTM D2256 [118]. The whole length of the fibre and two grips kept completely inside the hot chamber and the holes at the top and bottom of the chamber were blocked. Each test was repeated several times to obtain a reproducible result and to ensure that the fibre breakage occurs within the gauge length and not close or within the grips. The breaking force, elongation, and toughness of each fibre sample was then recorded. The creep test was performed with the same setup at a fixed load of $190 \mathrm{~N}$ at $25^{\circ} \mathrm{C}$ and $50{ }^{\circ} \mathrm{C}$, and at a fixed load of $120 \mathrm{~N}$ at $70{ }^{\circ} \mathrm{C}$ with the initial cross speed of $10 \mathrm{~mm} / \mathrm{min}$. A strain vs. time curve for the fibre sample was then produced at each temperature according to the ASTM D2990 [119]. Prior to the tensile and creep tests, each fibre sample was held for 5 min to reach thermal equilibrium condition. 


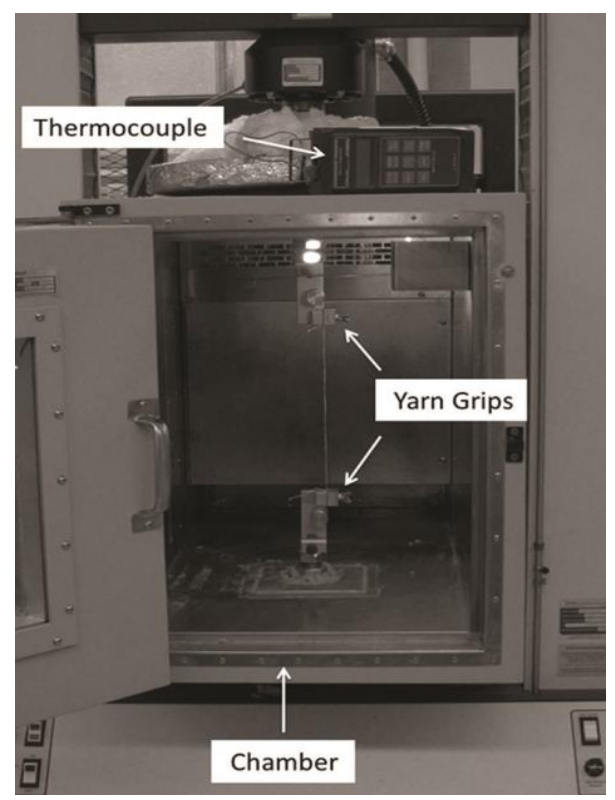

Figure 15. Tensile and creep test setup for fibres.

\subsection{Shear Thickening Fluid (STF); A Novel Approach to Fabricate a Stable STF}

\subsubsection{Material Details}

Polyethylene Glycol (PEG) was purchased from Sigma-Aldrich with an average molecular weight of $200 \mathrm{~g} / \mathrm{mol}$. The viscosity of PEG was measured to $0.05 \mathrm{~Pa}$ 's at $25{ }^{\circ} \mathrm{C}$. The hydrophilic, non-porous, and amorphous dry fumed silica was purchased from Sigma-Aldrich with the specific surface area of $200 \mathrm{~m}^{2} / \mathrm{g} \pm 25 \mathrm{~m}^{2} / \mathrm{g}$, an average primary particle size of $14 \mathrm{~nm}$, a density of $36.84 \mathrm{~kg} / \mathrm{m}^{3}$, and an estimated $3.5-4.5 \mathrm{OH} / \mathrm{m} \mu^{2}$ of the surface. The average agglomeration size of the fumed silica in its native form is $0.2-0.3 \mu \mathrm{m}$.

\subsubsection{Sample Preparation; STF Fabrication}

The fumed silica particles were dispersed gradually into PEG to a $11 \%$ loading (w/w to the total weight of the sample) and mixed thoroughly. Each sample was then treated with sequential ultrasonic irradiation and mixed thoroughly to reach either a $20 \%$ or $23 \%$ w/w using a $35 \mathrm{kHz}$ irradiation bath (VWR International). The temperature of the bath was maintained at $25{ }^{\circ} \mathrm{C}$ using a Fisher Scientific Isotemp bath circulator. At each step, it was only possible to add 
approximately $3 \% \mathrm{w} / \mathrm{w}$ particles to the dispersion, followed by a continuous ultrasonication for 5 $\mathrm{h}$ to facilitate the addition of more particles.

In the final step, the samples were ultrasonicated for $45 \mathrm{~min}$ and stirred intensively to ensure the homogeneity of the dispersion. More samples were prepared by additional ultrasonication for 45 or $90 \mathrm{~min}$. A unique designation was assumed to each sample and listed in Table 4. Finally, STF$\mathrm{C}^{*}$ was prepared for the purpose of SEM analysis by ultrasonicating STF-C for additional 315 $\min$.

Table 4. Designations of STF samples.

\begin{tabular}{lccc}
\hline Samples & $\begin{array}{c}\text { Wt. \% of Fumed } \\
\text { Silica Particles in } \\
\text { PEG }\end{array}$ & $\begin{array}{c}\text { Total Time of } \\
\text { Ultrasonication (min) }\end{array}$ & $\begin{array}{c}\text { Last Sequence of } \\
\text { Ultrasonication (min) }\end{array}$ \\
\hline STF-A & 20 & 948 & 45 \\
STF-B & 20 & 990 & 90 \\
STF-C & 20 & 1035 & 135 \\
STF-C* & 20 & 1350 & 450 \\
STF-D & 23 & 1245 & 45 \\
STF-E & 23 & 1290 & 90 \\
STF-F & 23 & 1335 & 135 \\
\hline
\end{tabular}

\subsubsection{Rheometry}

The rheological measurements were conducted using a Physica MCR-301 rheometer (AntonPaar) with parallel-plate geometry (PP25/TG) and a preferred gap size of $0.5 \mathrm{~mm}$. An AntonPaar's Peltier temperature-control hood was used to ensure virtually gradient-free sample temperature over the entire temperature range. Each sample was pre-sheared at $1 \mathrm{~s}^{-1}$ for $60 \mathrm{~s}$ to minimize the loading effect. The sample was then allowed to rest for $5 \mathrm{~min}$ before collecting data. Steady-state rotational shear flow test was performed at the shear stress ramp from $1 \mathrm{~Pa}$ to 10,000 Pa. The strain amplitude oscillatory test was performed at a fixed applied angular frequency $(\omega)$ of $1 \mathrm{rad} / \mathrm{s}$, and shear strain swept in the range of $1 \%$ to $1000 \%$. The angular frequency oscillatory test was performed at a fixed strain amplitude of $1 \%$, and angular 
frequency swept from $0.1 \mathrm{rad} / \mathrm{s}$ to $100 \mathrm{rad} / \mathrm{s}$. The flow stress was estimated from the evolution of storage $\left(\mathrm{G}^{\prime}\right)$ and loss $\left(\mathrm{G}^{\prime \prime}\right)$ moduli as a function of increasing stress amplitude from $0.1 \mathrm{~Pa}$ to $1,000 \mathrm{~Pa}$ at a constant angular frequency of $1 \mathrm{rad} / \mathrm{s}$. The temperature sweep oscillatory test was performed at a strain amplitude of $1 \%$ and a constant $\omega$ of $1 \mathrm{rad} / \mathrm{s}$ with the heating and cooling rate of $3{ }^{\circ} \mathrm{C} / \mathrm{min}$ for three cycles.

\subsubsection{Zeta Potential Measurement and SEM analysis}

A zeta potential analysis was performed using a Particle Size/Zeta Analyzer (Brookhaven Instruments 90Plus) utilizing phase analysis light scattering (PALS) technique. The measurements were performed on fumed silica dispersions in ultra pure water $(0.1 \% \mathrm{v} / \mathrm{v})$, before and after ultrasonication, similar to the acoustic technique applied in section 3.2.2.

An SEM analysis was carried out using a JEOL-6380LV scanning electron microscope. A small amount of each STF sample was placed in the oven at about $215{ }^{\circ} \mathrm{C}$ for about $5-10$ min to remove PEG. The remaining particles were coated with a gold/palladium material to prevent charge buildup. 


\section{CHAPTER 4 TEST RESULTS AND DISCUSSION}

The experimental results and the corresponding analysis of the mechanical tests and SEM analysis on the nylon coated UHMWPE fibre/fabric samples will be presented here. Initially, the analysis of the morphology of the nylon coating will be discussed from SEM micrographs, followed by the further evaluation of nylon coating using a DSC graph, followed by the study of the effect of $\mathrm{CaCl}_{2}$ on the nylon coating. Afterward, from the dynamic and quasi-static penetration test results, the performance of the neat UHMWPE fabric sample was compared to nylon coated UHMWPE fabric sample on the equivalent areal density basis, followed by the flexibility test and SEM analysis of the damage areas. Additional tensile and creep test results on the neat UHMWPE fibre and nylon coated UHMWPE fibre at three different temperatures will then be presented. In the subsequent section, the effects of ultrasound treatment in the fabrication of fumed silica dispersions in PEG will be explained from the rheological measurements, zeta potential analysis, and SEM analysis. This sequential ultrasonication technique was developed in order to develop a stable STF material with controlled shear thickening properties. Finally, the consequence of integrating this synthesized STF material with the neat and nylon coated UHMWPE fabric samples will be explained. In each of the corresponding sections, a detailed discussion of the relevant experimental results will also be presented.

\subsection{Nylon Coating; Mechanical and Penetration Test Results}

\subsubsection{Morphological Analysis and Measurements}

After immersion of the UHMWPE fibre/fabric in the methanolic nylon solution, the coated sample was subjected to an in-line water rinse to remove the residual $\mathrm{CaCl}_{2}$ salt. As a result, the nylon coating immediately contracted around the UHMWPE fibre/fabric sample forming a tight mechanical interlock. From the measurement of the neat and nylon coated UHMWPE fibre, it 
was found that they have essentially the same thickness (diameter $=0.625 \pm 0.025 \mathrm{~mm}$ ). This was likely a result of the coating shrinkage onto the UHMWPE fibre upon drying. The weight increase of fibres after nylon coating was measured to be $20 \pm 5 \%$. The SEM images presented in Figure 16 show pictures of nylon coated UHMWPE fibre at different magnification from the cross section. The images confirm that the nylon penetrates into the interior spaces of individual filaments as well as coat on the exterior surface of the fibre. From Figure 17, it was shown that the final nylon coated product is relatively uniform and stable on the UHMWPE fibre.
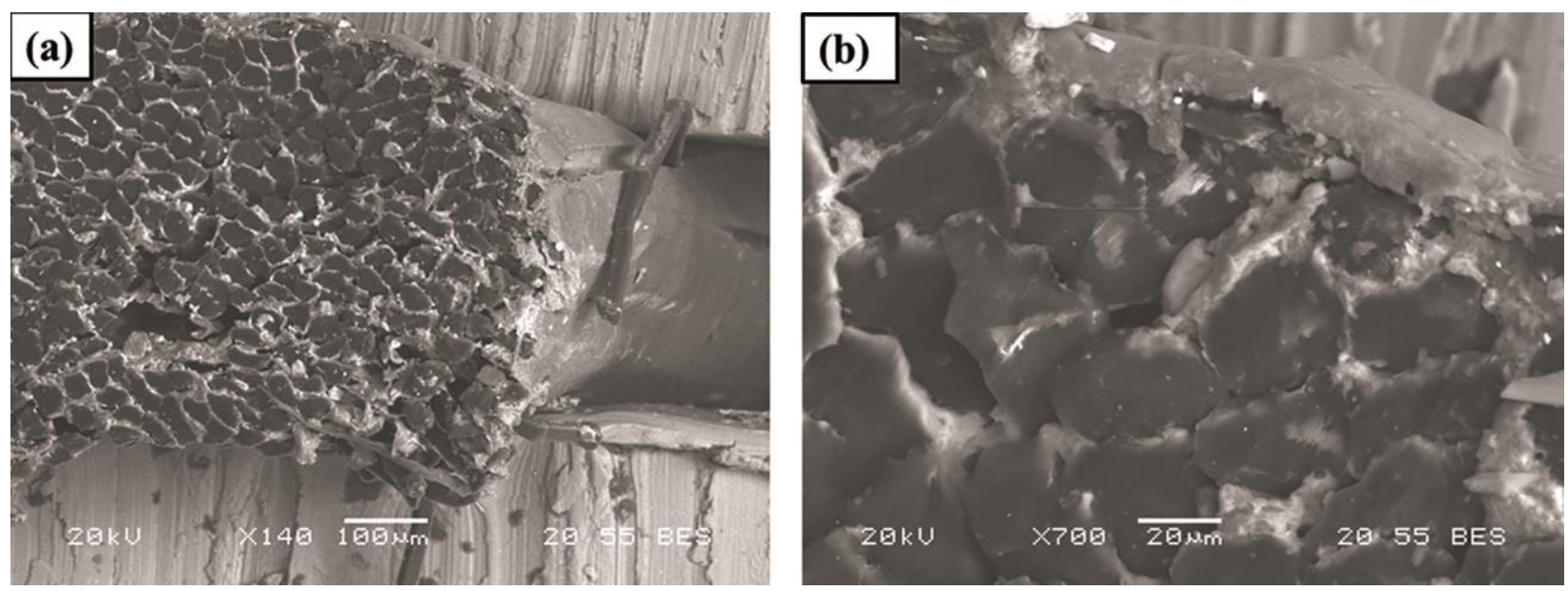

Figure 16. Cross section area of nylon coated UHMWPE fibre at different magnification (a) $\times 140$ and (b) $\times 700$.

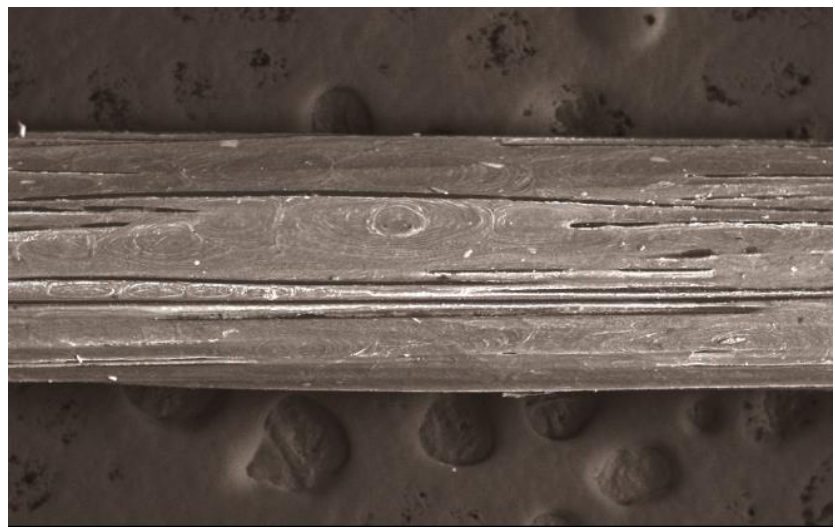

Figure 17. SEM image of the nylon coated UHMWPE fibre $(50 \times)$. 
Table 5 lists the areal density, bending angle (BA), and thickness of neat and nylon coated UHMWPE fabrics of different number of layers. The BA correlates with the flexibility of the sample; as the flexibility of the sample increases, the BA also increases. From Table 5, it was shown that one layer of neat UHMWPE fabric sample $\left(\mathrm{BA}=61 \pm 2^{\circ}\right)$ was slightly more flexible than one layer of Nylon 6,6 and Nylon 6,12 coated UHMWPE fabric samples $\left(\mathrm{BA}=54 \pm 2^{\circ}\right.$ and $55 \pm 2^{\circ}$, respectively). Also, a two layer stack of Nylon 6,6 or Nylon 6,12 coated UHMWPE fabric sample was compared to a three layer stack of neat UHMWPE fabric sample of equivalent areal density. It was found that a two layer stack of either the Nylon 6,6 coated UHMWPE fabric $\left(\mathrm{BA}=43 \pm 2^{\circ}\right)$ or Nylon 6,12 coated UHMWPE fabric $\left(\mathrm{BA}=45 \pm 2^{\circ}\right)$ was more flexible than a three layer stack of neat UHMWPE fabric $\left(\mathrm{BA}=35 \pm 1^{\circ}\right)$.

In addition, one layer of neat UHMWPE fabric sample $(0.43 \mathrm{~mm})$ was slightly thinner than one layer of Nylon 6,6 and Nylon 6,12 coated UHMWPE fabric samples $(0.48 \mathrm{~mm}$ and $0.49 \mathrm{~mm}$, respectively). Also, two layers of Nylon 6,6 and two layers of Nylon 6,12 coated fabric samples were $34 \%$ and $31 \%$ thinner (respectively) compared to three layers of a neat UHMWPE fabric sample of the same areal density basis.

Table 5. Flexibility test results on neat and nylon coated UHMWPE fabric.

\begin{tabular}{lccc}
\hline UHMWPE Fabric & $\begin{array}{c}\text { Areal Density } \\
\left(\times \mathbf{1 0}^{-\mathbf{6}} \mathbf{g} / \mathbf{c m}^{\mathbf{2}}\right)\end{array}$ & $\begin{array}{c}\text { BA } \\
(\boldsymbol{\theta})\end{array}$ & $\begin{array}{c}\text { Thickness } \\
(\mathbf{m m})\end{array}$ \\
\hline 1 Layer Neat & $231 \pm 1$ & $61 \pm 2^{\circ}$ & 0.43 \\
1 Layers Nylon 6,6 Coated & $323 \pm 8$ & $54 \pm 2^{\circ}$ & 0.47 \\
1 Layer Nylon 6,12 Coated & $323 \pm 8$ & $55 \pm 2^{\circ}$ & 0.48 \\
3 Layers Neat & $693 \pm 1$ & $35 \pm 1^{\circ}$ & 1.26 \\
2 Layers Nylon 6,6 Coated & $658 \pm 17$ & $43 \pm 2^{\circ}$ & 0.94 \\
2 Layers Nylon 6,12 Coated & $658 \pm 17$ & $45 \pm 2^{\circ}$ & 0.96 \\
\hline
\end{tabular}

SEM images for the neat and nylon-coated UHMWPE fabric samples are shown in Figures 18 from the top view. Figure 18(a) shows the top view of neat UHMWPE fabric. From Figure 18(b), it was observed that Nylon 6,6 coating penetrated and dispersed throughout the 
UHMWPE fabric filling the void spaces between the filaments. From Figure 18(c), a similar nylon penetration and coating was observed for Nylon 6,12 coated UHMWPE fabric. On the other hand, such a character could be a limitation to efficiently integrate an STF material into the fabric (see Section 4.2.7), as the voids and spaces between the filaments were covered or sealed with nylon. This limitation could be resolved for future studies with the use of an appropriate weaving machine by the coating of individual filaments prior to weave fabric. In this case, a better coating of STF materials would be expected, while the STF material could interact to a greater extent with individual filaments.
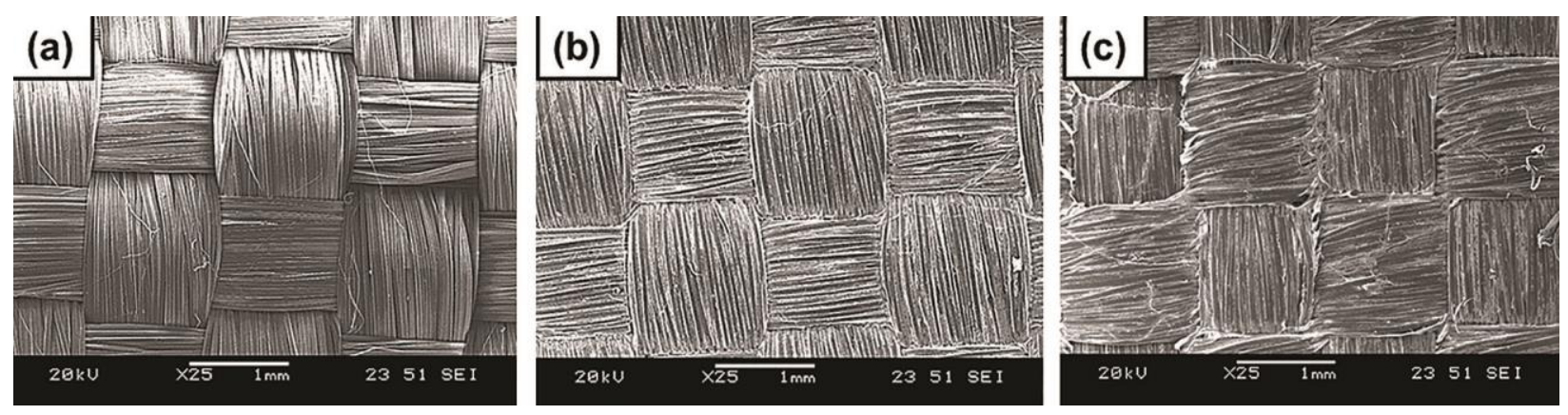

Figure 18. A magnified ( $\times 25)$ top view of fabrics: (a) Neat UHMWPE, (b) Nylon 6,6 coated UHMWPE, and (c) Nylon 6,12 coated UHMWPE.

\subsubsection{The Impact of $\mathrm{CaCl}_{2}$ on Nylon Coating}

The inter-molecular interaction/hydrogen bonding between the molecules of nylon pellets were severed when dissolved in a heated alcohol solvent containing $\mathrm{CaCl}_{2}$ salt. Here, the $\mathrm{Ca}^{2+}$ ions replaced the extensive hydrogen bonding of the amide protons of one nylon polymer chain with the carbonyl oxygens of another (or folded polymer). The role of alcohol is to facilitate such a complexation of $\mathrm{CaCl}_{2}$ with nylon (Figure 19) [120]. Alcohol also helps facilitate the wetting of individual fibre with the nylon solution. After coating, drying, and washing, some percentage of the intermolecular bonds of nylon molecules was likely restored within the system by precipitation of the residual $\mathrm{CaCl}_{2}$ salt out of the nylon with the wash water. In other words, once 
the alcohol solution containing the nylon and $\mathrm{Ca}^{2+}$ ions was exposed to water, the $\mathrm{Ca}^{2+}$ ions were removed and the hydrogen bonding restored. The results from the DSC analysis of the asreceived nylon pellets and the washed nylon coating indicated that the melting temperature of the nylon was unchanged during this process (Figure 20).

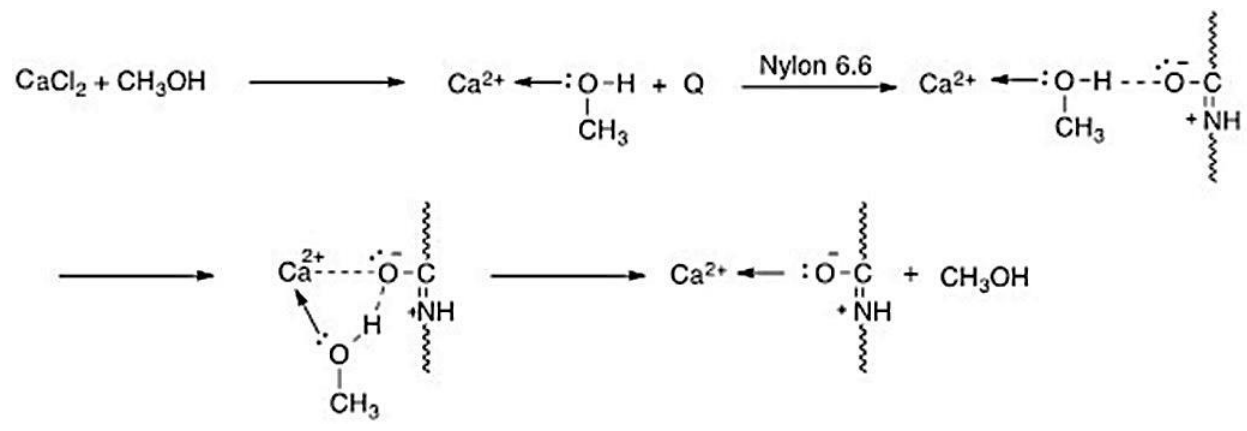

Figure 19. Chemical analysis of nylon dissolution, Adopted from [120].

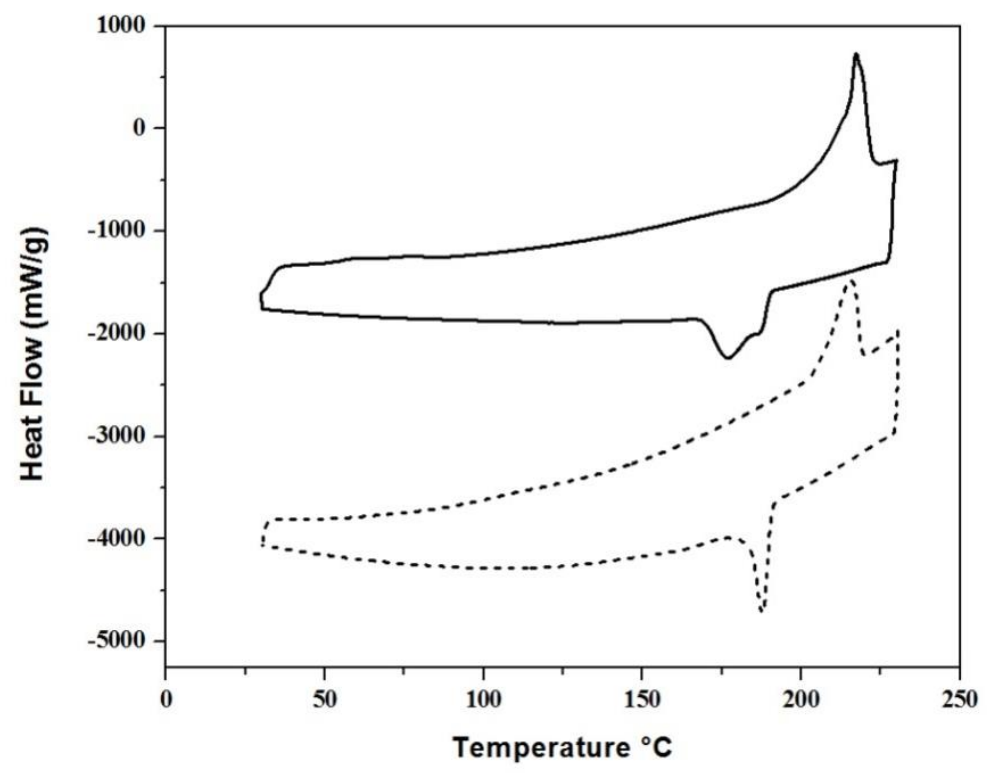

Figure 20. DSC graphs: —— as-received Nylon 6,12 pellets; ---------, Nylon 6,12 coating (refluxed and washed).

Moreover, from the SEM images of the cross-section view of samples (Figure 21), it was found that the filaments were closely packed for both nylon coated fabric samples but were loose in the neat fabric sample. This close packing was the result of mechanical interlocking of nylon coating onto the filaments after removal of $\mathrm{CaCl}_{2}$ by water washing. It was also observed from Figure 21 
that the filaments were somewhat more compact in the case of Nylon 6,12 compared to the Nylon 6,6 coated fabric. This could be interpreted to a higher restoration of hydrogen bonds and a greater reduction of void space between molecular chains upon water washing.
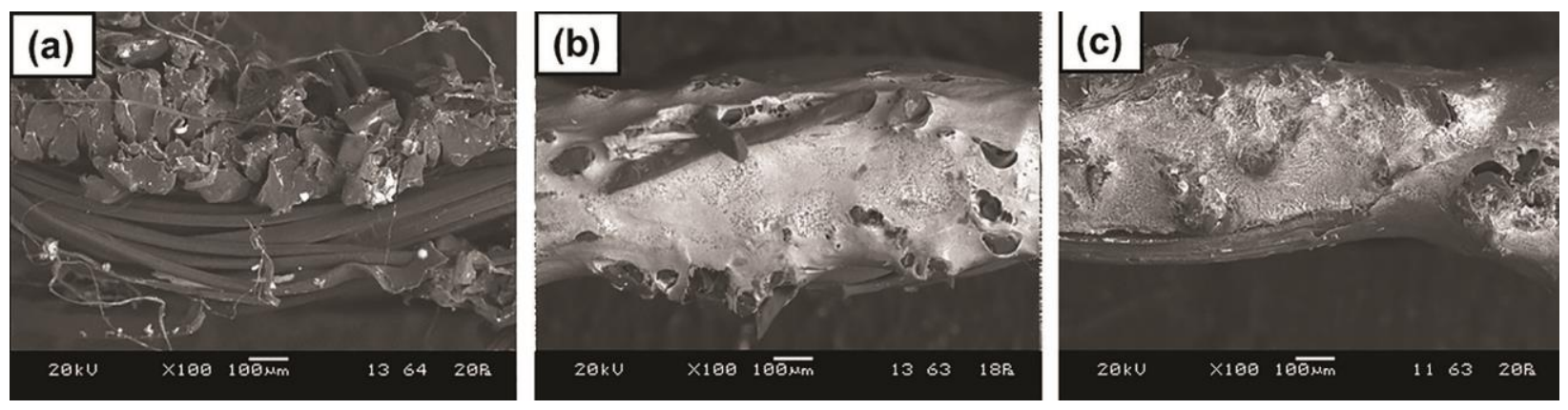

Figure 21. Cross-section view: (a) neat UHMWPE fabric, (b) Nylon 6,6 coated UHMWPE fabric, and (c) Nylon 6,12 coated UHMWPE fabric.

\subsubsection{Dynamic Impact Penetration Test Results on Fabrics}

Table 6 summarizes the results of the dynamic impact penetration test that were carried out according to the NIJ Standard 0115.00 [116]. For each impact test, a two layer stack of Nylon 6,6 or Nylon 6,12 coated UHMWPE fabric sample was compared to a three layer stack of neat UHMWPE fabric sample of equivalent areal density. At the energy level of $1.1 \mathrm{~J}$, the spike penetrated through two layers of neoprene sponges (i.e. three ruptured witness-papers) underneath the neat fabric sample, which was equal to the approximate penetration depth of 12.6-18.9 $\mathrm{mm}$ by considering the thickness of neoprene sponges presented in Table 3 . At the same energy level, only one witness-paper was ruptured with the spike underneath the Nylon 6,6 or 6,12 coated fabric sample, which was equal to the approximate depth of 0-6.3 mm (Table 3). At the energy level of $2.5 \mathrm{~J}$, the approximate depth of penetration with the spike was increased to 18.9-25.2 $\mathrm{mm}$ (i.e. four ruptured witness-papers) underneath the neat fabric sample, compared to 6.3-12.6 mm penetration (i.e. two damaged witness-papers) underneath the Nylon 6,6 or Nylon 6,12 coated fabric sample. In the case of the P1 blade, the approximate depth of penetration was 
18.9-25.2 $\mathrm{mm}$ at both $1.1 \mathrm{~J}$ and $2.5 \mathrm{~J}$ underneath the neat fabric sample; while the approximate depth of penetration was $12.6-18.9 \mathrm{~mm}$ at $1.1 \mathrm{~J}$ and $18.9-25.2 \mathrm{~mm}$ at $2.5 \mathrm{~J}$ underneath the Nylon 6,6 or Nylon 6,12 coated fabric sample. In the case of the S1 blade, the approximate depth of penetration was $12.6-18.9 \mathrm{~mm}$ at both $1.1 \mathrm{~J}$ and $18.9-25.2 \mathrm{~mm}$ at $2.5 \mathrm{~J}$ underneath the neat fabric sample; while the approximate depth of penetration was $6.3-12.6 \mathrm{~mm}$ at $1.1 \mathrm{~J}$ and $12.6-18.9 \mathrm{~mm}$ at 2.5 $\mathrm{J}$ underneath the Nylon 6,6 or Nylon 6,12 coated fabric sample.

Table 6. Dynamic impact penetration test results on fabrics for different weapons at two impact energies.

\begin{tabular}{|c|c|c|c|c|c|c|}
\hline \multirow[b]{3}{*}{ UHMWPE } & \multicolumn{6}{|c|}{ Number of Ruptured Witness-Papers } \\
\hline & \multicolumn{2}{|c|}{ Spike } & \multicolumn{2}{|c|}{ S1 Blade } & \multicolumn{2}{|c|}{ P1 Blade } \\
\hline & $1.1 \mathrm{~J}$ & $2.5 \mathrm{~J}$ & $1.1 \mathrm{~J}$ & $2.5 \mathrm{~J}$ & $1.1 \mathrm{~J}$ & $2.5 \mathrm{~J}$ \\
\hline 3 Layers of Neat & 3 & 4 & 3 & 4 & 4 & 4 \\
\hline 2 Layers of Nylon 6,6 Coated & 1 & 2 & 2 & 3 & 3 & 4 \\
\hline 2 Layers of Nylon 6,12 Coated & 1 & 2 & 2 & 3 & 3 & 4 \\
\hline
\end{tabular}

\subsubsection{Quasi-static Penetration Test Results on Fabrics}

Figures 22-24 represent the quasi-static test graphs (force versus displacement) on the neat and nylon coated UHMWPE fabric samples against the spike, S1 blade, and P1 blade. Similar to the dynamic penetration test results in Section 4.1.3, a two layers tack of Nylon 6,6 or Nylon 6,12 coated UHMWPE fabric sample was compared to a three layer stack of neat UHMWPE fabric sample of equivalent areal density. From Figure 22, it was observed that two a layer stack of Nylon 6,6 coated UHMWPE fabric sample and a two layer stack of Nylon 6,12 coated UHMWPE fabric sample provided $77 \%$ and $86 \%$ improvement (respectively) of the maximum resistance force compared to a three layer stack of neat UHMWPE fabric sample. In all the cases, a maximum resistance peak force was detected around 8-9 $\mathrm{mm}$ of the penetration distance 
through the sample. An acoustic burst sound heard at the maximum breaking force may correspond to the local breakage of several close fibres at the same time. After the peak force, the load dropped suddenly as the spike's tip was driven completely through the sample. It was concluded that the fibres, which were pushed aside by the penetrator, could still exert force on the spike as the load increase was then interpreted to the presence of frictional force between such failed fibres and the spike penetrator.

From Figure 23, it was observed that either a two layer stack of Nylon 6,6 or Nylon 6,12 coated UHMWPE fabric sample provided superior cut resistance against an S1 blade compared to a three layer stack of neat UHMWPE fabric sample. From Figure 23, it was also observed that the improvement of the cut resistance of fabric samples is more noticeable up to about the first 20 mm of penetration. According to the NIJ Standard 0115.00, a higher penetration resistance is the highest priority up to this point, as vital organs of a human body could get seriously damaged by a blade-shape weapon afterwards [116]. From Figure 24, it was observed that either a two layer stack of Nylon 6,6 or Nylon 6,12 coated UHMWPE fabric sample provided almost the same cut resistance against a P1 blade as three layers stack of neat UHMWPE fabric sample, although, a slight improvement was noticed for the first $10 \mathrm{~mm}$ of penetration in the case of the nylon coated fabric samples. It was also observed from Figures 23 and Figure 24 that the amplitude of oscillation of graphs for the neat and nylon coated UHMWPE fabric samples were large and irregular which likely corresponded to the cutting or motion of several bunches of filaments at the same time, while an apparent peak was not detected from the load-deflection curves whereas it was observed in Figure 22. Instead, the load increased monotonically with increasing the penetration depth. The reason for such a behaviour is the shape of the blade's cutting edge, 
which cuts the filament continuously through the fabric and results in a more localized damage. Hence, the restriction in fibre motion became less significant.

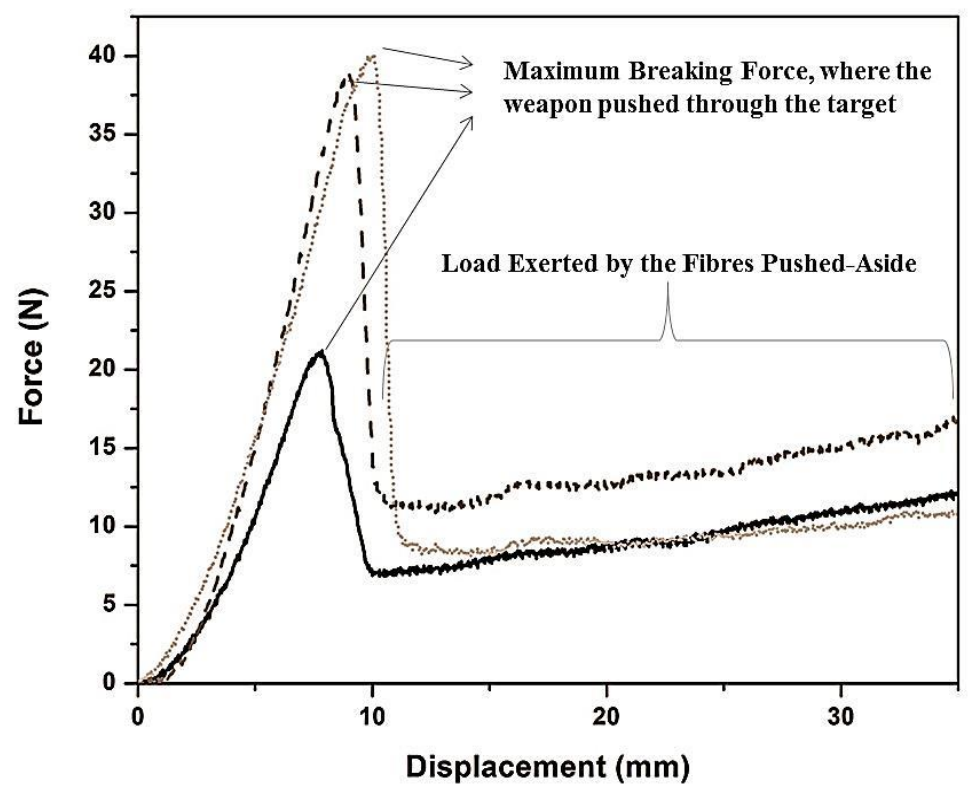

Figure 22. Force versus displacement graphs from the quasi-static penetration test results with a spike on: — , 3 layer stack of neat UHMWPE fabric; --------, 2 layer stack of Nylon 6,6 coated UHMWPE fabric; ........, 2 layer stack of Nylon 6,12 coated UHMWPE fabric.

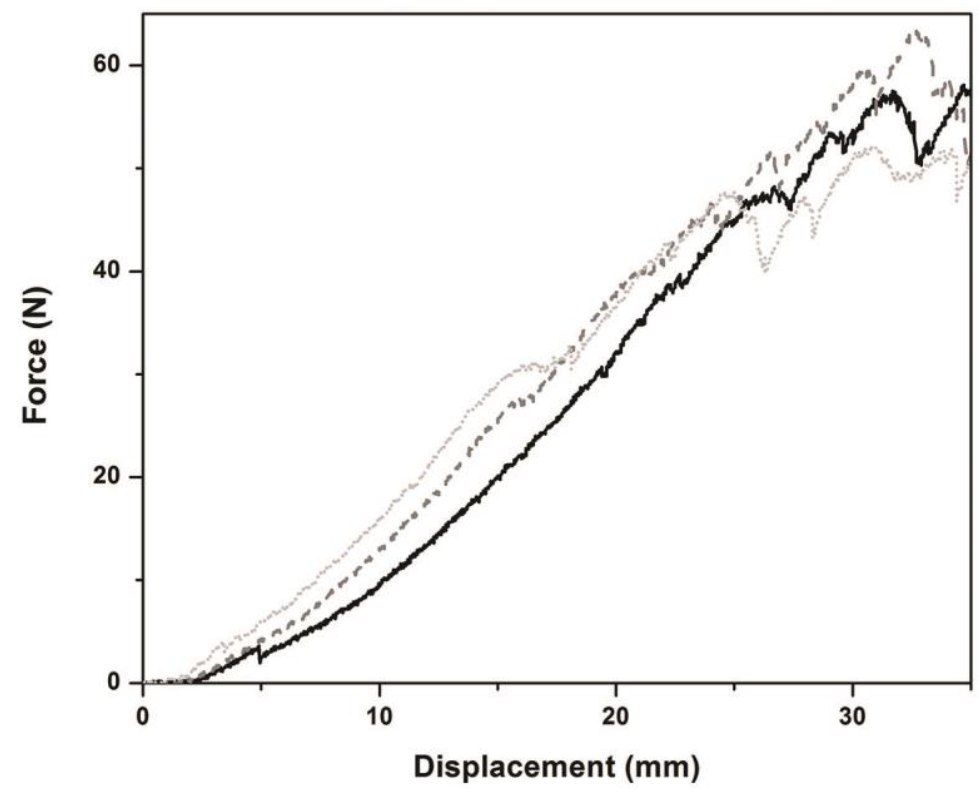

Figure 23. Force versus displacement graphs from the quasi-static penetration test results with an S1 blade on: — , 3 layer stack of neat UHMWPE fabric; ---------, 2 layer stack of Nylon 6,6 coated UHMWPE fabric;........, 2 layer stack of Nylon 6,12 coated UHMWPE fabric. 


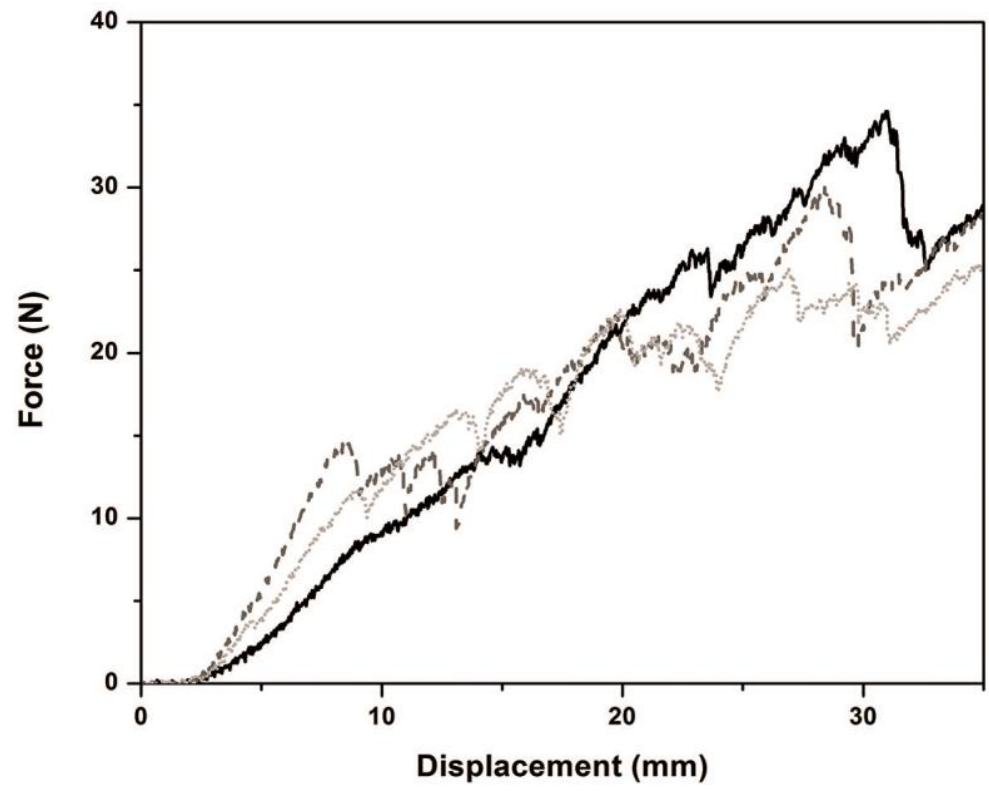

Figure 24. Force versus displacement graphs from the quasi-static penetration test results with aP1 blade on: - 3 layer stack of neat UHMWPE fabric; --------, 2 layer stack of Nylon 6,6 coated UHMWPE fabric; ......., 2 layer stack of Nylon 6,12 coated UHMWPE fabric.

Consequently, the energy absorption values of sample fabrics against each weapon, i.e. the work done by the penetrator was calculated from the area under load-deflection curves and listed in Table 7. Against the spike, the energy absorption value for a three layer stack of neat UHMWPE fabric sample, a two layer stack of Nylon 6,6 coated UHMWPE fabric sample, and a two layer stack of Nylon 6,12 coated UHMWPE fabric sample were calculated to $0.580 \mathrm{~J}, 0.603 \mathrm{~J}$, and $0.550 \mathrm{~J}$, respectively.

Against the S1 blade, the energy absorption value for a three layer stack of neat UHMWPE fabric sample, a two layer stack of Nylon 6,6 coated UHMWPE fabric sample, and a two layer stack of Nylon 6,12 coated UHMWPE fabric sample were calculated to $0.937 \mathrm{~J}, 0.975 \mathrm{~J}$, and $0.992 \mathrm{~J}$, respectively. Against the P1 blade, the energy absorption value for a three layer stack of neat UHMWPE fabric sample, a two layer stack of Nylon 6,6 coated UHMWPE fabric sample, and a two layer stack of Nylon 6,12 coated UHMWPE fabric sample were calculated to $0.580 \mathrm{~J}$, $0.603 \mathrm{~J}$, and $0.550 \mathrm{~J}$, respectively. 
Table 7. Energy dissipation in quasi-static penetration test for different weapons on a 2 or 3 layer stack of fabric.

\begin{tabular}{lccc}
\hline & \multicolumn{2}{c}{ The energy absorbed by fabric samples $\left(\times \mathbf{1 0}^{-3} \mathbf{J}\right)$} \\
\cline { 2 - 4 } UHMWPE & Spike & S1 Blade & P1 Blade \\
\hline 3 layers neat & $295 \pm 27$ & $937 \pm 83$ & $580 \pm 21$ \\
2 layers of Nylon $\mathbf{6 , 6}$ coated & $455 \pm 37$ & $975 \pm 75$ & $603 \pm 12$ \\
2 layers of Nylon $\mathbf{6 , 1 2}$ coated & $427 \pm 23$ & $992 \pm 85$ & $550 \pm 31$ \\
\hline
\end{tabular}

More tests were performed on a single fabric layer of neat and nylon coated UHMWPE fabric (graphs are not provided). From the calculated energy values, it was also observed that a single layer of Nylon 6,6 or Nylon 6,12 coated fabric sample provides superior resistance than a single layer of the neat fabric sample against all three types of weapons (Table 8). The effect of water absorption on a single layer of neat and nylon coated fabric was also studied. According to the calculated energy absorption values listed in Table 8, there was no significant change reported in the quasi-static test results of the neat and nylon coated fabric samples under two different test conditions as mentioned in Section "Investigating the effect of moisture absorption".

It was also confirmed that there was no irreversible weight gain by the neat or nylon coated fabric sample by immersing in water. Moreover, we observed that the increase in the weight fraction of $\mathrm{CaCl}_{2}$ in the refluxing system did not positively impact the quasi-static penetration test results. Comparing the results in Section 3.1.4 and 3.1.5, it was noticed that the dynamic test results were in a good agreement with the quasi-static test results for both the neat and nylon coated UHMWPE fabric samples. 
Table 8. Energy dissipation in quasi-static penetration test for different weapons on a single layer fabric.

\begin{tabular}{|c|c|c|c|c|c|c|}
\hline \multirow[b]{3}{*}{ UHMWPE } & \multicolumn{6}{|c|}{ The Energy Absorbed by Fabric Samples $\left(\times 10^{-3} \mathrm{~J}\right)$} \\
\hline & \multicolumn{2}{|c|}{ Spike } & \multicolumn{2}{|c|}{ S1 Blade } & \multicolumn{2}{|c|}{ P1 Blade } \\
\hline & Dry & Wet & Dry & Wet & Dry & Wet \\
\hline 1 Layer Neat & $73 \pm 07$ & $66 \pm 06$ & $405 \pm 15$ & $325 \pm 24$ & $199 \pm 15$ & $176 \pm 15$ \\
\hline 1 Layer of Nylon 6,6 Coated & $211 \pm 23$ & $198 \pm 14$ & $692 \pm 25$ & $676 \pm 25$ & $292 \pm 22$ & $280 \pm 16$ \\
\hline 1 Layer of Nylon 6,12 Coated & $231 \pm 13$ & $220 \pm 17$ & $693 \pm 21$ & $665 \pm 31$ & $332+25$ & $322 \pm 11$ \\
\hline
\end{tabular}

\section{Fabric Damage Mechanisms in Quasi-static Penetration Test}

Figures 25(a), 26(a), and 27(a) show the top view of the neat and nylon coated UHMWPE fabric targets after the quasi-static penetration test with a spike. It was shown in Figure 25(a) that the neat fibres were more loose and pushed aside (separated) noticeably by the spike penetrator when compared to nylon coated UHMWPE fabric samples depicted in Figure 26(a) and 27(a), where the coated fibres were more closely packed. In other words, the spike penetrator could separate neat fibres more easily by pushing them aside while the spike faced less resistance from the fabric sample. Although the windowing effect (i.e. pushing the fibres aside by the penetrator instead of cutting them) was the dominant failure mechanism in the penetration test using a spike weapon for all samples, it was less significant in the case of nylon coated fabric samples. Evidence of fibre breakage was not clearly observed from the SEM images, although, an acoustic burst sound heard at the maximum breaking force may correspond to the local breakage of several close fibres at the same time. Overall, the higher penetration resistance of nylon coated fabric could be explained as the larger area of the coated fabric was involved in such a penetration because of increased fibre-fibre and fibre-spike frictional forces and the fibres engaged together more efficiently. Figures 25(b,c), 26(b,c), and 27(b,c), the top view of the neat and nylon coated UHMWPE fabric targets after the quasi-static penetration test with knife-like 
blade, confirm the previous observation in the SEM images from the spike threat: More loose fibres were found in the case of the neat fabric sample, while the fibres became more closepacked when coated with nylon. Figures $25(\mathrm{~b}, \mathrm{c})$ showed more evidence of fibre breakage (or cuts) in the case of neat UHMWPE fabric sample compared to nylon coated UHMWPE fabric samples. It was also observed that a blade threat resulted in a more localized damage by cutting the fibres along the blade edge and the restriction in fibre motion became less significant; opposite to the situation for the puncture test. Hence, a more localized damage could be the key reason why nylon coated samples showed more improvement over uncoated samples against a spike weapon, but not against S1 and P1 blades when the cutting mechanism dominated windowing effects.
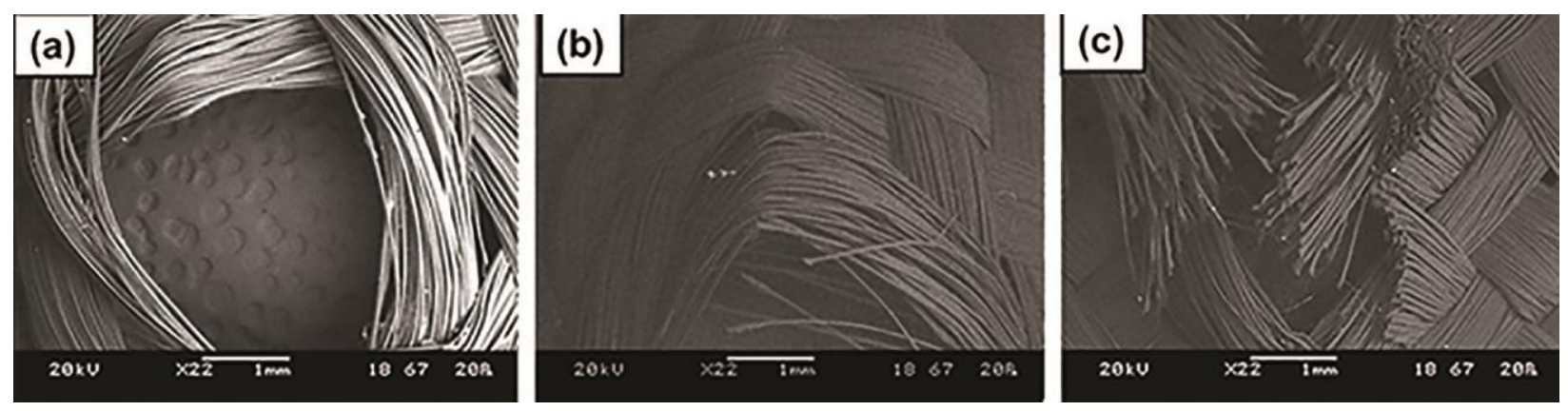

Figure 25. SEM images of the ruptured area after quasi-static penetration test on neat UHMWPE fabric using: (a) Spike; (b) S1 blade; (c) P1 blade.
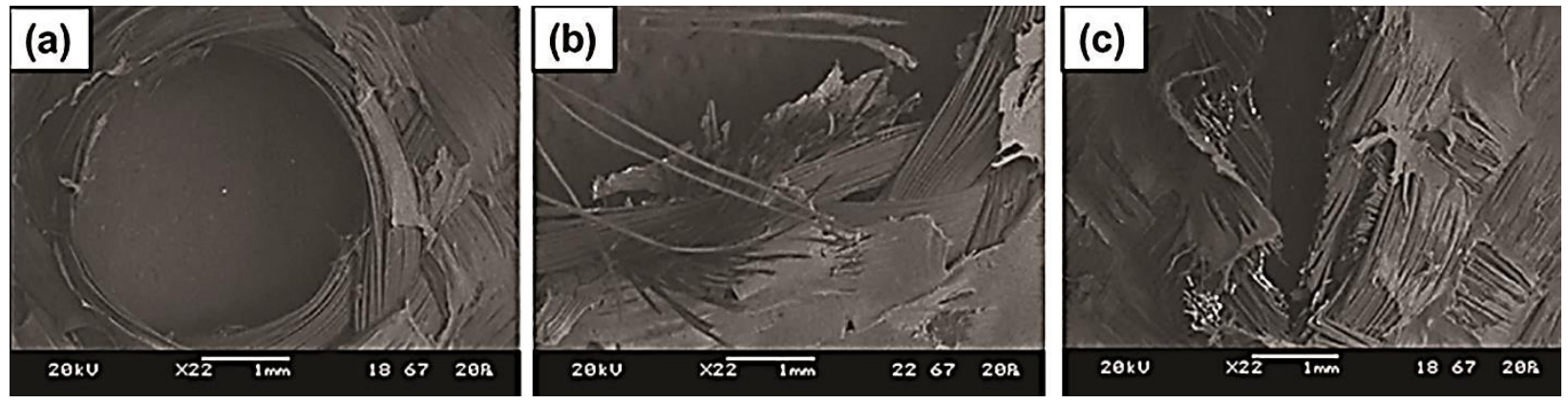

Figure 26. SEM images of the ruptured area after quasi-static penetration test on Nylon 6,12 coated UHMWPE fabric using: (a) Spike; (b) S1 blade; (c) P1 blade. 

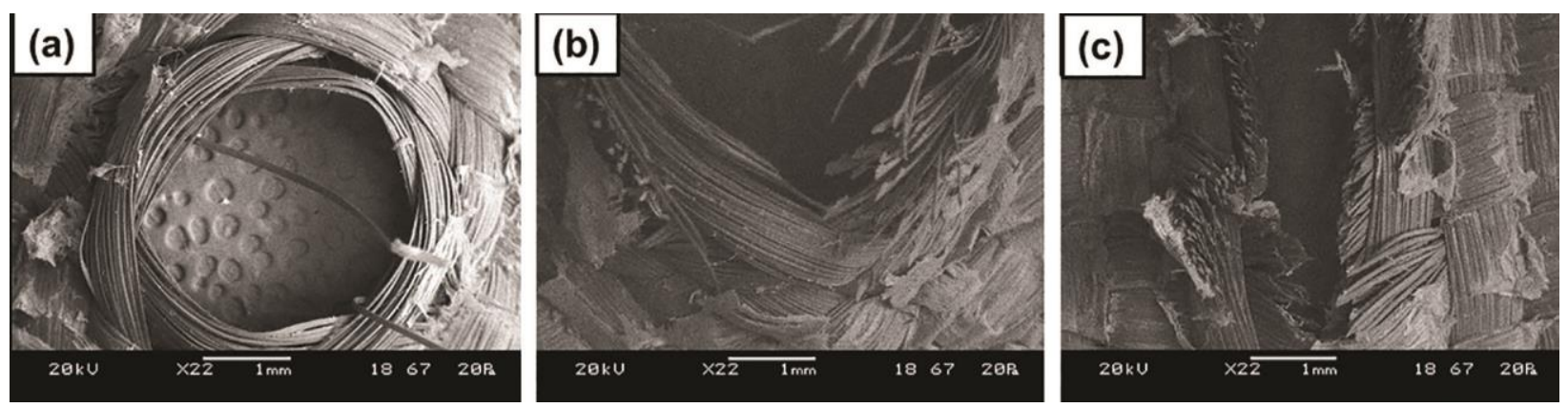

Figure 27.SEM images of the ruptured area after quasi-static penetration test on Nylon 6,12 coated UHMWPE fabric using: (a) Spike; (b) S1 blade; (c) P1 blade.

From Table 8, it was observed that the effect of water absorption is minimal in the case of nylon coated UHMWPE fabric samples compared with the neat UHMWPE fabric after immersing the samples in water for $24 \mathrm{~h}$ (see Section 3.1.5). As a result, the energy absorption of neat, Nylon 6,6 coated, and Nylon 6,12 coated UHMWPE fabric samples was reduced by 17, 5, and 6\% (respectively) against the spike weapon; 24, 2, and 4\% (respectively) against the S1 blade; and 13, 4, and 3\% (respectively) against the P1 blade. On the other hand, UHMWPE's water absorption is less than $0.01 \%$ (only about $1 \%$ that of Nylon 6,6 ). Although, it was concluded that moisture behaves more like a lubricant on the neat fabric sample and decreases the penetration resistance of the fabric as the weapon could push the fibres aside easier in penetration.

\subsubsection{Tensile/Creep Test Results on Fibres}

Figures 28-30 represent the tensile test graphs (force versus displacement) on the neat and nylon coated UHMWPE fabric samples at 25,50 , and $70{ }^{\circ} \mathrm{C}$. From the graphs, the maximum breaking force and elongation of each fibre sample were listed in Table 9. Also, the toughness value of each fibre sample was calculated by measuring the area under the stress-strain curve and listed in Table 9.

From the three graphs, it was observed that nylon coated UHMWPE fibre provided superior tensile strength compared to the neat UHMWPE fibre at 25,50 , and $70{ }^{\circ} \mathrm{C}$. It was observed that 
Nylon 6,6 and Nylon 6,12 coated fibres showed 17\% and 16\% improvement (respectively) in the maximum breaking force at $25^{\circ} \mathrm{C}$. At $50{ }^{\circ} \mathrm{C}$ and $70{ }^{\circ} \mathrm{C}$, the improvement in maximum breaking strength is reported as the following: $14 \%$ and $6 \%$ in the case of Nylon 6,6 coated UHMWPE fibre (respectively) and $15 \%$ and $2 \%$ in the case of Nylon 6,12 coated UHMWPE fibre (respectively), which were listed in Table 9. From the elongation values listed in Table 9, it was observed that the elongation of nylon coated fibres from tension was comparable with the neat fibre confirming the flexibility and ductile nature of the nylon coating onto the UHMWPE fibre. From the toughness values listed in Table 9, it was observed that nylon coated fibres showed higher toughness compared to the neat fibre with the average of $32 \%$.

The increase in toughness values of nylon coated fibre compared to the neat one is more significant at high temperatures: the average increase of $54 \%$ and $46 \%$ at $50{ }^{\circ} \mathrm{C}$ and $70{ }^{\circ} \mathrm{C}$, respectively. From the elastic portion of the stress-strain curve, the elastic modulus was calculated for the neat and nylon coated UHMWPE fibre at $25{ }^{\circ} \mathrm{C}$. It was found that the elastic modulus of Nylon 6,6 and Nylon 6,12 coated fibres dropped by approximately 19\% and 30\% (respectively) compared to the neat fibre which was $20.6 \pm 0.7 \mathrm{GPa}$. 


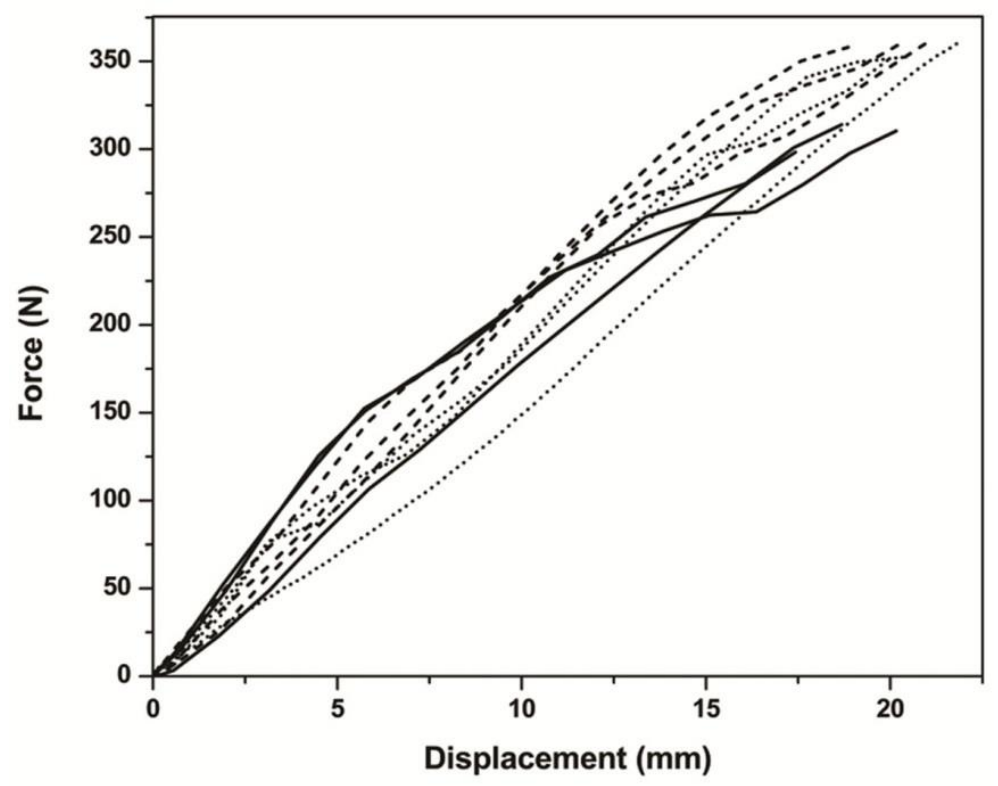

Figure 28. Force versus displacement graphs from the tensile test at $25^{\circ} \mathrm{C}$ : neat UHMWPE fibre; --------, Nylon 6,6 coated UHMWPE fibre; , Nylon 6,12 coated UHMWPE fibre (gauge length of $250 \mathrm{~mm}$ and cross speed of $2.5 \mathrm{~mm} / \mathrm{s}$ ).

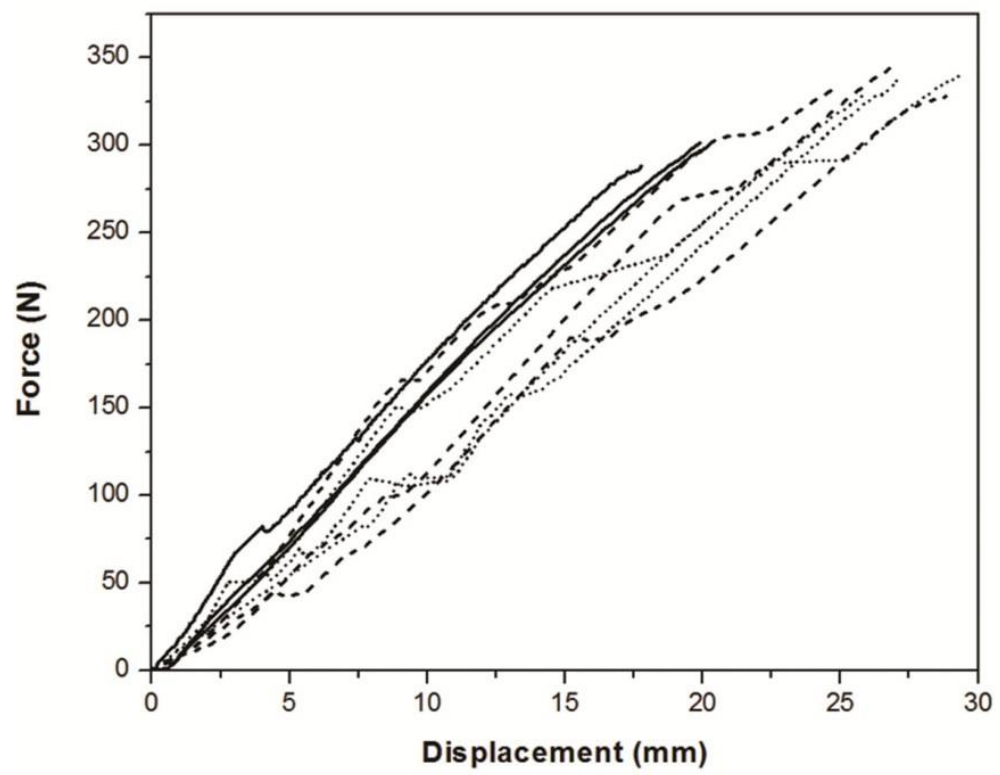

Figure 29. Force versus displacement graphs from the tensile test at $50{ }^{\circ} \mathrm{C}$ : neat UHMWPE fibre; --------, Nylon 6,6 coated UHMWPE fibre; Nylon 6,12 coated UHMWPE fibre (gauge length of $250 \mathrm{~mm}$ and cross speed of $2.5 \mathrm{~mm} / \mathrm{s}$ ). 


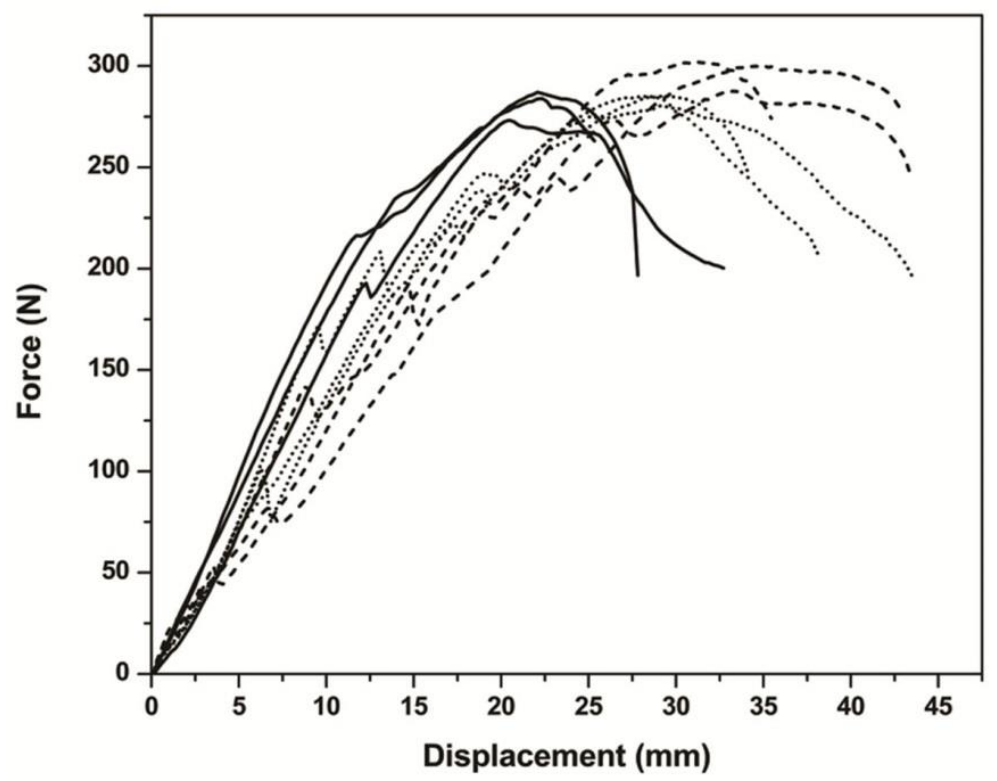

Figure 30. Force versus displacement graphs from the tensile test at $70{ }^{\circ} \mathrm{C}:-$, neat UHMWPE fibre; ---------, Nylon 6,6 coated UHMWPE fibre; ........, Nylon 6,12 coated UHMWPE fibre (gauge length of $250 \mathrm{~mm}$ and cross speed of $2.5 \mathrm{~mm} / \mathrm{s}$ ).

Table 9. Tensile properties of neat and nylon coated UHMWPE fibres at 25,50 , and $70{ }^{\circ} \mathrm{C}$.

\begin{tabular}{|c|c|c|c|c|c|c|c|c|c|}
\hline \multirow[b]{2}{*}{$\begin{array}{l}\text { UHMWPE } \\
\text { Fibre }\end{array}$} & \multicolumn{3}{|c|}{$\mathrm{T}=25^{\circ} \mathrm{C}$} & \multicolumn{3}{|c|}{$\mathbf{T}=50^{\circ} \mathrm{C}$} & \multicolumn{3}{|c|}{$\mathrm{T}=70^{\circ} \mathrm{C}$} \\
\hline & $\begin{array}{c}\text { Max. } \\
\text { Breaking } \\
\text { Force }(\mathbf{N})\end{array}$ & $\begin{array}{l}\text { Elongation } \\
\text { Percentage }\end{array}$ & $\begin{array}{l}\text { Toughness } \\
\left(\times 10^{6} \mathrm{~J} / \mathrm{m}^{3}\right)\end{array}$ & $\begin{array}{c}\text { Max. } \\
\text { Breaking } \\
\text { Force }(\mathrm{N})\end{array}$ & $\begin{array}{l}\text { Elongation } \\
\text { Percentage }\end{array}$ & $\begin{array}{l}\text { Toughness } \\
\left(\times 10^{6} \mathrm{~J} / \mathrm{m}^{3}\right)\end{array}$ & $\begin{array}{c}\text { Max. } \\
\text { Breaking } \\
\text { Force }(\mathbf{N})\end{array}$ & $\begin{array}{l}\text { Elongation } \\
\text { Percentage }\end{array}$ & $\begin{array}{l}\text { Toughness } \\
\left(\times 10^{6} \mathrm{~J} / \mathrm{m}^{3}\right)\end{array}$ \\
\hline Neat & $307 \pm 8$ & $7.5 \pm 0.5$ & $38.8 \pm 5.6$ & $292 \pm 3$ & $7.7 \pm 0.5$ & $39.7 \pm 2.4$ & $280 \pm 6$ & $11.4 \pm 5.3$ & $70 \pm 0.8$ \\
\hline $\begin{array}{l}\text { Nylon 6,6 } \\
\text { coated }\end{array}$ & $360 \pm 5$ & $8.0 \pm 0.5$ & $52.7 \pm 4.6$ & $334 \pm 8$ & $10.4 \pm 0.5$ & $60.4 \pm 0.4$ & $298 \pm 6$ & $16.5 \pm 1.7$ & $105 \pm 1.3$ \\
\hline $\begin{array}{l}\text { Nylon 6,12 } \\
\text { coated }\end{array}$ & $355 \pm 4$ & $8.3 \pm 0.4$ & $50.4 \pm 1.4$ & $336 \pm 6$ & $11.0 \pm 0.7$ & $61.9 \pm 5.1$ & $285 \pm 3$ & $16.2 \pm 3.1$ & $99 \pm 1.2$ \\
\hline
\end{tabular}

*(gauge length of $250 \mathrm{~mm}$ and cross speed of $2.5 \mathrm{~mm} / \mathrm{s}$ ).

Figures 31-33 represent the creep test graphs (strain versus time) on the neat and nylon coated UHMWPE fabric samples at 25, 50, and $70^{\circ} \mathrm{C}$. From the graphs, it was observed that Nylon 6,6 and Nylon 6,12 coated UHMWPE fibres showed 27\% and 14\% improvement (respectively) in creep resistance at $25^{\circ} \mathrm{C}$. At $50{ }^{\circ} \mathrm{C}$ and $70{ }^{\circ} \mathrm{C}$, the improvement in creep resistance is reported as the following: $34 \%$ and 19\% in the case of Nylon 6,6 and Nylon 6,12 coated UHMWPE fibre (respectively) and listed in Table 10 and 11. In general, Nylon 6,6 coated UHMWPE fibre could 
provide better creep resistance at ambient and elevated temperatures compared with Nylon 6,12 coated UHMWPE fibre.

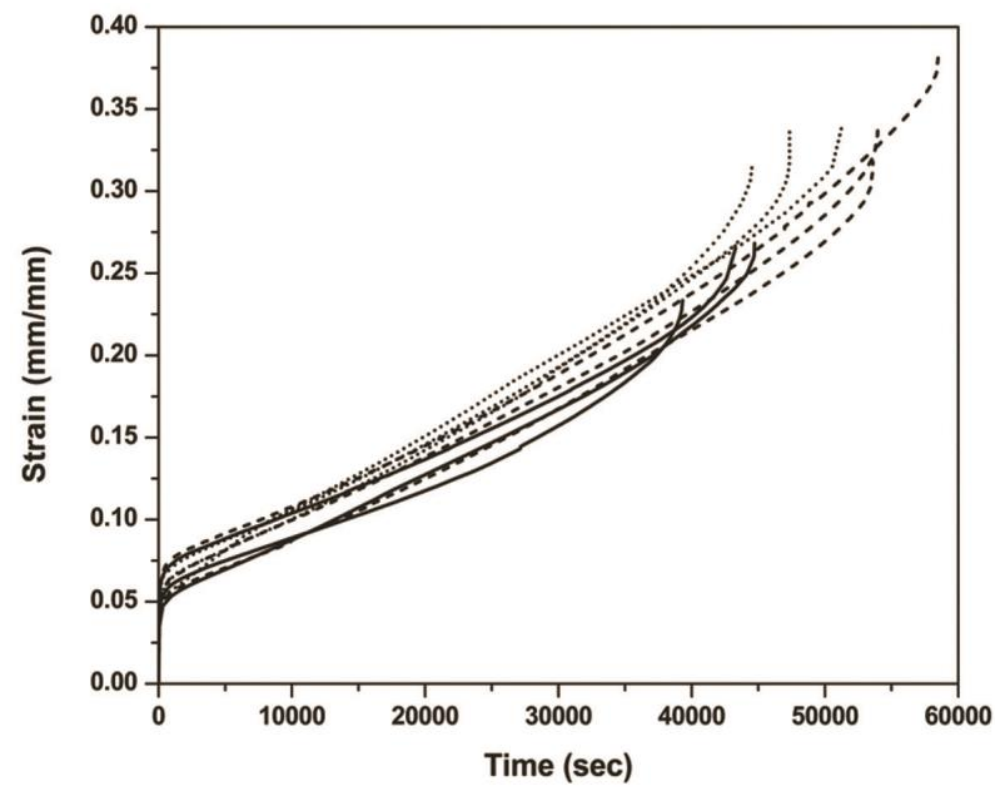

Figure 31. Strain versus time graphs from the creep test at a fixed load of $190 \mathrm{~N}$ at $25{ }^{\circ} \mathrm{C}$ : — UHMWPE fibre; ---------, Nylon 6,6 coated UHMWPE fibre; ........, Nylon 6,12 coated UHMWPE fibre (gauge length of $250 \mathrm{~mm}$ ).

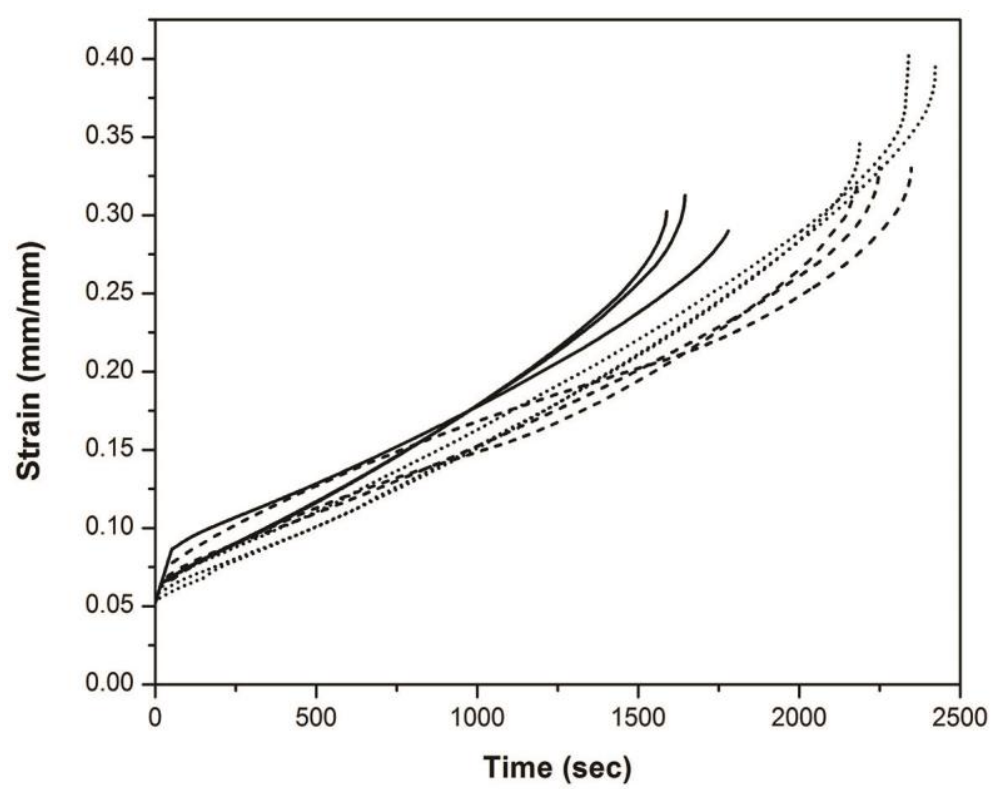

Figure 32. Strain versus time graphs from the creep test at a fixed load of $190 \mathrm{~N}$ at $50{ }^{\circ} \mathrm{C}$ : — UHMWPE fibre; ---------, Nylon 6,6 coated UHMWPE fibre; ........, Nylon 6,12 coated UHMWPE fibre (gauge length of $250 \mathrm{~mm}$ ). 


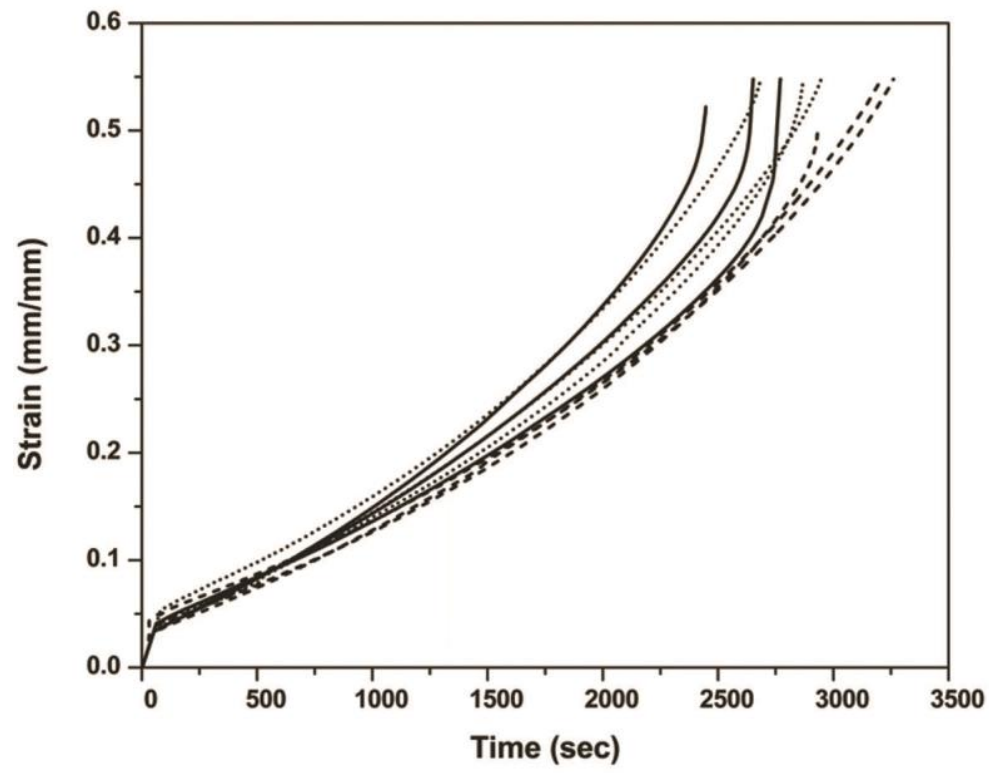

Figure 33. Strain versus time graphs from the creep test at a fixed load of $120 \mathrm{~N}$ at $70{ }^{\circ} \mathrm{C}$ : — UHMWPE fibre; ---------, Nylon 6,6 coated UHMWPE fibre; ........., Nylon 6,12 coated UHMWPE fibre (gauge length of $250 \mathrm{~mm}$ ).

Table 10. Maximum creep time of neat and nylon coated UHMWPE fibres at 25 and $50^{\circ} \mathrm{C}$.

\begin{tabular}{|c|c|c|c|}
\hline & $\mathrm{T}=25^{\circ} \mathrm{C}$ & $\mathbf{T}=50^{\circ} \mathbf{C}$ & $\mathbf{T}=70^{\circ} \mathrm{C}$ \\
\hline UHMWPE Fibre & $\begin{array}{c}\text { Max. Creep } \\
\text { Time (s) }\end{array}$ & $\begin{array}{c}\text { Max. Creep } \\
\text { Time (s) }\end{array}$ & $\begin{array}{c}\text { Max. Creep } \\
\text { Time (s) }\end{array}$ \\
\hline Neat & $41963 \pm 5 \%$ & $1751 \pm 6 \%$ & $2623 \pm 6 \%$ \\
\hline Nylon 6,6 Coated & $55344 \pm 5 \%$ & $2340 \pm 4 \%$ & $3134 \pm 6 \%$ \\
\hline Nylon 6,12 Coated & $47747 \pm 7 \%$ & $2397 \pm 5 \%$ & $2835 \pm 5 \%$ \\
\hline
\end{tabular}

*At a fixed load of $190 \mathrm{~N}$ (Gauge length of $250 \mathrm{~mm}$ ).

Table 11. Maximum creep time of neat and nylon coated UHMWPE fibres at $70{ }^{\circ} \mathrm{C}$.

\begin{tabular}{cc}
\hline UHMWPE Fibre & \begin{tabular}{c}
$\mathbf{T}=\mathbf{7 0}{ }^{\circ} \mathbf{C}$ \\
\cline { 2 - 2 } Max. Creep \\
Time (s)
\end{tabular} \\
\hline Neat & $2623 \pm 6 \%$ \\
Nylon 6,6 Coated & $3134 \pm 6 \%$ \\
Nylon 6,12 Coated & $2835 \pm 5 \%$
\end{tabular}

*At a fixed load of $120 \mathrm{~N}$ (Gauge length of $250 \mathrm{~mm}$ ). 


\section{Fibre Damage Mechanisms in Tension/Creep}

Figure 34(a) was taken from a broken Nylon 6,6 coated UHMWPE fibre in tension at $25{ }^{\circ} \mathrm{C}$. From Figure 34(a), substantial breakage was detected on the nylon coating of the broken fibre, while small segments of the coating were were remained on the filaments close to the rupture point. Figure 34(b) was taken from a Nylon 6,6 coated UHMWPE fibre prior to the progressive breakage of filaments in tension. From Figure 34(b), fibre breakage or massive coating rupture was not detected, while several small size rips were detected on the nylon coating. It is plausible to conclude that the nylon coating started to break at various local sites prior to the breakage of the filaments. A similar morphological observation was also obtained for Nylon 6,12 coated UHMWPE fibre in tension.
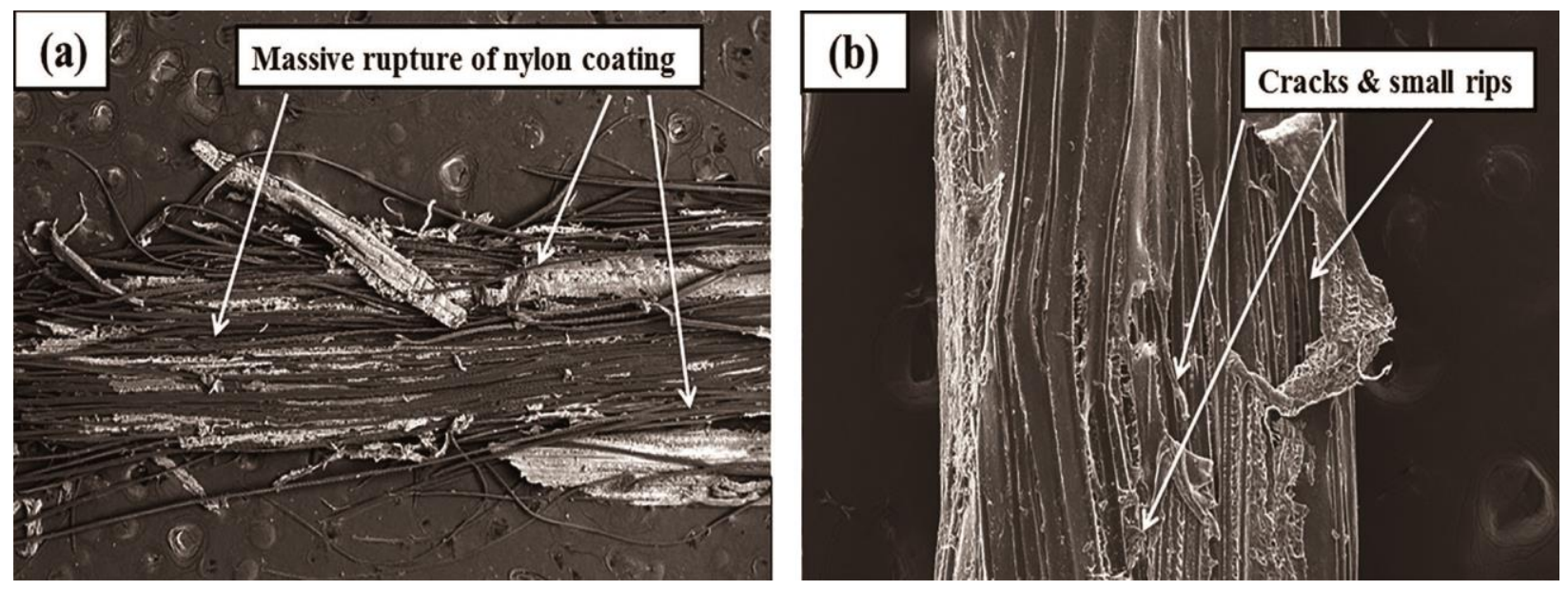

Figure 34. (a) SEM image of the ruptured area of a Nylon 6,6 coated UHMWPE fibre from tension at 25 ${ }^{\circ} \mathrm{C}(20 \times)$; (b) SEM image of a Nylon 6,6 coated UHMWPE fibre prior to the progressive breakage of filaments in tension at $25^{\circ} \mathrm{C}(60 \times)$.

Figure 35 was taken from a broken Nylon 6,6 coated UHMWPE fibre in creep test at $25{ }^{\circ} \mathrm{C}$. From Figure 35, multiple small crazes were detected on the nylon coating of the broken fibre. These crazes were initiated on the surface of nylon coating and propagated most likely prior to the breaking of individual filaments. The filaments then fractured progressively until the entire fibre broke at the latest stage of the creep test. Also, the evidence of flat and serrated ruptured 
surfaces of nylon coating could demonstrate a ductile-brittle failure mechanism. A similar morphological observation was also obtained for Nylon 6,12 coated UHMWPE fibre in creep test.

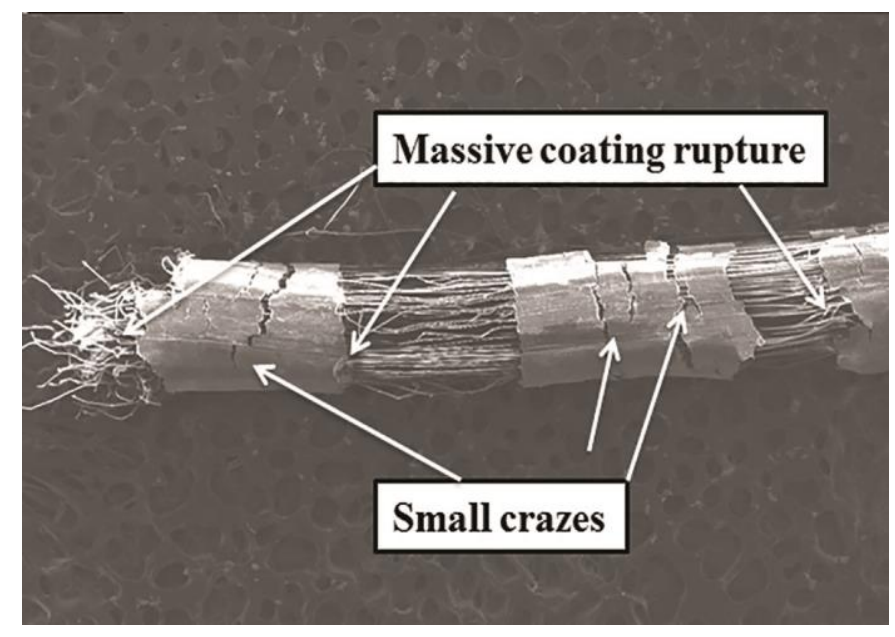

Figure 35. SEM image of the ruptured area of a Nylon 6,6 coated UHMWPE fibre from creep test at 25 ${ }^{\circ} \mathrm{C}(15 \times)$.

\subsubsection{Summary}

The mechanical interlocking of Nylon 6,6 and Nylon 6,12 could create a uniform and stable coating on the inherently inert UHMWPE fibre/fabric as a result of nylon film shrinkage upon removal of $\mathrm{CaCl}_{2}$ and drying. From the penetration test results on fabric samples, a two layer stack of Nylon 6,6 or 6,12 coated UHMWPE fabric sample demonstrated higher puncture resistance compared to a three layer stack of neat UHMWPE fabric sample of an equal areal density with no loss of flexibility and a reduced total thickness requirement, while affording the same level of stab resistance. In the results, the maneuverability and mobility of the worn security clothing could be improved with such a cost effective, thin, and flexible alternative to comparable body armor systems.

A single layer of nylon coated sample demonstrated superior resistance against both puncture and cut threats regardless of the consequent weight increase. Consequently, we propose that 
nylon coated UHMWPE could be a framework that facilitates extensive hydrogen bonding between amide linkages and other materials, like Kevlar ${ }^{\circledR}$, and may be compatible with shear thickening fluid (STF) materials.

An attempt to investigate the use of nylon coatings on UHMWPE to improve its interaction with STF materials will be discussed in Section 4.2.7. In general, it was demonstrated that the nylon coated UHMWPE fibre had higher tensile strength, higher creep resistance, lower elastic modulus, and higher toughness than the neat UHMWPE fibre. In addition, SEM images were analyzed to study the failure mechanism of fabric samples under the penetration test and fibre samples under tensile or creep testing (see Section 4.1.4 and 4.1.5).

\subsection{STFs; Rheological Measurements and Morphological Analysis}

\subsubsection{Rotational Steady-State Rheometry}

Figures 36 represent the steady-state rheological measurement in shear stress controlled mode showing apparent viscosity $(\eta)$ as a function of increasing shear rate for different STF samples which were described in Table 4. For each STF sample, a noticeable shear thinning trend at a low shear rate was detected while viscosity reached to a plateau followed by a discontinuous transition to shear thickening region by further increases in shear rate. From the literature, for fumed silica dispersions, it was explained that weak steric repulsive force and Brownian motion prevent further association of fumed silica aggregates at very low shear rates [73]. The viscosity of the suspension decreased by increasing shear rate because some of the agglomerations were disrupted and the orientation of fumed silica particles aligns with the direction of the shear flow $[10,73,121]$.

In general, this early shear thinning region could be indicative of a non-flocculated structured colloidal system [10,73], and represents a sign of yielding [10]. In general, the discontinuous 
shear thickening phenomenon in fumed silica dispersions was interpreted to the domination of hydrodynamic interactions over colloidal forces [73,101], meaning that the restoring effect of Brownian motion could no longer withstand the strength of applied hydrodynamic forces [73,104]. In other words, in a concentrated silica dispersion, long-range hydrodynamic forces push the particles from a repulsive dominated region to a closer average interparticle distance where strong attractive forces were dominant $[73,101]$. Finally, by the formation of fumed silica aggregates, the viscosity of the dispersion increased abruptly [73].

After the maximum shear thickened regime, the viscosity graph dropped sharply. This behaviour was interpreted to the collapse of the three dimensional transient shear thickened structure or possibly it was just because of simple slip of the solidified sample against the tooling [10].

Another explanation is that this sharp drop of the viscosity graph is a second shear thinning region after the collapse of the structural network, $[122,123]$ as there would be a possibility that these aggregates could come to an orderly state at very large deformations [73]. The onset of shear thickening, i.e. the critical shear rate $\left(\dot{\gamma}_{c}\right)$, and maximum shear thickened viscosity $\left(\eta_{\max }\right)$ for different STF samples are presented in Table 12. 


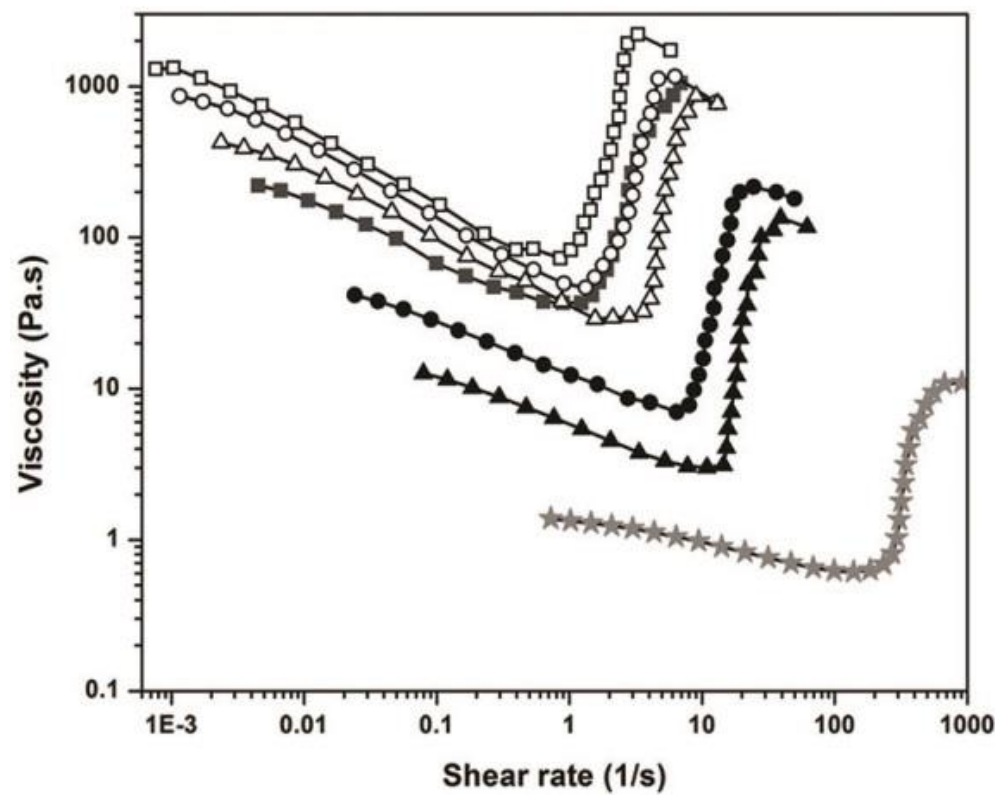

Figure 36. Apparent viscosity as a function of shear rate at different times of ultrasonication (at the last stage of fabrication process) and weight fractions of fumed silica particles in PEG (steady-state shear stress controlled): $\mathbf{\square}$, (STF-A), 20\% w/w, $45 \mathrm{~min}$; , (STF-B), 20\% w/w, $45 \mathrm{~min}$; $\boldsymbol{\Delta}$, (STF-C), 20\% w/w, $135 \mathrm{~min}$; $\star$, (STF-C*), 20\% w/w, $450 \mathrm{~min}$; $\square$, (STF-D), 23\% w/w, $45 \mathrm{~min}$; O, (STF-E), 23\% w/w, $45 \mathrm{~min} ; \triangle$, (STF-F), $23 \% \mathrm{w} / \mathrm{w}, 135 \mathrm{~min}$.

Table 12. Critical shear rate and maximum shear thickened viscosity for steady-shear stress controlled rheometry.

\begin{tabular}{lccccccc}
\hline \multicolumn{1}{c}{ Sample } & STF-A & STF-B & STF-C & STF-C* & STF-D & STF-E & STF-F \\
\hline$\dot{\boldsymbol{\gamma}}_{\boldsymbol{c}}\left(\mathbf{s}^{-1}\right)$ & 1.2 & 8 & 14 & 220 & 0.5 & 1.5 & 2.1 \\
$\boldsymbol{\eta}_{\max }(\mathbf{P a} \cdot \mathbf{s})$ & 1050 & 250 & 135 & 12 & 2200 & 1160 & 860 \\
\hline
\end{tabular}

\subsubsection{Flow Stress}

The apparent flow stress of the STF sample was estimated from the dynamic oscillation mode at the crossover point of $\mathrm{G}^{\prime}$ and $\mathrm{G}^{\prime \prime}$ moduli which demonstrates a complete transition from an elastically dominated region to a viscous dominated region. At the flow point, the internal structure of the sample breaks to such an extent that the material can flow [124].

Figure 37 depicts the changes in oscillating dynamic moduli as a function of increasing shear stress at constant frequency of $1 \mathrm{rad} / \mathrm{s}$ for STF-A, STF-B, and STF-C. All the samples contained $20 \% \mathrm{w} / \mathrm{w}$ of fumed silica particles in PEG with different levels of ultrasonication as STF- 
$\mathrm{A}<\mathrm{STF}-\mathrm{B}<\mathrm{STF}-\mathrm{C}$. The flow stress of STF-A and STF-B was then identified at $12 \mathrm{~Pa}$ and $4 \mathrm{~Pa}$, respectively, while STF-C did not exhibit a crossover point which illustrates that its flow point was not detectable. Figure 38 depicts the changes in oscillating dynamic moduli as a function of increasing shear stress at constant frequency of $1 \mathrm{rad} / \mathrm{s}$ for STF-D, STF-E, and STF-F. All the samples contained $23 \% \mathrm{w} / \mathrm{w}$ of fumed silica particles in PEG with different levels of ultrasonication: STF-D $<$ STF-E $<$ STF-F. The flow stress of STF-D, STF-E, and STF-F was then identified at $30 \mathrm{~Pa}, 22 \mathrm{~Pa}$, and $16 \mathrm{~Pa}$, respectively.

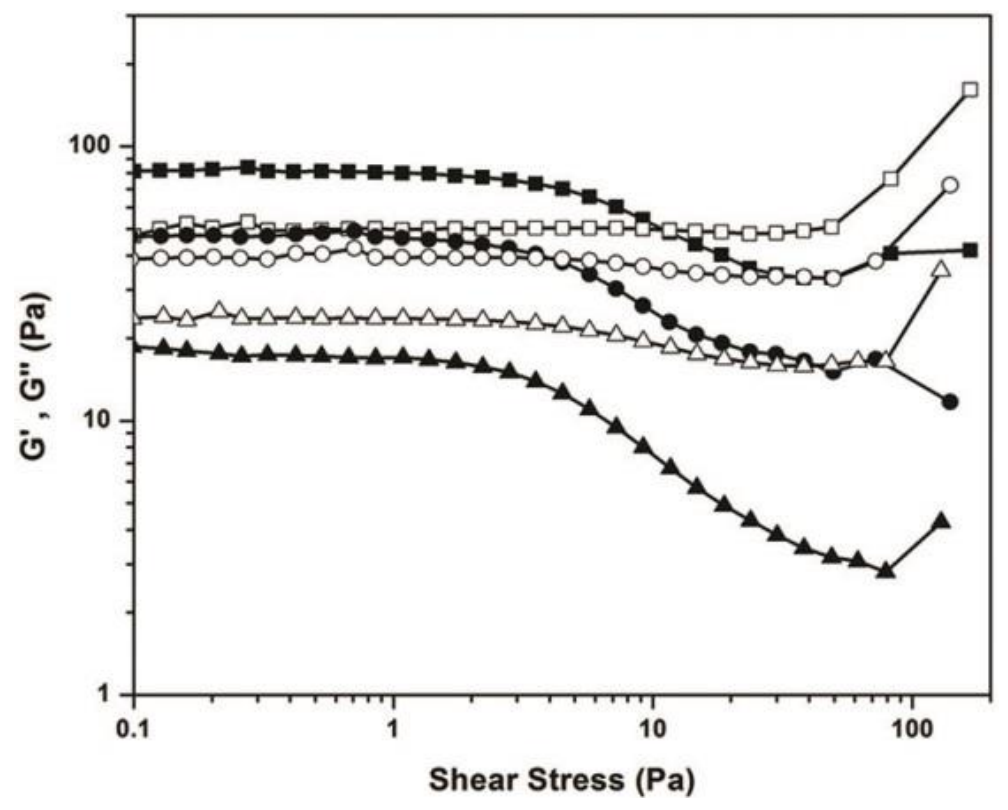

Figure 37. Storage and loss modulus as a function of shear stress ( $\mathrm{and} \omega=1 \mathrm{rad} / \mathrm{s}$ ) at different times of ultrasonication (at the last stage of fabrication process) and the same concentration of fumed silica particles in PEG (20\% w/w): $\mathbf{\square}, \square$, (STF-A), $45 \mathrm{~min} ; \boldsymbol{\bullet}, \mathrm{O},(\mathrm{STF}-\mathrm{B}), 45 \mathrm{~min} ; \boldsymbol{\Delta}, \triangle,(\mathrm{STF}-\mathrm{C}), 135$ min; filled symbols: G'; empty symbols: G', 


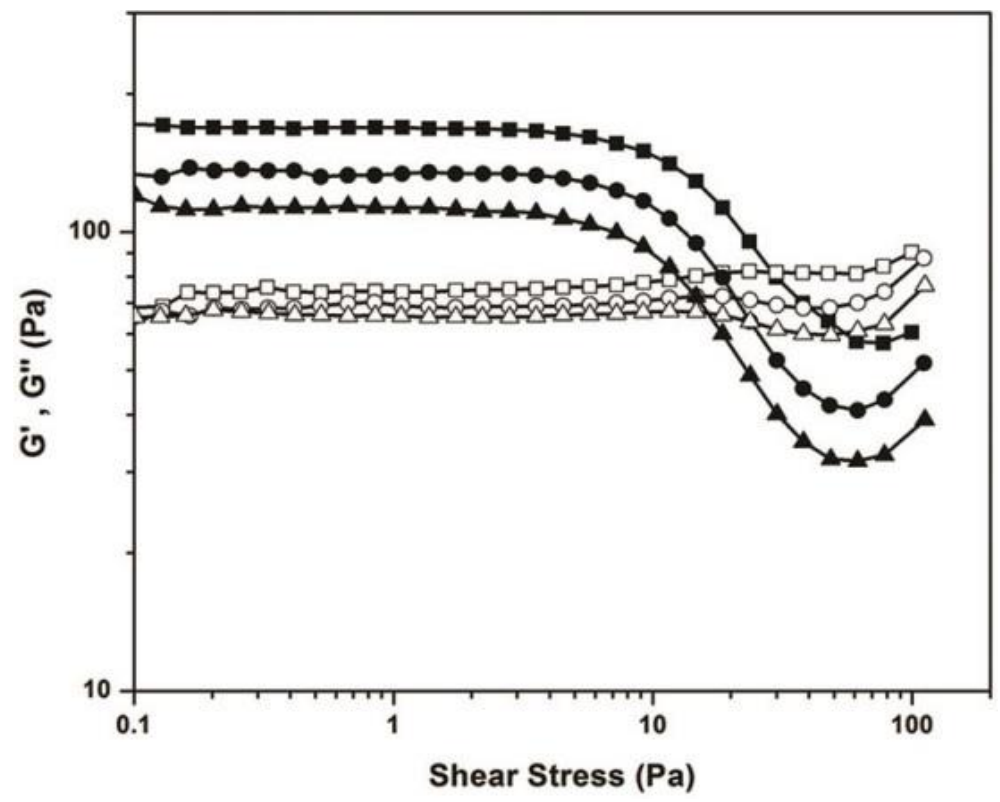

Figure 38. Storage and loss modulus as a function of shear stress (and $\omega=1 \mathrm{rad} / \mathrm{s}$ ) at different times of ultrasonication (at the last stage of fabrication process) and the same concentration of fumed silica particles in PEG (23\% w/w): $\boldsymbol{\square}, \square$, (STF-D), $45 \mathrm{~min}$; $\bullet$, $\bigcirc$, (STF-E), $45 \mathrm{~min} ; \boldsymbol{\Delta}, \triangle$, (STF-F), $135 \mathrm{~min}$; filled symbols: G'; empty symbols: G'.

\subsubsection{Dynamic Rheometry}

The response of STF samples at low and high strain amplitudes was studied at a constant angular frequency of $1 \mathrm{rad} / \mathrm{s}$. Figure 39 depicts the changes in oscillating dynamic moduli as a function of increasing imposed strain amplitude for STF-A, STF-B, and STF-C. Each of the graphs exhibited a constant plateau (i.e. linear viscoelastic behaviour, LVE) at a small or a moderate shear strain amplitudes. According to the literature, such a small strain LVE behaviour contributes to the ability of Brownian motion to restore the structure of the system to an equilibrium state, therefore, G' remains constant [100]. In the case of STF-A, G' exceeded G" at small and moderate strain amplitudes, and a crossover point of G' and G'' was detected at the strain of $11 \%$ which defines a transition flow point $\left(\gamma_{c}\right)$ for this material. Consequently, STF-A exhibited a kind of rigidity or gel-like behaviour before $\gamma_{c}$, but showed a liquid-like behaviour after $\gamma_{c}$. For STF-B and STF-C, G" exceeded G' at all strain amplitudes and a crossover point of $\mathrm{G}^{\prime}$ and $\mathrm{G}^{\prime \prime}$ was not detected which shows the viscous-like characteristic of both samples. 
Additionally, the moduli started to jump to higher values at $\gamma=160 \%$ in the case of STF-A, while $\mathrm{G}^{\prime \prime}$ remained higher than $\mathrm{G}^{\prime}$.

From the literature, in general, G" domination over $G^{\prime}$ is considered as a sign of non-flocculated suspensions $[73,125]$. An abrupt jump of the moduli could also be a sign of strain hardening in dynamic oscillation, as both shear rate and shear stress increased drastically when $G^{\prime}$ and $G^{\prime \prime}$ jumped to a higher level $[73,84]$. Eventually, both moduli dropped sharply at a very large strain in the case of STF-A. This behaviour could be interpreted to the total breakdown of its colloidal structure.

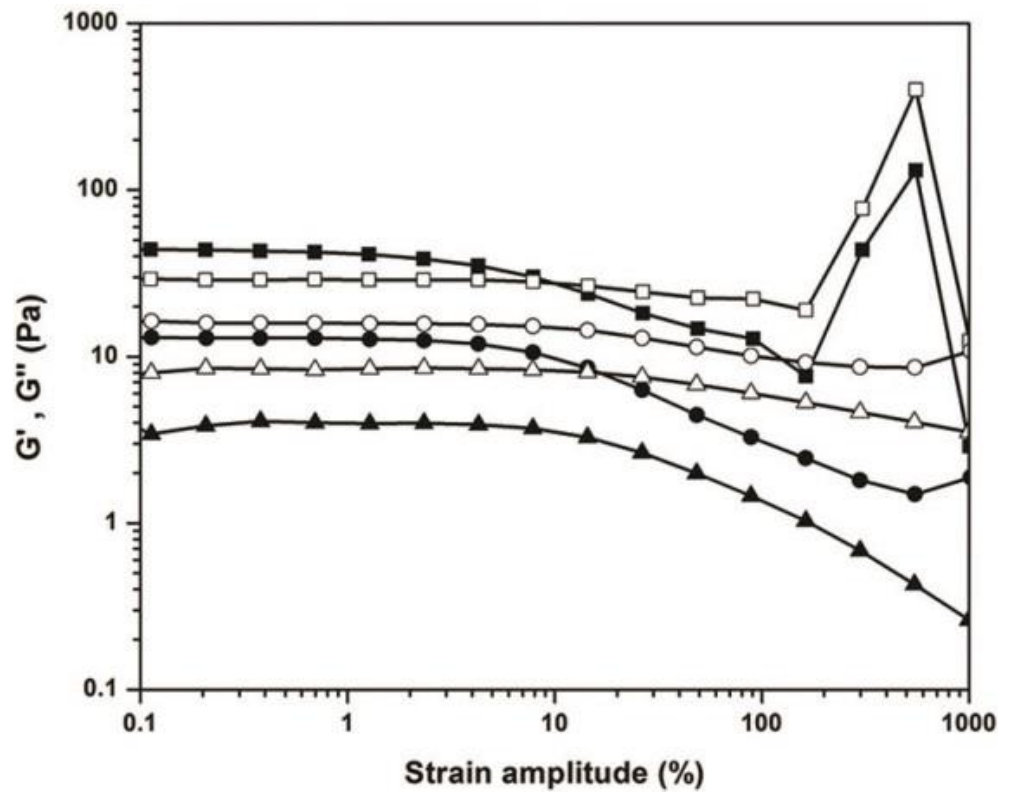

Figure 39. Storage and loss modulus as a function of strain amplitude (and $\omega=1 \mathrm{rad} / \mathrm{s}$ ) at different times of ultrasonication (at the last stage of fabrication process) and the same concentration of fumed silica particles in PEG (20\% w/w): $\mathbf{\square}, \square$, (STF-A), $45 \mathrm{~min} ; \boldsymbol{\bullet}, \bigcirc$, (STF-B), $45 \mathrm{~min} ; \boldsymbol{\Delta}, \triangle$, , (STF-C), 135 min; filled symbols: $\mathrm{G}^{\prime}$; empty symbols: $\mathrm{G}^{\prime \prime}$.

Figure 40 depicts the changes in oscillating dynamic moduli as a function of increasing imposed strain amplitude at a constant angular frequencies of $1 \mathrm{rad} / \mathrm{s}$ for STF-D, STF-E, and STF-F. For each of the samples, $\mathrm{G}^{\prime}$ exceeded $\mathrm{G}^{\prime \prime}$ at small strain amplitudes and a transition flow point was detected at 16,14, and 10\% strain amplitudes, respectively. The highest G' and G" values in the 
LVE domain was $100 \mathrm{~Pa}$ and $80 \mathrm{~Pa}$ in the case of STF-D, which demonstrates that the formed network-of-forces was weak and this sample represented a weak gel.

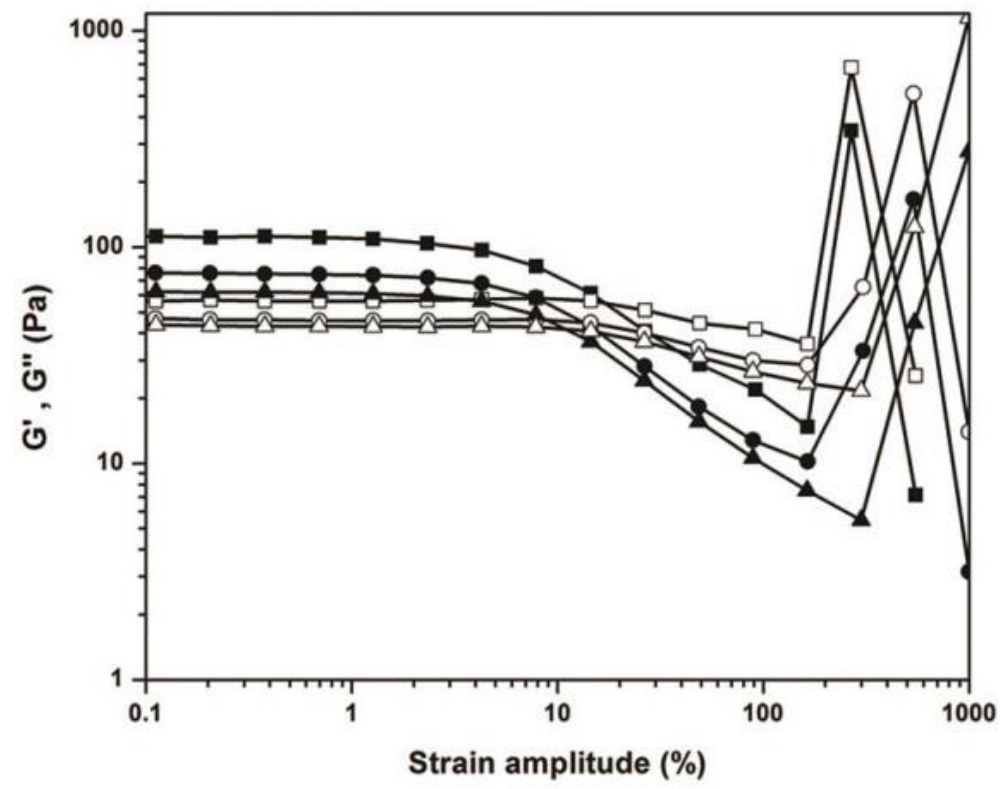

Figure 40. Storage and loss modulus as a function of strain amplitude (and $\omega=1 \mathrm{rad} / \mathrm{s}$ ) at different times of ultrasonication (at the last stage of fabrication process) and the same concentration of fumed silica particles in PEG (23\% w/w): $\boldsymbol{\square}, \square$, (STF-D), $45 \mathrm{~min}$; $\bullet$, $\bigcirc$, (STF-E), $45 \mathrm{~min} ; \boldsymbol{\Delta}, \triangle$, (STF-F), $135 \mathrm{~min}$; filled symbols: G'; empty symbols: $\mathrm{G}^{\prime \prime}$.

The colloidal microstructure of the STF samples in LVE region was analyzed in more detail at a constant strain amplitude of $1 \%$. Figure 41 depicts the changes in oscillating dynamic moduli as a function of increasing angular frequency for STF-A, STF-B, and STF-C. In the case of STF-A, $\mathrm{G}^{\prime}$ slightly exceeded $\mathrm{G}^{\prime \prime}$ through the whole range of angular frequencies while the maximum value of $\mathrm{G}^{\prime}$ exceeded up to $180 \mathrm{~Pa}$ at large motions. This behaviour showed that the sample behaved like a weak gel indicating that an unstable structured network of fumed silica particles was present at rest. In the case of STF-B and STF-C, G" exceeded G' at small angular frequencies, and a crossover point was detected at $\omega=3 \mathrm{rad} / \mathrm{s}$ and $\omega=35 \mathrm{rad} / \mathrm{s}$, respectively. This behaviour represented a non-flocculated viscous-like characteristic contributing to the stability of the dispersion at small angular frequencies. 


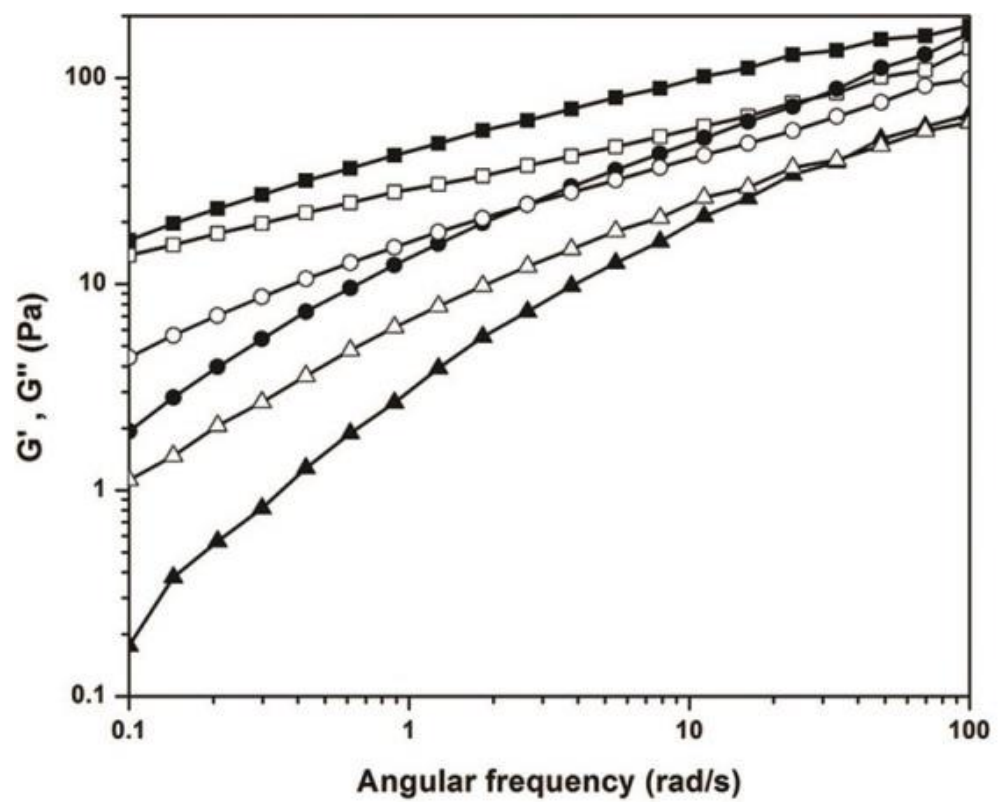

Figure 41. Storage and loss modulus as a function of angular frequency $(\gamma=1 \%)$ at different times of ultrasonication (at the last stage of fabrication process) and the same concentration of fumed silica particles in PEG (20\% w/w): $\mathbf{\square}, \square$, (STF-A), $45 \mathrm{~min} ; \boldsymbol{\bullet}, \mathrm{O}$, (STF-B), $45 \mathrm{~min} ; \boldsymbol{\Delta}, \triangle$, , (STF-C), 135 min; filled symbols: G'; empty symbols: G".

Figure 42 depicts the changes in oscillating dynamic moduli as a function of increasing angular frequency for STF-D, STF-E, and STF-F. In the case of STF-D and STF-E, G' slightly exceeded G" through the whole range of angular frequencies and the maximum value of G' exceeded up to $375 \mathrm{~Pa}$ and $270 \mathrm{~Pa}$ at large motions, respectively. In the case of STF-F, G' exceeded G" except at very small frequencies and a crossover point was detected at $\omega=0.15 \mathrm{rad} / \mathrm{s}$.

In general, the longer the last sequence of ultrasonication time or higher concentration of fumed silica particles in a given sample, a smaller the angular frequency for a gel-viscous transition and larger elastic modulus $\left(\mathrm{G}^{\prime}\right)$ at the highest angular frequency are observed. 


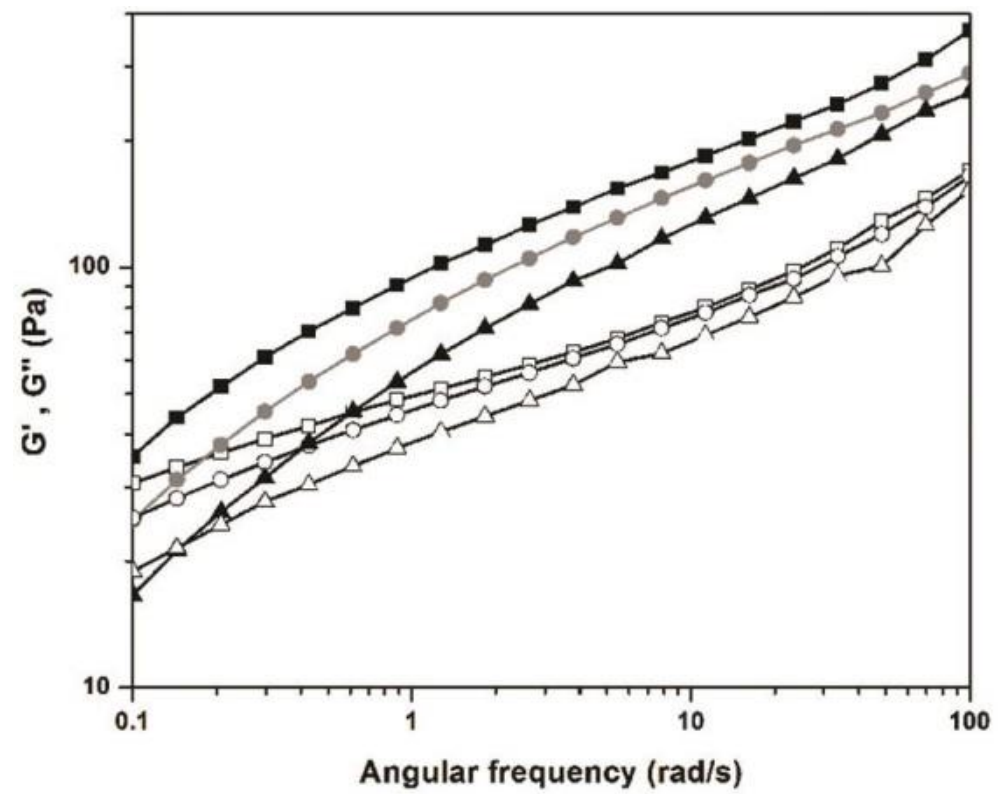

Figure 42. Storage and loss modulus as a function of angular frequency $(\gamma=1 \%)$ at different times of ultrasonication (at the last stage of fabrication process) and the same concentration of fumed silica particles in PEG (23\% w/w): $\mathbf{\square}, \square$, (STF-D) 23\%, $45 \mathrm{~min}$; $\bullet, O$, (STF-E) 23\%, $45 \mathrm{~min}$; $\boldsymbol{\Delta}, \triangle$, , (STF-F) 23\%, 135 min; filled symbols: G'; empty symbols: G'.

\subsubsection{Temperature-dependent Behaviour}

A temperature sweep test was conducted at a constant strain of $1 \%$ and a constant angular frequency of $1 \mathrm{rad} / \mathrm{s}$ for three continuous cycles to analyze the impact of temperature on the colloidal structure of the synthesized STF samples. For all the samples, both G' and G" moduli decreased with increasing temperature and increased by a subsequent cooling. It was observed that the particle concentration and the time period of ultrasonication could alter the impact of temperature on the colloidal system of the STF samples.

Figure 43depicts the changes in oscillating dynamic moduli as a function of time at different temperatures for STF-A, STF-B, and STF-C. In the case of STF-A and STF-B, G' slightly exceeded G" except at high temperatures. In the case of STF-C, G" exceeded G' through the whole range of temperature sweeps. 
Figure 44 depicts the changes in oscillating dynamic moduli as a function of time at different temperatures for STF-D, STF-E, and STF-F. In each of the samples, G' exceeded G" except at high temperatures. Under such a sequential temperature sweep, the temperatures at the crossover point of $G^{\prime}$ and $G^{\prime \prime}$ moduli were recorded for each sample, while $G^{\prime}$ was lower than $G^{\prime \prime}$ below a crossover point, and listed in Table 13.

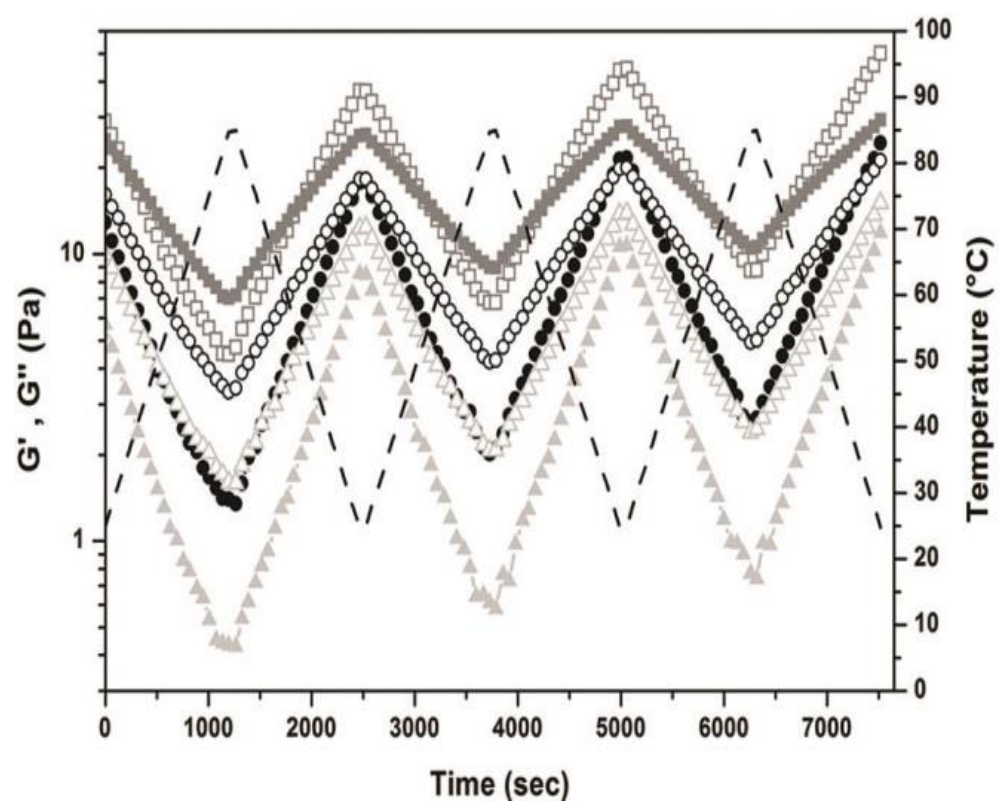

Figure 43. Storage and loss modulus as a function of temperature $(\gamma=1 \%, \omega=1 \mathrm{rad} / \mathrm{s})$ at different times of ultrasonication (at the last stage of fabrication process) and the same concentration of fumed silica particles in PEG (20\% w/w): $\mathbf{\square}, \square$, (STF-A), $45 \mathrm{~min} ; \boldsymbol{\bullet}, \bigcirc$, (STF-B), $45 \mathrm{~min}$; $\boldsymbol{\Delta}, \triangle$, (STF-C), 135 min; filled symbols: G'; empty symbols: G"; dash line: temperature. 


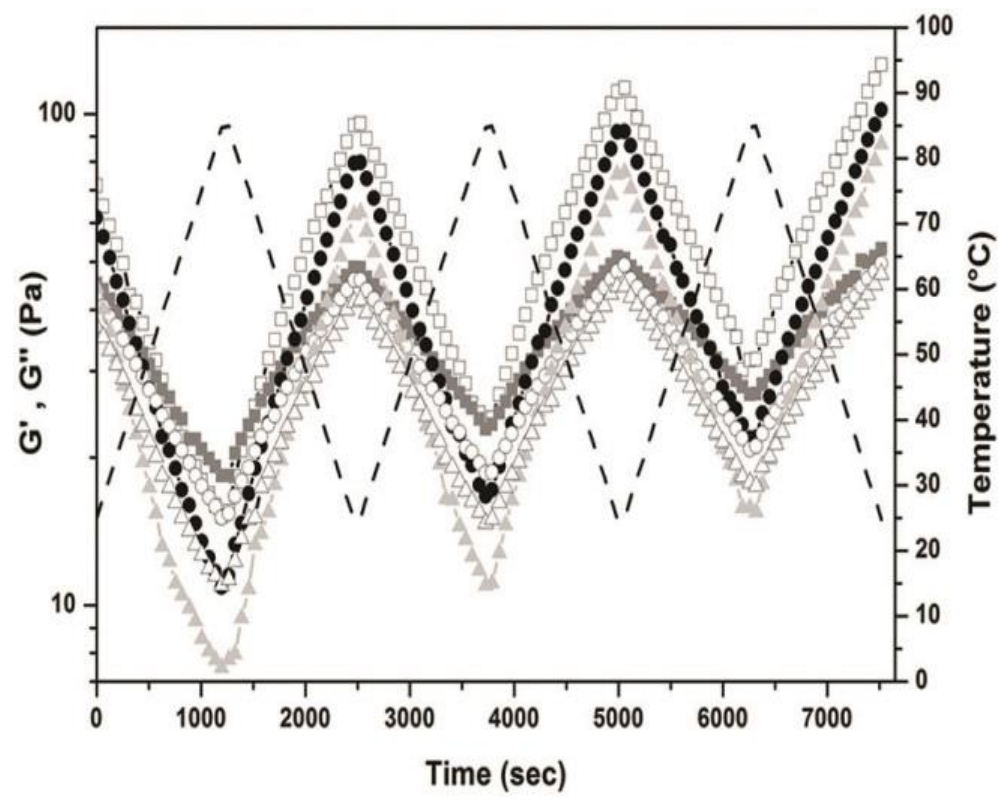

Figure 44. Storage and loss modulus as a function of temperature $(\gamma=1 \%, \omega=1 \mathrm{rad} / \mathrm{s})$ at different times of ultrasonication (at the last stage of fabrication process) and the same concentration of fumed silica particles in PEG (23\% w/w): $\boldsymbol{\square}, \square$, (STF-D), $45 \mathrm{~min} ; \boldsymbol{\bullet}, \mathrm{O}$, (STF-E), $45 \mathrm{~min} ; \boldsymbol{\Delta}, \triangle$, (STF-F), $135 \mathrm{~min}$; filled symbols: G'; empty symbols: G"; dash line: temperature.

Table 13. Temperatures at the crossover point of $G^{\prime}$ and $G^{\prime \prime}$ moduli for STF samples (temperature sweep oscillation at $\gamma=1 \%$ and $\omega=1 \mathrm{rad} / \mathrm{s}$ with the heating and cooling rate of $3{ }^{\circ} \mathrm{C} / \mathrm{min}$ for three cycles).

\begin{tabular}{|c|c|c|c|c|c|c|}
\hline \multirow[b]{3}{*}{ Sample } & \multicolumn{2}{|c|}{ First Cycle } & \multicolumn{2}{|c|}{ Second Cycle } & \multicolumn{2}{|c|}{ Third Cycle } \\
\hline & $25^{\circ} \mathrm{C}$ to $85^{\circ} \mathrm{C}$ & $85^{\circ} \mathrm{C}$ to $25^{\circ} \mathrm{C}$ & $25^{\circ} \mathrm{C}$ to $85^{\circ} \mathrm{C}$ & $85^{\circ} \mathrm{C}$ to $25^{\circ} \mathrm{C}$ & $25^{\circ} \mathrm{C}$ to $85^{\circ} \mathrm{C}$ & $85^{\circ} \mathrm{C}$ to $25^{\circ} \mathrm{C}$ \\
\hline & \multicolumn{6}{|c|}{ Temperatures $\left({ }^{\circ} \mathrm{C}\right)$ at the Crossover Point and $\mathrm{G}^{\prime}>\mathrm{G}^{\prime \prime}$ Below the Recorded Temperature } \\
\hline STF-A & 38 & 50 & 53 & 63 & 66 & 69 \\
\hline STF-B & \multicolumn{3}{|c|}{ No Crossover and $G^{\prime \prime}>G^{\prime}$} & 28 & 28 & 34 \\
\hline STF-C & \multicolumn{6}{|c|}{ No Crossover and $G^{\prime \prime}>G^{\prime}$} \\
\hline STF-D & 59 & 72 & & \multicolumn{3}{|c|}{ No Crossover and $G^{\prime \prime}>G^{\prime}$} \\
\hline STF-E & 53 & 69 & 69 & 78 & $G^{\prime}>G^{\prime \prime}$ & $\mathrm{G}^{\prime}>\mathrm{G}^{\prime \prime}$ \\
\hline STF-F & 38 & 56 & 56 & 66 & 69 & 75 \\
\hline
\end{tabular}

\subsubsection{Zeta potential measurement and SEM analysis}

Zeta potential measurements of the as received silica before and after ultrasonication were essentially the same. The zeta potential of fumed silica particles was $-35 \pm 1 \mathrm{mV}$ before and after ultrasonication. An SEM analysis of STF samples (Figure 45) shows changes in the morphology of fumed silica aggregates in the synthesized STF samples as a function of 
ultrasonication time and particle concentration: Figure 10(a) shows a branched-shape structure of agglomerates in as-received fume silica particles, Figure 45(b) shows the morphology of STF-D sample with $23 \%$ w/w of fumed silica particles in PEG, and Figure 45(c) shows the morphology of STF-A sample with $20 \% \mathrm{w} / \mathrm{w}$ of fumed silica particles in PEG. Overall, a more compact structure of fumed silica agglomerates was observed in STF-D compared with STF-A with the same time period of ultrasonication (at the latest stage). Additionally, Figures 45(c), 45(d), and 45(e) show the morphology of STF-B, STF-C, and STF-C* samples, respectively. All three samples contain $20 \% \mathrm{w} / \mathrm{w}$ of fumed silica particles in PEG and were exposed to different periods of ultrasonication (STF-B $<$ STF-C $<$ STF-C $*$ ) and compared.

In spite of observing the impact of ultrasonication time in reducing the size and compactness of agglomerates from a range of about $0.5 \mu \mathrm{m}$ in STF-A (20\% w/w and $45 \mathrm{~min}$ of ultrasonication) depicted in Figure 45(c) to the particle range size of about $0.2 \mu \mathrm{m}$ in STF-C (20\% w/w and 135 min of ultrasonication) depicted in Figure 45(e), it was not possible to estimate an accurate size distribution of fumed silica fractals from the SEM images due to the high degree of irregularities, nor it was possible at this time with particle size analyzer. 

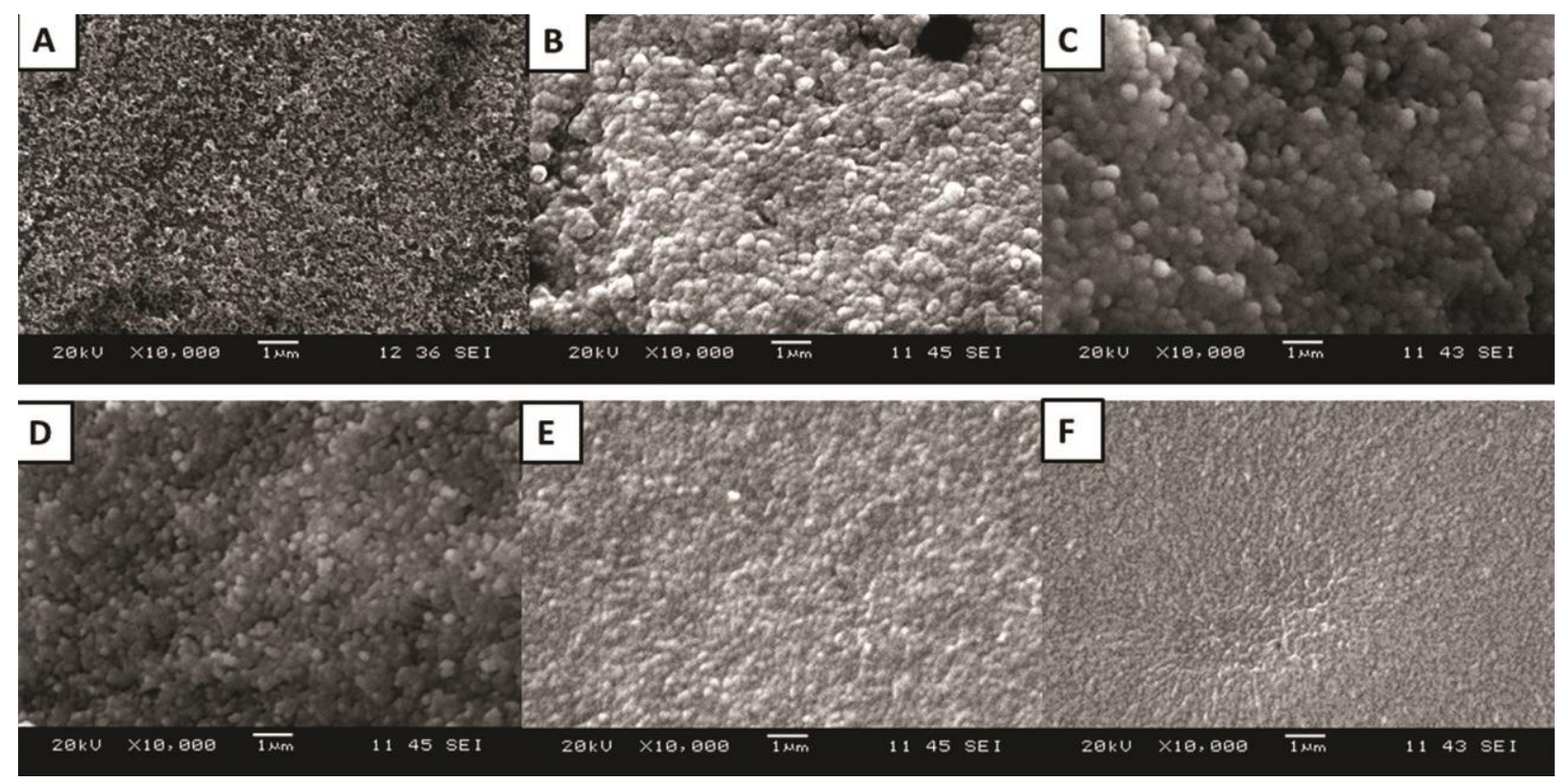

Figure 45. SEM images of fumed silica agglomerates after PEG medium was removed by heating from the dispersion, at different times of ultrasonication (at the latest stage of fabrication process) and different weight fractions of fumed silica particles: a) as-received fumed silica; b) (STF-D), 23\% w/w, $45 \mathrm{~min}$; c) (STF-A), 20\% w/w, $45 \mathrm{~min}$; d) (STF-B), 20\% w/w, $90 \mathrm{~min}$; e) (STF-C), 20\% w/w, $135 \mathrm{~min}$; f) (STF-C*), $20 \% \mathrm{w} / \mathrm{w}, 450 \mathrm{~min}$.

\subsubsection{Discussion on the Rheological Properties of Fumed Silica Particles}

Since the structure of fumed silica aggregates is irregular and anisotropic, the clustering theory is more likely acceptable to explain the shear thickening behaviour of fumed silica dispersions rather than the ODT mechanism $[73,83,84,103]$. Fumed silica can contribute to the greatest extent to the flow behavior and high viscosity of colloidal dispersions because of the unique elongated shape of aggregates and the presence of considerably high hydrodynamic interparticle forces when compared to spherical particles [73,104]. The occurrence of shear thickening phenomenon in fumed silica dispersions is more likely attributed to the transient shear-induced bridging of particle aggregates and a three-dimensional network structure which spans throughout the system $[101,104,126]$. From the results, it was observed that the sample with a lower concentration of fumed silica particles or longer time period of ultrasonication exhibited a lower $\eta_{\max }$ but a higher $\dot{\gamma}_{c}$ (see Section 4.2.1). This kind of behaviour was likely attributed to the 
weakened hydrodynamic interparticle interactions and the formation of smaller hydroclusters at the onset of shear thickening. The sample with a lower $\eta_{\max }$ but a higher $\dot{\gamma}_{c}$ also showed the G" domination over $\mathrm{G}^{\prime}$ across a greater domain of angular frequencies or strain amplitudes and possessed a lower flow stress showing a viscous response under dynamic shear. From the literature, this rheological behaviour (i.e., $G^{\prime \prime}>G^{\prime}$ ) is indicative ofthe stability and non-flocculated nature of dispersions $[73,84]$. Additionally, the sample with a higher concentration of fumed silica particles or shorter time period of final ultrasonication showed G' domination over G" across a greater domain of angular frequencies or strain amplitudes and possessed a higher flow stress. From the literature, this rheological behaviour (i.e. $\left.G^{\prime}>G^{\prime \prime}\right)$ signifies that a threedimensional network structure is present in the system and highlights its colloidal gel-like characteristic $[73,84,106]$. From zeta potential measurements, it was found that an intensive ultrasonication did not cause a change in the potential charges of fumed silica particles (see Section 4.2.5). From the literature, a key factor to control the gelation/stability of fumed silica dispersions in a variety of different liquids is the degree of hydrogen bonding between silica particles and also the electrostatic repulsion of the silica surface [103]. Therefore, the impact of ultrasonication to alter the viscoelastic characteristics of STF samples was most likely attributed to the weakening of hydrogen bonding but not the changes of electrostatic repulsion forces. From the SEM images in Section 4.2.5, it was observed that increasing the time period of ultrasonication helped disrupt agglomerates into smaller clusters. The sample with higher particle concentration (i.e. $23 \%$ versus $20 \%$ ) and the same time period of ultrasonication of the final sequence of fabrication process also showed a denser structure. From the literature, the dispersion state of fumed silica is directly dependent on the degree of interparticle interactions. Hence, a well-dispersed system behaves less viscous-like, with weaker particle-particle 
interactions, higher shear modulus at low deformation, and a shorter linear viscoelastic domain [109]. Therefore, the loose microstructural morphology of an STF sample which exhibited a prominent viscous-like behaviour was correlated to lower interparticle interactions. Hydrogen bonding is the key factor controlling the viscoelastic behavior and stability of fumed silica dispersions in a variety of different solvents [103].

In general, two extreme classes of fumed silica dispersions were identified: (i) A low viscous and stable system where strong hydrogen bonding is present between the hydroxyl groups on particle surface and liquid medium and (ii) An elastic colloidal gel where strong hydrogen bonding is absent with the dispersing medium while adjacent nano size chain aggregates (NCA) interact with each other directly through hydrogen bonding and form particle flocculation $[73,84,106,107]$. However, if the dispersing medium is polar (such as the PEG used in this research), hydrogen bonding occurs more preferably between the molecules of liquid medium and surface hydroxyls present on fumed silica particles [26,73]. Nevertheless, even after dispersing fumed silica particles in a polar medium, some aggregation of fumed silica particles could occur [26]. Hydrogen bonding between fumed silica particles arise with a higher concentration of particles, which can result in the formation of a structured network [105]. Hence, a limit could be defined for the maximum volume fraction of hard particles in various colloidal dispersions which allows the shear thickening phenomenon to exhibit. This restriction is related to the tendency of nanoparticles (e.g. fumed silica particles) to agglomerate $[127,128]$. In other words, the stability of dispersions is governed by the strength of particle-particle and particle-liquid interactions [101]. As the colloidal volume fraction increases, interparticle interactions dominate and control the rheological properties and stability of the dispersion [129]. Therefore, it was postulated that the applied ultrasonic treatment in this research could change 
the rheological characteristics of STF samples by reducing the hydrogen bonding between silica particles, resulting in the samples becoming more viscous-like after ultrasonication. Temperature control can significantly influence the rheological properties of fumed silica colloidal dispersions $[101,107,108,130]$. From previous studies, the temperature-dependent behaviour of samples was a usual phenomenon: showing more viscous-like characteristics at high temperatures (see Section 4.2.4). From the literature, this kind of behaviour was attributed to a lower degree of hydrogen bonding and colloidal interactions at high temperatures [101,107].

From the results, after the cooling cycle, G' increased to a higher level than its initial state. Retesting the sample for the second and the third sweeps of heating and cooling cycles resulted in a continuous increase of $\mathrm{G}^{\prime}$, at the end of each cooling cycle, when it was compared to its initial state. Additionally, G' and G" crossed over at a different temperature in each temperaturesweep cycle. A plausible explanation for this behaviour is that the sample became less viscouslike after each heating and cooling cycle while a metastable gel-like structure was induced in the system. It was also observed that samples fabricated with a longer final period of ultrasonication exhibited a lower increase of the elastic modulus $\left(G^{\prime}\right)$ after heating and cooling cycles and showed a lower viscous-to-gel transition temperature. This presumably is due to the weaker hydrogen bonding between silica particles. It was also demonstrated that the rheological measurements for samples reported in Section 4.2 were reproducible in a three-month period. This demonstrates that the effect of ultrasonication on the rheological properties of samples was long-lasting.

\subsubsection{Discussions \& Summary}

A hydrophilic type of fumed silica was used to fabricate colloidal dispersions in PEG using sequential ultrasonic treatment. A set of steady-state and dynamic rheological measurements 
were performed on STF samples to investigate the effect of ultrasonication time on the rheological properties of samples. From the rheological results, it was demonstrated that the time period of ultrasonication in the fabrication process of STF materials was influential regarding viscoelastic characteristics. From the SEM analysis, it was shown that the network of particle clusters disrupted by ultrasonication into smaller agglomerates. Hence, this treatment could help disperse a large content of fumed silica particles in PEG, presumably by reducing interparticle interactions. As a consequence, a weaker interaction between fractal fumed silica clusters turned the dispersion into a pronounced viscous-like state with less gel-like behavior correlated to the prominent stability of the dispersion.

It was also found that the impact of ultrasonication was not due to the change of electrostatic interactions between particles as zeta potential did not change in this treatment. Additionally, the critical shear rate at the onset of shear thickening was increased by increasing the ultrasonication time while the maximum shear thickened viscosity was retarded. The time periods of ultrasonication induced a significant impact to the temperature-dependent behaviour of STF samples. It was observed that the elastic modulus $\left(\mathrm{G}^{\prime}\right)$, after the cooling cycle, returned closer to its initial state in samples which exposed longer to ultrasonication, while its viscous-to-gel transition point happened at a lower temperature. Since the results were reproducible in a threemonth period, it was concluded that such an intensive ultrasonication could tailor the rheological characteristics and stability of fumed silica dispersions with long-lasting effects.

\section{The Effectiveness of Nylon Coating on UHMWPE Fabric to Host STF Materials}

At this time, no successful approach that incorporated UHMWPE fabric with STF materials is demonstrated. There are several methods already reported that may improve the wettability of UHMWPE polymer to allow bonding with other materials in the manufacturing of composite 
materials (e.g. chemical etching or plasma irradiation) especially for clinical applications. On the other hand, there are some drawbacks of these treatments like the loss of toughness or tensile strength (see Section 5.1). As was mentioned in Section 4.1.1, the mechanical interlocking of nylon could create a uniform and stable coating on the inherently inert UHMWPE fibre/fabric as a result of nylon film shrinkage upon removal of $\mathrm{CaCl}_{2}$ and drying. Efforts were then made to develop a new material based on UHMWPE fibre combined with STF materials, and to confirm if STF materials could be compatible with the nylon coated UHMWPE.

The synthesized STF material was impregnated into the fabric samples by two different techniques: (i) Diluting the STF material with ethanol alcohol to facilitate its impregnation into fabric (by reducing surface tension). Till now, this technique is the only successful way to facilitate impregnating STF materials with fabric and (ii) Heating STF materials at $80-100{ }^{\circ} \mathrm{C}$ for a short period of time (about $5 \mathrm{~min}$ ) to facilitate impregnating STF into fabric samples. As was discussed in Section 4.2.4, the STF samples showed more viscous-like characteristics at high temperatures. The diluted STF material was then poured into a polyethylene bag with the UHMWPE fabric inside. A manual rubber coated nip roller was used to squeeze out excess STF material and to improve the impregnation process, shortly after the addition of STF while it was still hot and dilute. The STF-fabric sample was then removed from the oven and kept inside the sealed bag before the impact test.

Prior to the application of either of these two techniques, it was confirmed that both alcohol dilution or heating samples had a negligible effect on the rheological properties of STF materials before and after the treatment. In this research, fumed silica particles were used to fabricate STF material, in comparison to previous studies which have used monodisperse colloidal silica 
particles. The rheological properties of synthesized STF were studied in details (see Section 4.2.1 to Section 4.2.4).

The SEM image of neat UHMWPE fabric and nylon coated UHMWPE fabric samples impregnated with STF material is depicted in Figure 46(a) and 46(b), respectively. From the SEM images, it was observed that the adhesion of STF material to the nylon coated UHMWPE fabric sample is more dense and noticeable in the case of nylon coated UHMWPE fabric sample. Despite the visual effectiveness of integrating STF material with fabrics, as the fabric turns to rigid state in a fast motion, this treatment did not increase the penetration resistance of neat UHMWPE fabric sample on the same areal density basis (from the dynamic impact test). Similarly, the addition of STF did not add penetration resistance to nylon coated UHMWPE fabric sample. Increasing the loading of fumed silica particles to $32 \% \mathrm{w} / \mathrm{w}$ of the dispersion was not helpful either. It was then interpreted that the chain-like structure of fumed silica particles is the most likely reason which makes such an STF material undesirable for this application.
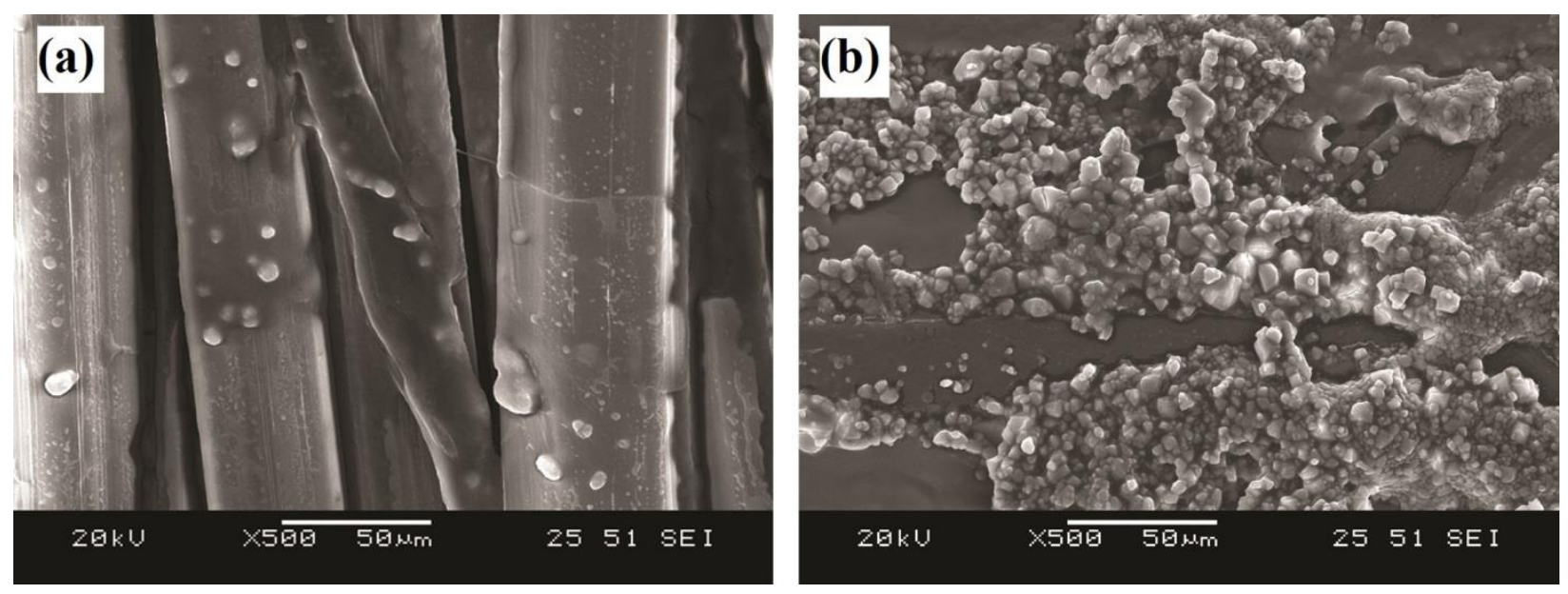

Figure 46. SEM images: (a) STF impregnated neat UHMWPE fabric, (b) STF impregnated Nylon 6,6 coated UHMWPE fabric (the STF material is comprised of $23 \% \mathrm{w} / \mathrm{w}$ amorphous fumed silica dispersion in PEG-200). 


\section{CHAPTER 5 BIOLOGICAL APPROACH; A POTENTIAL APPLICATION}

In a continuing effort to evaluate the mechanical and physical properties of the materials developed in this research, the potential biomedical application for nylon coated UHMWPE was investigated. This chapter presents the biological assessment of the materials developed in Section 3.1. As a result, this chapter provides additional information concerning the biocompatibility of the nylon coated UHMWPE that may be useful for the manufacturing of clinical devices. First, a concise review will be presented concerning the biological aspects of UHMWPE, including recent literature reviews of the two main issues with the load-bearing prosthesis made of UHMWPE composite materials; (1) High creep susceptibility of this polymer, which was also addressed in Section 3.1.7 and (2) The generation of wear debris and the induction of cytotoxicity. In this chapter, the toxicity of the neat and nylon coated UHMWPE was verified and compared by in-vitro cytotoxicity assessment, followed by an investigation of the induced-osteolysis effect (as an indicator of wear debris generation), followed by an apoptosis assay. Finally, the experimental results and the corresponding explanations of the biological testing will be presented and a detailed discussion of the results will follow.

\subsection{Introduction; Biological Aspects of UHMWPE Material}

UHMWPE is being extensively used as a successful load bearing material in orthopedic applications due in part to its superior properties of biocompatibility, lightweight, good wear resistance, chemical stability, and lubricity which fit the biological and mechanical requirements. However, UHMWPE's properties strongly depend on its synthesis and processing conditions which may alter the overall performance of the implanted device [43]. Despite the relative high abrasion resistance of UHMWPE, wear debris and the induction of cytotoxicity due to leachable eluates from the material can limit the long term performance of total hip and knee replacement 
prostheses. The most common reasons for an implant failure is osteolysis (i.e. a painful inflammatory reaction) due to wear debris and stress shielding induced by a stiff material [131,132]. In general, it would be beneficial to provide a body implant with higher strength and lower stiffness [133].

In general, the implants should have high strength and low stiffness to allow the underlying bone to carry a considerable amount of load, while having high wear resistance to prevent osteolysis at joints (i.e. an articulating component) and bone-implant interface [133]. Several studies have evaluated the wear resistance of UHMWPE polymer and identified it as the primary cause of osteolysis and subsequent aseptic loosening of implants. In order to minimize the wear of UHMWPE, several solutions were introduced, such as irradiation cross-linking, and composite technology. On the other hand, little improvement in the wear resistance of UHMWPE was observed when it was reinforced with fillers (e.g. glass or aramid fibres), however, substantial improvement was achieved by extensive gamma radiation cross-linking and thermal processing [43].

The longitudinal tensile properties and creep resistance of GUR-405 UHMWPE can be improved by reinforcement with UHMWPE fibre (e.g. Spectra-1000), although, the wear resistance was not improved and the transverse mechanical properties were even degraded significantly [134,135]. Similarly, the wear resistance of UHMWPE GUR-410HP became lowered or unchanged after the reinforcement with UHMWPE fabric because of poor fibre-matrix adhesion [136]. The wear resistance of HDPE/UHMWPE composite could be improved after reinforcement with multi-walled carbon nanotubes (MWCNTs) [137]. Nevertheless, Because of inconsistency in results and difficulties in the manufacturing process of UHMWPE 
homocomposite, this type of composite has not been commercialized for load-bearing orthopedic applications [43].

The wear resistance of UHMWPE filled with micron-sized quartz particles could also be improved using an organosiloxane coupling agent which increased the adhesion between the particles and the matrix [138]. A treatment with gamma irradiation can decrease the wear rate of UHMWPE. It was also proved that the recrystallization of UHMWPE could be helpful to enhance polymer cross-linking after gamma irradiation. In results, the tensile strength of the irradiated recrystallized sample was reduced by increasing the radiation dose. On the other hand, the elongation of UHMWPE was lowered by increasing the radiation dose because of enhanced cross-linking of the polymer chains [139]. On the contrary, it was reported that gamma irradiation can enhance the tensile and compressive creep performance of GUR-1050 UHMWPE [140].

In general, the long term stability of body implant depends on the creep characteristics and wear resistance of the component which maintain its dimensions. UHMWPE, which is a viscoelastic material, is inherently weaker in creep and fatigue resistance than metal stem and cortical bone [43]. It was shown that the creep rate of UHMWPE implants under the continuous stress (i.e. body weight) is a critical factor in orthopedic applications [141]. The creep rate of polyethylene acetabular liner has an important effect on early penetration and wear rate due to the smaller head-liner contact area after total hip arthroplasty (THA); at the same time it was found to have much less influence on the critical factor of long term volumetric wear rate [142]. The creep rate has a significant influence on the penetration rate of the polyethylene femoral condyle implant. It was found that the creep rate could be dramatically increased if the implanted device is thin or misaligned, or if the patient is overweight [143]. 
The poor adhesion of UHMWPE is cited as the primary reason of inadequate consolidation and short term clinical failure in carbon fibre reinforced UHMWPE composites [43]. Various methods have been tried to resolve this type of failure. For instance, the mechanical and tribological properties of aramid fibre reinforced UHMWPE composite could be improved by plasma irradiation or chemical etching by improving fibre-matrix interfaces [46].

The cross-linking of UHMWPE by gamma irradiation could enhance the wettability and wear resistance of UHMWPE, although, this improvement is associated with some important setbacks, such as the degradation of other mechanical properties (e.g. toughness) and increasing the vulnerability of the UHMWPE to oxidation due to the generation of free radicals [43]. As another example, it was demonstrated that the surface modification of UHMWPE fibre by plasma and chemical treatments could improve the tensile properties of the fibre, although, its elongation reduced significantly [144]. On the contrary, it was reported that the mechanical properties of UHMWPE fibre remained unchanged after gamma irradiation or chemical treatment, while the fibre could become functionalized and able to bond with other matrix materials [145].

The negative effects of gamma irradiation could be compensated by adding carbon nanotubes (CNTs) to the UHMWPE matrix $[146,147]$. The problem of using CNT is that this substance is cytotoxic with the characteristic features of cell necrosis and degeneration [148], while CNT was considered as a biocompatible material in another study [149]. A bioactive sprayed hydroxyapatite (HA) coating over alumina ceramic particulate reinforced UHMWPE composite could facilitate biological fixation between the prosthesis and the hard tissue. The integrity and long term stability of such a composite could also be improved by this coating [150]. A titanium 
or titanium/HA coating is another option on UHMWPE which can stabilize bearing surfaces of the arthroplasty device and improve the osteoblastic interface with bone [151].

\subsection{Biological Tests}

\subsubsection{Preparation of Neat and Nylon 6,6 Coated UHMWPE Extracts}

An extract was prepared from either the neat and Nylon 6,6 coated UHMWPE fibre according to the ISO standards (10993-5: 1999) related to in vitro cytotoxicity test method in a similar research study [152]: The fibre sample was sliced into $2.5 \mathrm{~cm}$ length pieces and sterilized in $97 \%$ ethanol for $5 \mathrm{~min}$, then washed twice with phosphate buffered saline (PBS), and finally left to air dry for $15 \mathrm{~min}$. This sterilized material was then immersed in Dulbecco's Modified Eagle Medium (DMEM) at a ratio of $2.5 \mathrm{~cm} / 3 \mathrm{~mL}$ and incubated under $5 \% \mathrm{CO}_{2}$ in the air for $72 \mathrm{~h}$ at 37 ${ }^{\circ} \mathrm{C}$ using an orbital shaker at constant agitation of 200 RPM. The medium was harvested by filtration then kept at $-80{ }^{\circ} \mathrm{C}$ until used.

The cytotoxicity test was not performed on the Nylon 6,12 coated UHMWPE fibre due to material limitations. Moreover, Nylon 6,6 and Nylon 6,12 have similar chemical composition as it was discussed in section 3.1.2.

\subsubsection{Cell Culture for L929 Fibroblasts and U-937 Human Macrophage Cell Lines}

The L929 fibroblast cell line was used in this study to investigate the cytotoxicity of the samples as this type of cell allows for the study of certain common cellular functions, such as membrane state, viability, proliferation, attachment, and cellular properties. These cellular functions are related to the basal cytocompatibility of biomaterials. In addition, the L929 fibroblast cell line is in the direct contact with orthopedic implants during clinical use [153]. Similar to osteoblasts, osteoclasts, and macrophages, fibroblasts are among the major cells that populate the bone implant interface and play an important role in bone osteolytic activity [154-156]. 
The L929 cell line (ATCC) was grown in DMEM (Lonza, USA) which was supplemented with $10 \%$ fetal bovine serum (FBS; Lonza, USA) and 5\% penicillin/streptomycin (Lonza, USA). The cells were seeded at $3 \times 10^{4}$ cells/well in a 96-well plate and left under $5 \% \mathrm{CO}_{2}$ in the air for $24 \mathrm{~h}$ at $37{ }^{\circ} \mathrm{C}$ in order to adhere and attach. The culture medium was then replaced by the prepared "conditioned media" for both the neat and Nylon 6,6 coated UHMWPE after supplementing it with 10\% FBS and 5\% penicillin/streptomycin. Two controls were used: (i) The L929 cells treated with fresh complete media, which has not been in contact with the test material yet, and (ii) The L929 cells treated with a shaken media, which has not been in contact with the test material yet, and processed under the same conditions applied in the preparation of the conditioned media. The reason to prepare the second control was to test if the agitation process alone affected cell survival or not. The treated and untreated cells were incubated under a humidified atmosphere of $5 \% \mathrm{CO}_{2}$ in the air for 24,48 , and $72 \mathrm{~h}$ at $37{ }^{\circ} \mathrm{C}$.

The U-937 human macrophages were used to investigate the osteolysis induced by wear debris. These cells were cultured in Roswell Park Memorial Institute (RPMI) 1640 Medium supplement with $10 \%$ FBS and 5\% penicillin/streptomycin in a standard cell culture environment which is $5 \% \mathrm{CO}_{2}$ in the air at $37{ }^{\circ} \mathrm{C}$. The cells were incubated for $24 \mathrm{~h}$ in T-25 flasks with $10 \mathrm{~mL}$ conditioned media also formulated by the addition of $10 \% \mathrm{FBS}$, and $10 \%$ penicillin/streptomycin at the cell density of 100,000 cells/mL media/well. The cells were harvested from $2 \mathrm{~mL}$ of cell suspension culture after 24, 48, and $72 \mathrm{~h}$ for RNA extraction. RNA extraction was performed using Trizol (Invitrogen). The qualification of RNA extract was then confirmed at $260 \mathrm{~nm}$ on Shimadzu Spectrophotometer UV-1800.

The L929 mouse fibroblasts were used to investigate the apoptosis induced by the neat and nylon coated UHMWPE samples. L929 mouse fibroblasts were cultured in DMEM, supplemented with 
$10 \% \mathrm{FBS}$, and $5 \%$ penicillin/streptomycin in a standard culture condition, which is $5 \% \mathrm{CO}_{2}$ in the air at $37{ }^{\circ} \mathrm{C}$. The cells were incubated with the conditioned medium for $24 \mathrm{~h}$ at the cell density of 50,000 cells $/ 0.5 \mathrm{ml}$ media/well. The cells were then harvested from $2 \mathrm{~mL}$ of cell suspension culture after 24,48 , and $72 \mathrm{~h}$ for RNA extraction. RNA extraction was performed using Trizol (Invitrogen). The qualification of RNA extract was then confirmed at $260 \mathrm{~nm}$ on Shimadzu Spectrophotometer UV-1800.

\subsubsection{Cytotoxicity Assay by MTT}

The test materials were assessed in vitro on L929 cells using the Methyl thiazol Tetrazolium (MTT) assay for the cytotoxicity test [157]. The MTT assay is based on the ability of living cells

to convert a water-soluble yellow dye, 3-(4,5-dimethylthiazole-2-yl)-2,5-diphenyl tetrazoliumbromide, into violet formazan crystals by the action of mitochondrial dehydrogenase enzymes which presents in living cells, while the intensity of violet color produced is proportional to cell number.

The conditioned media of cells were discarded after 24, 48, and $72 \mathrm{~h}$, and replenished with 120 $\mu \mathrm{L}$ MTT solution composed of $100 \mu \mathrm{L}$ complete media plus $20 \mu \mathrm{L}$ MTT $(5 \mathrm{mg} / \mathrm{mL})$. The cells were incubated in the MTT solution under a humidified atmosphere of $5 \% \mathrm{CO}_{2}$ for $4 \mathrm{~h}$ at $37{ }^{\circ} \mathrm{C}$. The MTT solution was then removed and the formazan crystals were solubilized with $100 \mu \mathrm{L}$ DMSO and the absorbance was measured at $544 \mathrm{~nm}$ using a microplate reader FLUOstar OPTIMA (BMG LabTech, Germany). The cytotoxicity of the sample was calculated as the percentage of the control cell viability while the control cells were considered to be of $100 \%$ survival. 


\subsubsection{Investigation of Osteolysis by RT-PCR}

$1 \mu \mathrm{g}$ of the extracted RNA was used as a template for synthesizing cDNA using RevertAid ${ }^{\mathrm{TM}}$ First Strand cDNA Synthesis Kit (Fermentas, Thermo Fisher Scientific) in accordance to the manufacturer protocol. The polymerase chain reaction (PCR) was then performed to amplify tumor necrosis factor alpha (TNF $\alpha$ ) and interleukin 6 (IL-6) genes as osteolysis markers from the U-937 human macrophages and the housekeeping $\beta$-actin as the endogenous loading control. The PCR program comprised of an initial denaturation for 3 min at $94{ }^{\circ} \mathrm{C}$, followed by 35 cycles of $30 \mathrm{~s}$ denaturation at $94{ }^{\circ} \mathrm{C}$, annealing for $30 \mathrm{~s}$ at $72{ }^{\circ} \mathrm{C}$ and extension for $45 \mathrm{~s}$, and a final extension for $10 \mathrm{~min}$. The PCR product was then analyzed by Agarose gel electrophoresis.

\subsubsection{Investigation of Apoptosis by RT-PCR}

$1 \mu \mathrm{g}$ of the extracted RNA was used as a template for synthesizing cDNA using RevertAid ${ }^{\mathrm{TM}}$ First Strand cDNA Synthesis Kit (Fermentas, Thermo Fisher Scientific) in accordance to the manufacturer protocol. The PCR was then performed to amplify Caspase3 and Bax genes as apoptosis markers from the L929 mouse fibroblasts and the house keeping $\beta$-actin as the endogenous loading control. The PCR program comprised of an initial denaturation for $3 \mathrm{~min}$ at $94{ }^{\circ} \mathrm{C}$, followed by 35 cycles of $30 \mathrm{~s}$ denaturation at $94{ }^{\circ} \mathrm{C}$, annealing for $30 \mathrm{~s}$ at $72{ }^{\circ} \mathrm{C}$ and extension for $45 \mathrm{~s}$, and a final extension for $10 \mathrm{~min}$. The PCR product was then analyzed by Agarose gel electrophoresis.

\subsubsection{Statistical Analysis and Statistical Comparison}

The results of PCR gel electrophoresis were analyzed and normalized to the internal control $\beta$ actin using ImageJ-Software (National Institute of Health, USA). The expression values of the tested genes were calculated in cells cultured in conditioned media with the neat and nylon coated UHMWPE material relative to their expression in the media conditioned without any 
material. The results were compared using one-way ANOVA (Analysis of Variance), followed by Tukey-Kramer Multiple Comparisons Test using GraphPad Prism-5 software. Raw data, corrected with respect to the blank reading, was used as the input value for the statistical analysis program $\mathrm{R}^{\odot}$ version 2.14.1 (2012-12-22) for the MTT assay. P-values for significance were computed using ANOVA (Analysis of Variance) and p-values $<0.05$ were considered significant.

\subsection{Results and Discussions}

\subsubsection{MTT Results}

From Figure 47, MTT assay after exposing L929 fibroblast cells in the conditioned medium of neat and Nylon 6,6 coated UHMWPE material was compared with two controls for three incubation periods $(24,48$, and $72 \mathrm{~h})$, as the mean of cell survival percentage in each condition. The cells in contact with the fresh unconditioned medium were considered as the control of $100 \%$ viability. At $24 \mathrm{~h}$ incubation period, from the MTT assay, it was demonstrated that the cell survival after exposure to the shaken medium, neat UHMWPE medium, and Nylon 6,6 coated UHMWPE medium was 93.9, 73.4, and 83\%, respectively. At $48 \mathrm{~h}$ incubation period, the cell survival was $87.5,54.8$, and $72.4 \%$ in the case that cells exposed to shaken medium, neat UHMWPE medium, and Nylon 6,6 coated UHMWPE medium, respectively. These results come in concordance with those of the $24 \mathrm{~h}$ incubation period. A similar pattern in the percentage of cell survival was detected at $72 \mathrm{~h}$ incubation period: the cell survival was $85.9,53.3$, and $80.7 \%$ in the case that cells exposed to shaken medium, neat UHMWPE medium, and Nylon 6,6 coated UHMWPE medium, respectively. In general, it was demonstrated that cells treated with Nylon 6,6 coated UHMWPE medium showed higher viability compared to cells treated with neat UHMWPE medium. 
It was also indicated that the decrease in survival of the cells treated with neat UHMWPE was significant when compared to: (i) The control for three incubation periods ( $\mathrm{p}<0.001)$ and (ii) The cells treated with the shaken medium in the case of $24 \mathrm{~h}$ incubation period $(\mathrm{p}<0.01)$, while $\mathrm{p}<0.001$ in the case of $48 \mathrm{~h}$ and $72 \mathrm{~h}$ incubation periods. Also, the decrease in survival of the cells treated with Nylon 6,6 coated UHMWPE medium was significant when compared to: (i) The control for $24 \mathrm{~h}$ incubation period $(\mathrm{p}<0.01)$, while $\mathrm{p}<0.001$ in the case of $48 \mathrm{~h}$ incubation period, and $\mathrm{p}<0.05$ in the case of $72 \mathrm{~h}$ incubation period and (ii) The cells treated with the shaken medium for $48 \mathrm{~h}$ incubation period only $(\mathrm{p}<0.01)$.

In the results, it was demonstrated that neat UHMWPE material induced significant cell death compared to the control with the same $\mathrm{p}$-value $(\mathrm{p}<0.001)$ for three incubation periods. Also, Nylon 6,6 coated UHMWPE material induced significant cell death compared to the control with different p-values for three incubation periods. Overall, it was indicated that neat UHMWPE material caused a more significant decrease in cell survival than Nylon 6,6 coated UHMWPE material in relation to the control.

Moreover, there was no significant difference in survival of cells treated with the shaken medium without the material and the control except at $48 \mathrm{~h}$ incubation period $(\mathrm{p}<0.01)$, which indicates that the process of conditioning the medium through shaking had no significant effect on the cell death. In other words, any decrease of the cell survival was due to the material tested and not the shaking process itself.

Finally, the decrease in survival of cells treated with neat UHMWPE medium was significant to that of the cells treated with Nylon 6,6 coated UHMWPE medium for $48 \mathrm{~h}$ incubation period ( $\mathrm{p}$ $<0.001)$ and $72 \mathrm{~h}$ incubation period $(\mathrm{p}<0.01)$. This shows that neat UHMWPE material induced more significant cell death compared to Nylon 6,6 coated UHMWPE material. Accordingly, 
Nylon 6,6 coated UHMWPE material is less cytotoxic, which means that nylon could improve the biocompatibility of UHMWPE material.

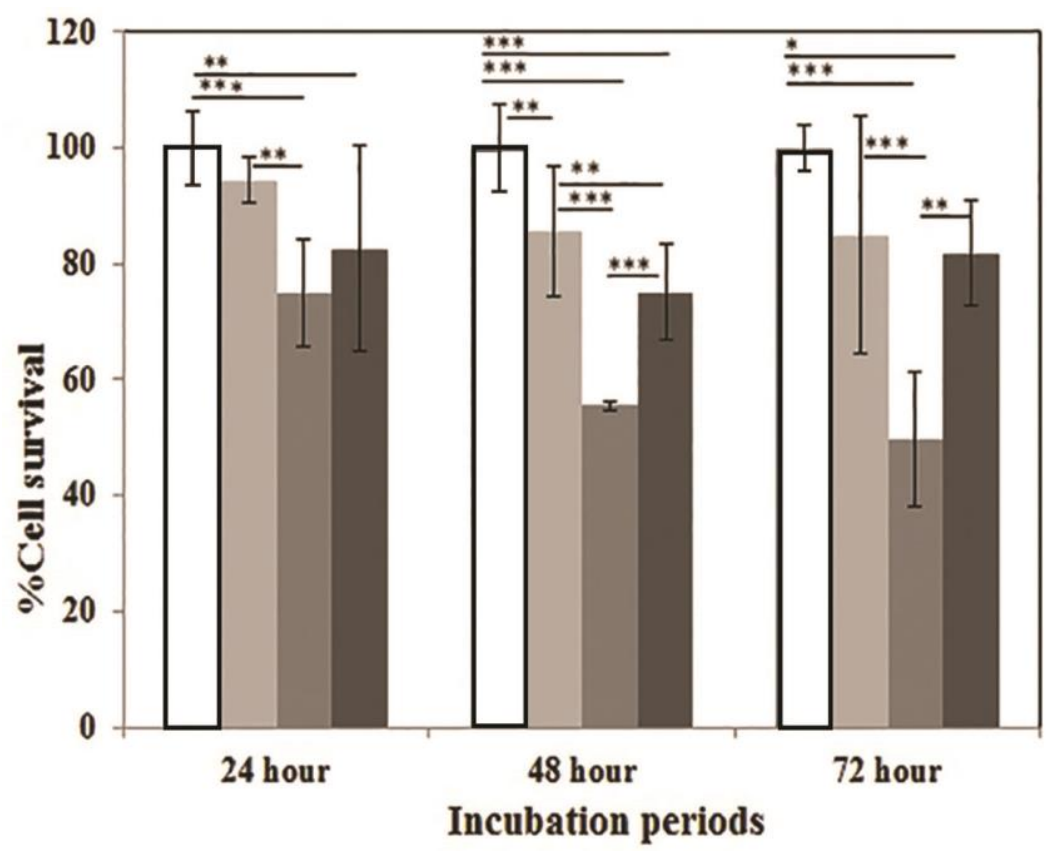

Figure 47. Effect of neat and nylon coated UHMWPE extracts on the viability of L929 fibroblast cells at 24, 48, and $72 \mathrm{~h}\left({ }^{*} \mathrm{p}<0.05\right.$, ** $\mathrm{p}<0.01$, and $\left.* * * \mathrm{p}<0.001\right): \square$, Cell+fresh medium; $\square$, Cells+shaked medium; $\square$, Neat UHMWPE; $\mathbf{\square}$, Nylon 6,6 coated UHMWPE.

The morphology of L929 fibroblasts upon exposure to three conditions, i.e. control (cells+fresh medium), neat UHMWPE, and Nylon 6,6 coated UHMWPE, are depicted in Figure 48 for a visual comparison of the cell viability. The effect of conditioned medium on the rate of cell division was observed throughout three incubation periods (i.e. 24, 48, and 72 h). From Figure 48, it was evident that the cell density (i.e. the density of the viable cells) in cell culture plate upon exposure to a medium conditioned with neat nylon is less than that observed in a medium conditioned with Nylon 6,6 coated UHMWPE at 48 and $72 \mathrm{~h}$ incubation times.

At $72 \mathrm{~h}$, it is shown that most of the cells in the culture plate exposed to the medium conditioned with nylon coated UHMWPE extract could adopt the fibroblast elongated morphology with insignificant clustering structure. On the other hand, many of the cells attached in the culture 
plate exposed to the medium conditioned with uncoated UHMWPE extract could maintain their spherical shapes and showed a more clear evidence of clustered structure, which represents the lower cell viability (i.e. coagulated and detached from the surface of the membrane to die).

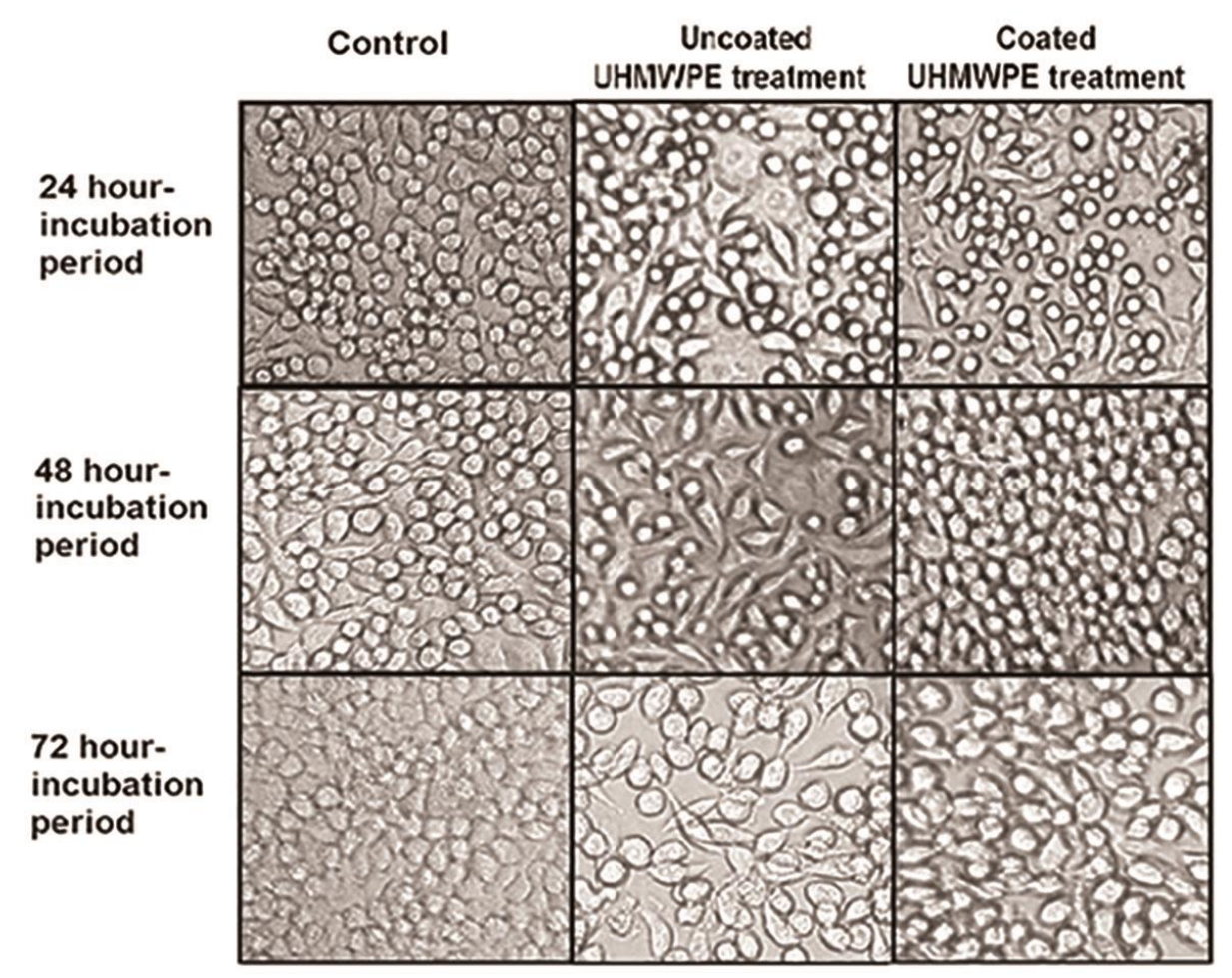

Figure 48. Magnified ( $\times 200)$ microscopic images of the cell viability of L929 fibroblasts upon exposure to three conditions, i.e. control (normal fresh medium+cells), neat UHMWPE, and Nylon 6,6 coated UHMWPE, at three incubation periods $(24,48$, and $72 \mathrm{~h})$.

\subsubsection{Osteolysis and Apoptosis Results}

The osteolysis results after exposing U-937 human macrophage cells in the conditioned media of neat and Nylon 6,6 coated UHMWPE material was compared with the control for three incubation periods $(24,48$, and $72 \mathrm{~h})$ and depicted in Figure 49 and Figure 50. From the results, the expression level of TNFa in the unconditioned medium was nearly double of its level in the media conditioned with neat or Nylon 6,6 coated UHMWPE materials in the case of $24 \mathrm{~h}$ incubation period $(\mathrm{p}<0.01)$. In the case of $48 \mathrm{~h}$ incubation period, the expression level of TNF $\alpha$ 
in the medium conditioned with neat UHMWPE was about 2.5 fold more than the expression level of TNF $\alpha$ in Nylon 6,6 coated material $(\mathrm{p}<0.01$ ). The expression level of TNF $\alpha$ in the medium conditioned with Nylon 6,6 coated UHMWPE was even less than the expression level of TNF $\alpha$ in the unconditioned medium $(\mathrm{p}<0.05)$. In the case of $72 \mathrm{~h}$ incubation period, no significant difference was observed in the expression level of TNF $\alpha$ in all three conditions.

Also, no significant difference was observed in the expression level of IL-6 in all three conditions except in the case of $72 \mathrm{~h}$ incubation period, where the expression level of IL- 6 in macrophages cultured in the unconditioned medium and in the medium conditioned with uncoated UHMWPE material was about threefold more than the expression level of IL-6 in the medium conditioned with Nylon 6,6 coated UHMWPE material ( $\mathrm{p}<0.01)$. The Nylon 6,6 coated UHMWPE material showed a statistically significant lower level of expression of IL-6 from the control $(\mathrm{p}<0.05)$

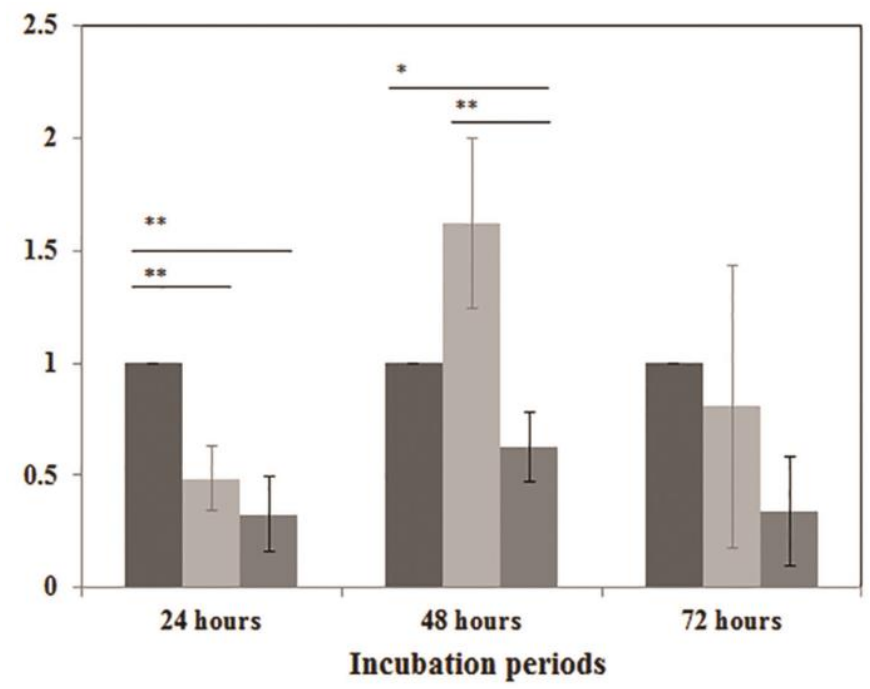

Figure 49. Expression levels of TNF $\alpha$ marker at 24,48 , and 72 incubation periods $\left({ }^{*} \mathrm{p}<0.05\right.$, **p < 0.01, and $* * *$ p < 0.001): $\mathbf{\square}$, Cell+fresh medium; $\square$, Neat UHMWPE; $\square$, Nylon 6,6 coated UHMWPE. 


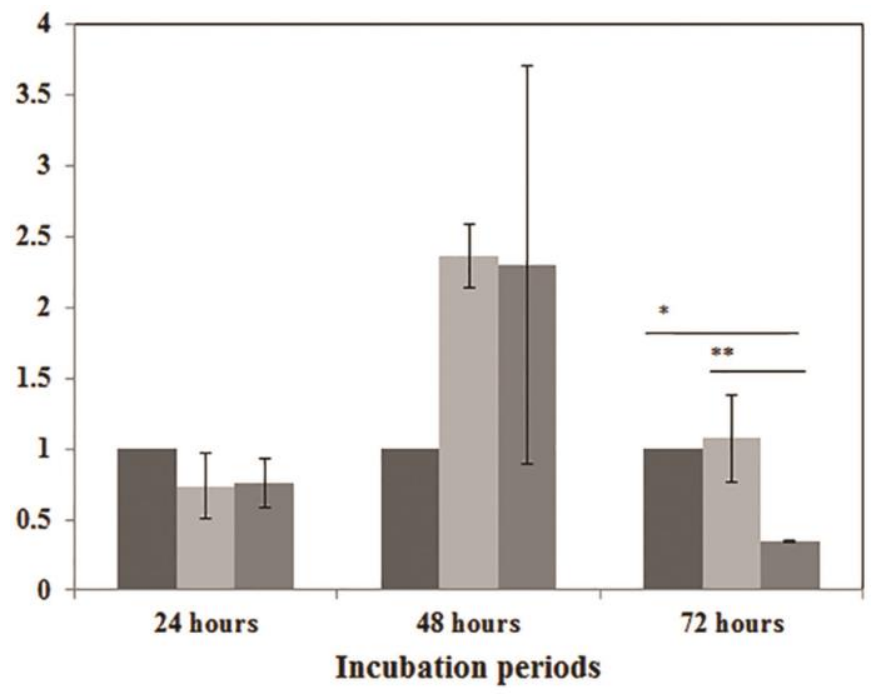

Figure 50. Expression levels of IL-6 marker at 24, 48, and $72 \mathrm{~h}$ incubation periods (*p $<0.05$, **p < 0.01 , and $* *$ p < 0.001): $\mathbf{\square}$, Cell+fresh medium; $\square$, Neat UHMWPE; $\square$, Nylon 6,6 coated UHMWPE. The apoptosis results after exposing L929 fibroblast cells in the conditioned media of neat and Nylon 6,6 coated UHMWPE material was compared with the control for three incubation periods $(24,48$, and $72 \mathrm{~h}$ ) and depicted in Figure 51 and Figure 52 (See Appendix A.1). From the results, no significant difference was detected in the expression levels of Bax and Caspas3, which means that nylon coated material did not induce more apoptosis compared with the neat UHMWPE. Further investigations are required to study the mechanism and pathway of the cell death by examining other apoptotic markers or via other techniques of apoptosis assay.

\subsubsection{Summary}

UHMWPE has come forward as a good material candidate that provides most of the mechanical and biological requirements for joint replacement implants. However, there are some important shortfalls with UHMWPE that could limit the longevity of the final implants, such as the generation of wear particles and moderate creep resistance (see Section 5.1). In this study, it was proposed that coating UHMWPE with nylon could provide superior mechanical properties and better in vitro biocompatibility compared to neat UHMWPE. As it was mentioned previously in 
Section 4.1, nylon coated UHMWPE fibre had lower tensile modulus, higher creep resistance, higher tensile strength, and higher toughness than neat UHMWPE fibre.

Despite the competent mechanical properties and the biocompatibility of UHMWPE in physiological environment, UHMWPE could be toxic to cells in culture as rat tail fibroblasts grown on UHMWPE surfaces synthesized significantly less DNA than cells on other orthopedic biomaterials [158]. In general, while biomaterials are considered insoluble, chemical components could leach out of implants [159]. For instance, direct evidence of the existence of chemical eluates from UHMWPE, which were toxic to cells in culture, was previously found. It was then concluded that the leachable eluates from UHMWPE implants contributed to the osteolytic process at bone-implant interfaces [152].

A time-dependent cell growth inhibition of L929 cells, upon the exposure to the media conditioned with neat UHMWPE, was observed in this study. At the concentration tested, no significant cell death was observed in the case of the medium conditioned with Nylon 6,6 coated UHMWPE while the morphology of cells appeared normal. These results were in the agreement with in vitro results from the literature which reported that UHMWPE particles inhibited: (i) Human osteoblastic cell growth $[160,161]$ and (ii) Macrophage cell growth in culture $[162,163]$. In general, osteolysis, which refers to bone resorption, results to implant failure [152]. It was reported that wear particles, leached out of an artificial joint, can trigger an autoimmune response at the site of transplantation resulting in the loosening of the prosthesis. The molecular mechanisms leading to osteolysis include the upregulation of cytokines, such as IL-6 and TNF $\alpha$ [164-167]. It was also reported that ablating the function of TNF $\alpha$ in mice led to protection from bone loss [168]. Moreover, the role of IL-6 in bone resorption in post-menopausal osteoporosis 
has been well established [169] and it was shown that IL-6 promoted osteolysis by affecting osteoclast differentiation and activity [170].

From the results, the release of pro-inflammatory cytokines IL-6 and TNF $\alpha$ was elevated when human macrophage (U-937) cells were grown in the medium conditioned with neat UHMWPE more than those released by cells grown in the medium conditioned with Nylon 6,6 coated UHMWPE or regular unconditioned medium (See Section 5.3.2). These results are in agreement with clinical data reporting the elevated serum levels of IL-6 in patients with loosened hip prostheses [143]. 


\section{CHAPTER 6 CONCLUSIONS AND FUTURE WORK}

This research was initiated to develop new composite materials for enhancing body protection based on ultra high molecular weight polyethylene (UHMWPE) and silica-based shear thickening fluid (STF) materials. The initial strategy of this work was to improve the adhesion of inert UHMWPE polymer and to evaluate the performance of the developed composite materials. A new approach for UHMWPE surface modification was developed whereby nylon materials were coated on to UHMWPE fibre to improve its overall physical properties. Using an automated coating system exclusively made for this work, Nylon 6,6 and Nylon 6,12 were successfully coated onto the UHMWPE fibre. A 5 wt. \% nylon solution was prepared by dissolving nylon in refluxing alcohol in the presence of calcium chloride. When UHMWPE fibre passed through the solution and then through a water washing step to reprecipitate the nylon, a mechanical interlocking mechanism where the nylon contracted tightly and uniformly around the UHMWPE fibre. Neat and nylon coated UHMWPE fabric samples were tested against different weapons to evaluate their penetration resistance according to the standard protocols. A parallel study was conducted to investigate tensile properties and creep resistance of neat and nylon coated fibres at 25,50 , and $70{ }^{\circ} \mathrm{C}$, in order to gain a better understanding of the mechanical properties of nylon coated fibres. The morphology of the failed fibre/fabric samples was analyzed with scanning electron microscope (SEM) to better understand the damage mechanism(s) of the nylon coating. The next stage of this work was to identify and prepare a high performance and stable STF material using fumed silica particles integrated into a multilayer stack of nylon coated UHMWPE fabric for enhanced penetration resistance. The STF materials were prepared through a sequential ultrasound treatment, as traditional techniques to load particles were not applicable for fumed silica. A complete set of rotational and oscillatory 
rheological measurements, zeta potential measurement, and SEM analysis was carried out in order to control the shear thickening strength of the synthesized samples, yet they remained stable. When the synthesized STF material was applied on neat and nylon coated UHMWPE fabric samples, improved adhesion of the STF was only observed with the nylon coating samples.

Future work in our group will be undertaken to evaluate the resistance of STF/nylon/UHMWPE weaves. This composite material where the interstitial sites of the woven individually nylon coated fibres should readily accommodate and support STF. In a continuing effort to further evaluate the mechanical and physical properties of the nylon coated UHMWPE fibre, a potential biomedical application was investigated, which involved positive biological assessments (biocompatibility, cyctoxicty) for nylon coated UHMWPE in correlation with other mechanical properties.

The subsequent sections list the main conclusions of this research, the main contributions of this research, and several recommendations for future work.

\subsection{Conclusions}

The main conclusions that can be summarized from this research are the following points:

- The mechanical interlocking of Nylon 6,6 or Nylon 6,12 solution could create a uniform and stable coating on the inherently inert UHMWPE fibre/fabric as a result of nylon film shrinkage upon removal of $\mathrm{CaCl}_{2}$ and drying without adding extra thickness to the fibre.

- From the quasi-static and dynamic penetration test results on fabric samples, it was found that nylon coated UHMWPE fabric provided enhanced resistance compared to neat UHMWPE fabric, based on the equal areal density of the samples compared. Also, it was noted that nylon coated UHMWPE fabric samples were affected less by immersion in water, 
in regard to the penetration resistance of the samples, when compared with the neat UHMWPE fabric sample.

- From the tensile and creep test results on fibre samples, it was found that, in general, nylon coated UHMWPE fibre had lower tensile modulus, higher creep resistance, higher tensile strength, and higher toughness than neat UHMWPE fibre at 25,50 , and $70{ }^{\circ} \mathrm{C}$ without compromising the elongation. The results from the static and dynamic penetration tests were then correlated to the observed microscopic damage mechanisms.

- A controlled time of high-intensity ultrasound treatment could control the rheological characteristics and stability of fumed silica dispersion in PEG with long-lasting effects. For instance, the sample with a lower concentration of fumed silica particles or longer time period of ultrasonication exhibited a lower maximum shear thickened viscosity (but a higher critical shear rate) and more viscous-like behaviour over a greater domain of angular frequencies or strain amplitudes, and possessed a lower flow stress (showing a viscous response) under dynamic shear. Also, fumed silica dispersion showed a more stable state at a range of temperature from $25{ }^{\circ} \mathrm{C}$ to $85^{\circ} \mathrm{C}$.

- It was also concluded that the impact of ultrasonication was not due to the change of electrostatic interactions between particles as zeta potential did not change in this treatment. Therefore, the impact of ultrasonication to alter the viscoelastic characteristics of STF samples was correlated to the weakening of hydrogen bonding but not the changes of electrostatic repulsion forces.

- From the SEM images of neat and nylon coated UHMWPE fabrics impregnated with the synthesized shear thickening fluid (STF) material, it was found that the adhesion of STF material to the nylon coated UHMWPE fabric sample is more noticeable when compared 
with the neat UHMWPE fabric sample, which was correlated to the presence of amide linkages in the structure of polyamide nylons.

- From the biological test results which were performed in a continuing effort to evaluate the mechanical and physical properties of nylon coated UHMWPE, in general, it was found that nylon coated UHMWPE showed a more biological compatibility than neat UHMWPE in terms of inducing less osteolysis and inducing less cytotoxicity. Less induced osteolysis by nylon coated UHMWPE compared to neat UHMWPE was then interpreted to be indicative of less wear debris generation.

\subsection{Contributions}

The most important contributions drawn from this research are summarized by the following points:

- A new technique was introduced to increase the mechanical performance of UHMWPE fibre/fabric by coating this polymer with a nylon solution. An automated coating system was manufactured exclusively for this coating process on fibres. It was shown that the mechanical interlock of nylon, facilitates coating of inert UHMWPE fibre/fabric without suffering from poor adhesion to this polymer.

- This is the first study of its kind conducted on UHMWPE fabric with regards to the enhancement of penetration resistance by the means of coating. Similarly, for the first time, tensile and creep tests and SEM analysis were performed on nylon coated UHMWPE fibre to determine its mechanical properties. Also, a better understanding the effect of temperature on the performance of this product was achieved. Different test setups were built according to the standard protocols to perform quasi-static and dynamic penetration tests on fabric samples. 
- A new fabrication technique was developed to make an STF material with a high loading of fumed silica particles, which provides a high shear thickening performance and stability. The long-lasting effect of ultrasonication on the rheological properties of fumed silica dispersion in PEG was then studied in details for the first time. Moreover, it was shown that the nylon coating renders the surface of inert UHMWPE material to a better host for STF materials.

- For a different potential application rather than the manufacturing of protective clothing, this study introduced nylon coated UHMWPE as a potential material to be used for clinical applications.

- A comprehensive review of liquid body armor and the mechanism of penetration resistance by multilayer fabric systems was also presented, which has never been achieved to this extent.

\subsection{Future Work}

In addition to the contributions which have been already made in this study, there are several suggestions that can be performed to further advance this research topic. The following points are a summary of recommendations for future work:

- A similar experimental test study could be designed to evaluate the penetration resistance of a possibly more efficient nylon coated UHMWPE fabric: to coat individual UHMWPE fibres prior to the creation of weaved fabric. This other procedure may also provide a better coating surface onto fabric: the holes between adjacent filaments will not be covered with the nylon coating, in contrast with the current products, while the wettability of the coated fabric will presumably improve. In addition, the nylon coated fibre could be weaved to construct various structures of fabrics to examine flexibility and performance in more detail. 
- The effectiveness of a nylon coating to facilitate adhesion between inert UHMWPE fabric and such an STF material will be evaluated, with regards to the penetration resistance of the STF-nylon-textile system. In case that the chain-like structure of fumed slica particles is a barrier to withstand against high impact energies, other STF materials made of differenet silica particles may be examined to evaluate the performance of such a system. 


\section{APPENDICES}

\section{A.1 Apoptosis Assay Results}

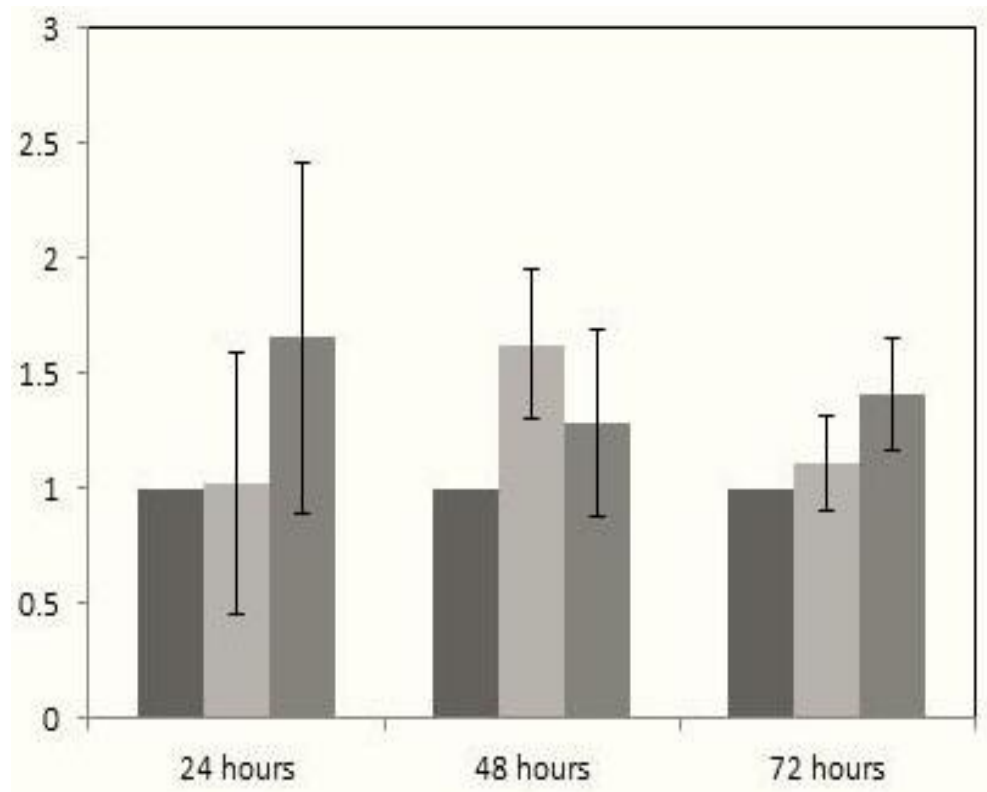

Figure 51. Expression levels of Bax marker at 24, 48, and $72 \mathrm{~h}$ incubation periods: $\mathbf{a}$, Cell+fresh medium; , Neat UHMWPE; - Nylon 6,6 coated UHMWPE.

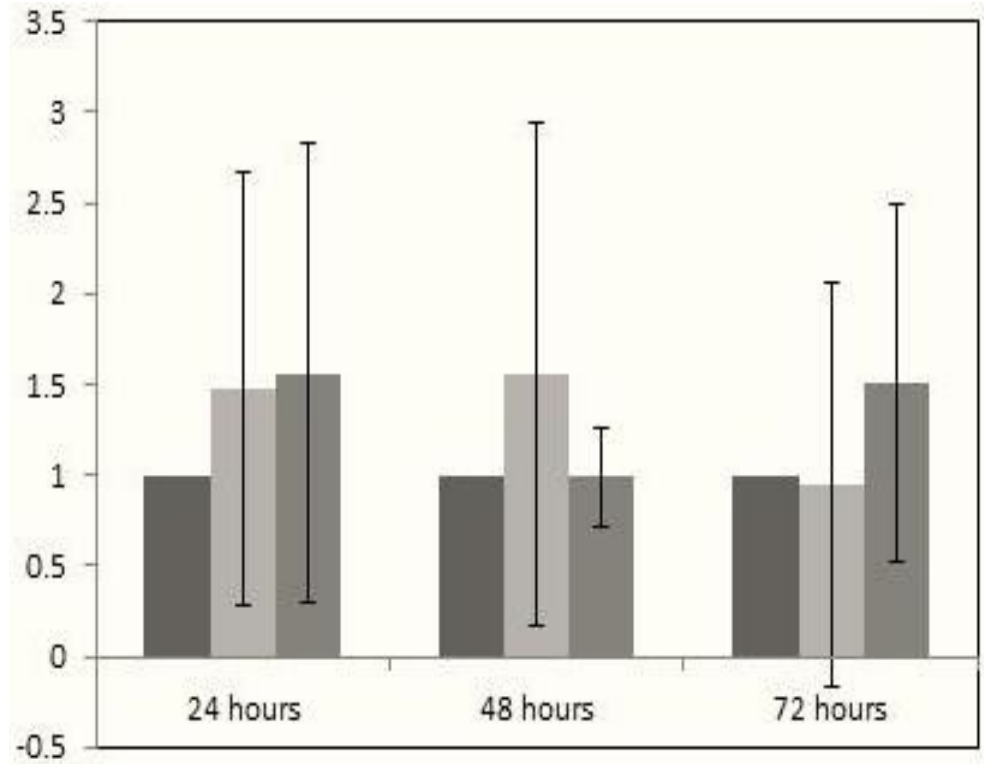

Figure 52. Expression levels of Caspase 3 marker at 24, 48, and $72 \mathrm{~h}$ incubation periods: $\mathbf{\square}$, Cell+fresh medium; $\square$, Neat UHMWPE; $\square$, Nylon 6,6 coated UHMWPE. 


\section{REFERENCES}

1. Egres, R.G., Decker, M.J., Halbach, Y.S., Kirkwood, J.E., Kirkwood, K.M., Wagner, N.J., Wetzel, E.D.: Stab Resistance of Shear Thickening Fluid (STF)-Kevlar Composites for Body Armor Applications. Proceedings of the 24th Army Science Conference, November 29- December 2, Orlando, United States (2004).

2. Egres, R.G., Lee, Y.S., Kirkwood, J.E., Wetzel, E.D., Wagner, N.J.: Liquid armor: Protective Fabrics Utilizing Shear Thickening Fluids. Proceedings of the International Conference on Safety and Protective Fabrics, October 26-27, Pittsburgh, United States (2004).

3. Decker, M.J., Halbach, C.J., Nam, C.H., Wagner, N.J., Wetzel, E.D.: Stab Resistance of Shear Thickening Fluid (STF)-Treated Fabrics. Composites Sci. Technol. 67, 565-578 (2007).

4. Flambard, X., Polo, J.: Stab Resistance of Multi-Layers Knitted Structures (Comparison Between ParaAramid and PBO Fibers). J. Adv. Mater. 36, 30-35 (2004).

5. Mayo Jr., J.B., Wetzel, E.D., Hosur, M.V., Jeelani, S.: Stab and Puncture Characterization of Thermoplastic-Impregnated Aramid Fabrics. Int. J. Impact. Eng. 36, 1095-1105 (2009).

6. Dauvergne, M.: Knives and violent crime in Canada. Statistics Canada, http://www.statcan.gc.ca/pub/85-002-x/2010001/article/11146-eng.htm (2008).

7. Gadow, R., Von Niessen, K.: Lightweight Ballistic Structures Made of Ceramic and Cermet/Aramid Composites. Ceram. Trans. 151, 3-18 (2003).

8. Hosur, M.V., Mayo Jr., J.B., Wetzel, E., Jeelani, S.: Studies on the Fabrication and Stab Resistance Characterization of Novel Thermoplastic-Kevlar Composites. Solid State Phenom. 136, 83-92 (2008).

9. Majumdar, A., Butola, B.S., Srivastava, A.: Development of Soft Composite Materials With Improved Impact Resistance Using Kevlar Fabric and Nano-Silica Based Shear Thickening Gluid. Mater. Design. 54, 295-300 (2014).

10. Lee, Y.S., Wetzel, E.D., Wagner, N.J.: The Ballistic Impact Characteristics of Kevlar Woven Fabrics Impregnated With A Colloidal Shear Thickening Fluid. J. Mater. Sci. 38, 2825-2833 (2003).

11. Tan, V.B.C., Tay, T.E., Teo, W.K.: Strengthening Fabric Armour with Silica Colloidal Suspensions. Int. J. Solids. Struct. 42, 1561-1576 (2005).

12. Erlich, D.C., Shockey, D.A., Simons, J.W.: Slow Penetration of Ballistic Fabrics. Text. Res. J. 73, 179-184 (2003).

13. Carr, D.J.: Failure Mechanisms of Yarns Subjected to Ballistic Impact. J. Mater. Sci. Lett. 18, 585588 (1999).

14. DuPont ${ }^{\mathrm{TM}}$, Technical Guide for Kevlar ${ }^{\circledR}$ aramid fiber, H-77848 4/00.

15. Ding, J., Li, W., Shen, S.Z.: Research and Applications of Shear Thickening Fluids. Recent Pat. Mater. Sci. 4, 43-49 (2011). 
16. Srivastava, A., Majumdar, A., Butola, B.S.: Improving the Impact Resistance Performance of Kevlar Fabrics Using Silica Based Shear Thickening Fluid. Mat. Sci. Eng. A. 529, 224-229 (2011).

17. Majumdar, A., Butola, B.S., Srivastava, A.: An Analysis of Deformation and Energy Absorption Modes of Shear Thickening Fluid Treated Kevlar Fabrics as Soft Body Armour Materials. Mater. Des. 51, 148-153 (2013).

18. Egres Jr, R.G., Lee, Y.S., Kirkwood, J.E., Kirkwood, K.M., Wetzel, E.D., Wagner, N.J.: Advanced Body Armor Utilizing Shear Thickening Fluids. Proceedings of the 23rd Army Science Conference, December 2-5, Orlando, United States (2002).

19. Song, Z., Zhang, C., Song, M., Wu, S.: Advanced Stab Resistance fabrics utilizing shear thickening fluids. Adv. Mat. Res. 299-300, 73-76 (2011).

20. Zhao, S., Zhang, M., Ren, Y., Yang, W., Wu, S.: Impact Resistance of Shear Thickening Fluid (STF)/Kevlar Composites for Body Armor Applications. Adv. Mat. Res. 834-836, 241-245 (2014).

21. Wetzel, E.D., Lee, Y.S., Egres, R.G., Kirkwood, K.M., Kirkwood, J.E., Wagner, N.J.: The Effect of Rheological Parameters on the Ballistic Properties of Shear Thickening Fluid (STF)-Kevlar Composites. Proceedings of the 8th International Conference on Numerical Methods in Industrial Forming Processes. , June 13-17, Columbus, United States (2004).

22. Kalman, D.P., Schein, J.B., Houghton, J.M., Laufer, C.H.N., Wetzel, E.D., Wagner, N.J.: Polymer Dispersion Based Shear Thickening Fluid-Fabrics for Protective Applications. Proceedings of SAMPE 2007, June 3-7, Baltimore, Maryland (2007).

23. Kalman, D.P., Merrill, R.L., Wagner, N.J., Wetzel, E.D.: Sources of Nanoparticle Improvement to Fabric Composite Properties: Shear-Thickening Fluids and Particle Hardness. Proceedings of the American Society for Composites-24th Technical Conference, September 15-17, Wilmington, United States (2009).

24. Rao, H., Hosur, M.V., Mayo Jr, J., Burton, S., Jeelani, S.: Stab Characterization of Hybrid Ballistic Fabrics. Proceedings of the SEM Annual Conference, June 1-4, Albuquerque, United States (2009).

25. Rao, H.M., Mayo Jr, J., Hosur, M.V., Jeelani, S.: Stab Characterization of Ballistic Fabrics Impregnated With Shear Thickening Fluid. Proceedings of the International SAMPE Technical Conference, September 8-11, Memphis, United States (2008).

26. Kang, T.J., Hong, K.H., Yoo, M.R.: Preparation and Properties of Fumed Silica/Kevlar Composite Fabrics for Application of Stab Resistant Material. Fiber. Polym. 11, 719-724 (2010).

27. Egres Jr, R.G., Halbach, C.J., Decker, M.J., Wetzel, E.D., Wagger, N.J.: Stab Performance of Shear Thickening Fluid (STF)-Fabric Composites for Body Armor Applications. Proceeding of the International SAMPE Symposium and Exhibition, May 1-5, Long Beach, United States (2005).

28. Ngothai, Y., Donnelly, S., Woolley, M., Nguyen, Q.D.: Investigatio of the Rheologial Characteristics of Shear Thickening Fluids for Potential Applications in Body Armour. Proceeding of the Chemeca 2008 Conference, September 28 - October 1, Newcastle, Australia (2008). 
29. Park, J.L., Yoon, B.L., Paik, J.G., Kang, T.J.: Ballistic Performance of p-Aramid Fabrics Impregnated With Shear Thickening Fluid; Part II - Effect of Fabric Count and Shot Location. Text. Res. J. 82, 542557 (2012).

30. Park, J.L., Yoon, B.L., Paik, J.G., Kang, T.J.: Ballistic Performance of p-Aramid Fabrics Impregnated With Shear Thickening Fluid; Part I - Effect of Laminating Sequence. Text. Res. J. 82, 527-541 (2012).

31. Lin, H.W., Hwu, W.H., Ger, M.D.: The Mechanism of Shear Thickening Fluid With Nanoparticles Applied to Liquid Armor. Proceedings of the 20th International Microprocesses and Nanotechnology Conference, November 5-8, Kyoto, Japan (2007).

32. Rabb, R.J., Fahrenthold, E.P.: Evaluation of Shear-Thickening-Fluid Kevlar for Large-FragmentContainment Applications. J. Aircr. 48, 230-234 (2011).

33. Decker, M.J., Egres, R.G., Wetzel, E., Wagner, N.J.: Low Velocity Ballistic Properties of Shear Thickening Fluid (STF)-Fabric Composites. Proceedings of the 22nd International Symposium on Ballistics, November 14-18, Vancouver, Canada (2005).

34. Kalman, D.P., Merrill, R.L., Wagner, N.J., Wetzel, E.D.: Effect of Particle Hardness on the Penetration Behavior of Fabrics Intercalated With Dry Particles and Concentrated Particle-Fluid Suspensions. ACS Appl. Mater. Interfaces. 1, 2602-2612 (2009).

35. Mahfuz, H., Clements, F., Rangari, V., Dhanak, V., Beamson, G.: Enhanced Stab Resistance of Armor Composites With Functionalized Silica Nanoparticles. J. Appl. Phys. 105, 064307 (2009).

36. Yu, K., Cao, H., Qian, K., Jiang, L., Li, H.: Synthesis and Stab Resistance of Shear Thickening Fluid (STF) Impregnated Glass Fabric Composites. Fibers Text. East. Eur. 20, 126-128 (2012).

37. Rosen, B.A., Laufer, C.H.N., Kalman, D.P., Wetzel, E.D., Wagner, N.J.: Multi-Threat Performance of Kaolin-Based Shear Thickening Fluid (STF)-Treated Fabrics. Proceedings of the International SAMPE Symposium and Exhibition, June 3-7, Baltimore, United States (2007).

38. Hassan, T.A., Rangari, V.K., Jeelani, S.: Synthesis, Processing and Characterization of Shear Thickening Fluid (STF) Impregnated Fabric Composites. Mat. Sci. Eng. A-Struct. 527, 2892-2899 (2010).

39. Sun, L.L., Xiong, D.S., Xu, C.Y.: Application of Shear Thickening Fluid in Ultra High Molecular Weight Polyethylene Fabric. J. Appl. Polym. Sci. 129, 1922-1928 (2013).

40. Lane, R.A.: High Performance Fibers for Personnel and Vehicle Armor Systems. The AMPTIAC Quarterly, Volume 9, Number 2, https://www.dsiac.org/sites/default/files/AmmtiacJournals/AMPQ9_2.pdf (2005).

41. Purushothaman, A., Coimbatore, G., Ramkumar, S.S.: Soft Body Armor for Law Enforcement Applications. J. Eng. Fiber Fabr. 8, 97-103 (2013).

42. Shockey, D.A., Erlich, D.C., Simons, J.W.: Lightweight Fragment Barriers for Commercial Aircraft. Proceedings of the 18th International Symposium on Ballistics, November 15-19, San Antonio, United States (1999). 
43. Kurtz, S.M.: UHMWPE Biomaterials Handbook - 2nd. Elsevier Inc., Burlington, USA (2009).

44. Lu, S.H., Liang, G.Z., Zhou, Z.W., Li, F.: Structure and Properties of UHMWPE Fiber/Carbon Fiber Hybrid Composites. J. Appl. Polym. Sci. 101, 1880-1884 (2006).

45. Debnath, S., Ranade, R., Wunder, S.L., Baran, G.R., Zhang, J.M., Fisher, E.R.: Chemical Surface Treatment of Ultrahigh Molecular Weight Polyethylene for Improved Adhesion to Methacrylate Resins. J. Appl. Polym. Sci. 96, 1564-1572 (2005).

46. Hofsté, J.M., Schut, J.A., Pennings, A.J.: The Effect of Chromic Acid Treatment on the Mechanical and Tribological Properties of Aramid Fibre Reinforced Ultra-High Molecular Weight Polyethylene Composite. J. Mater. Sci. Mater. Med. 9, 561-566 (1998).

47. Cheeseman, B.A., Bogetti, T.A.: Ballistic Impact Into Fabric and Compliant Composite Laminates. Compos. Struct. 61, 161-173 (2003).

48. Kirkwood, J.E., Kirkwood, J.E., Lee, Y.S., Egres Jr, R.G., Wagger, N.J., Wetzel, E.D.: Yarn Pull-Out as a Mechanism for Dissipation of Ballistic Impact Energy in Kevlar KM-2 Fabric; Part II: Predicting Ballistic Performance. Text. Res. J. 74, 938-948 (2004).

49. Kirkwood, K.M., Kirkwood, J.E., Lee, Y.S., Egres Jr, R.G., Wetzel, E.D., Wagner, N.J.: Yarn PullOut as a Mechanism for Dissipation of Ballistic Impact Energy in Kevlar KM-2 Fabric; Part I: QuasiStatic Characterization of Yarn Pull-Out. Text. Res. J. 74, 920-928 (2004).

50. Bazhenov, S.: Dissipation of Energy by Bulletproof Aramid Fabric. J. Mater. Sci. 32, 4167-4173 (1997).

51. Wetzel, E.D., Wagner, N.J.: Comment on "Evaluation of Shear-Thickening-Fluid Kevlar for LargeFragment-Containment Applications". J. Aircr. 49, 671-673 (2012).

52. Gong, X., Xu, Y., Zhu, W., Xuan, S., Jiang, W., Jiamg, W.: Study of the Knife Stab and PunctureResistant Performance for Shear Thickening Fluid Enhanced Fabric. J. Compos. Mater. 48, 641-657 (2013).

53. Lee, B., Kim, I., Kim, C.: The Influence of the Particle Size of Silica on the Ballistic Performance of Fabrics Impregnated With Silica Colloidal Suspension. J. Compos. Mater. 43, 2679-2698 (2009).

54. Naik, N.K., Shrirao, P., Reddy, B.C.K.: Ballistic Impact Behaviour of Woven Fabric Composites: Formulation. Int. J. Impact. Eng. 32, 1521-1552 (2006).

55. Ahmad, M.R., Ahmad, W.Y.W., Salleh, J., Samsuri, A.: Effect of Fabric Stitching on Ballistic Impact Resistance of Natural Rubber Coated Fabric Systems. Mater. Des. 29, 1353-1358 (2008).

56. Abu Bakar, N.A., Salleh, J., Ahmad, M.R., Ahmad, N.A., Ahmad Suhaimi, S.: Puncture Resistance of Nanofilled Coated High Strength Woven Fabric. Proceedings of the IEEE Symposium on Business, Engineering and Industrial Applications, September 23-26, Bandung, Indonesia (2012).

57. Hassim, N., Ahmad, M.R., Wan, A., W.Y.W., Samsuri, A., Yahya, M.H.M.: Puncture Resistance of Natural Rubber Latex Unidirectional Coated Fabrics. J. Ind. Text. 42, 118-131 (2011). 
58. Ahmad, M.R., Ahmad, W.Y.W., Salleh, J., Samsuri, A.: Performance of Natural Rubber Coated Fabrics Under Ballistic Impact. MPJ. 2, 39-51 (2007).

59. Ahmad, M.R., Ahmad, W.Y.W., Samsuri, A., Salleh, J.: Ballistic Response of Natural Rubber Latex Coated and Uncoated Fabric Systems. J. Rubb. Res. 10, 207-221 (2007).

60. Firouzi, D., Foucher, D.A., Bougherara, H.: Nylon Coated UHMWPE Fibers With Improved Properties and Apparatus for Coating. US Patent Application No.: 13/858,705 (2012).

61. Firouzi, D., Youssef, A., Amer, M., Srouji, R., Amleh, A., Foucher, D.A., Bougherara, H.: A New Technique to Improve the Mechanical and Biological Performance of Ultra High Molecular Weight Polyethylene Using A Nylon Coating. J. Mech. Behav. Biomed. Mater. 32, 198-209 (2014).

62. Firouzi, D., Foucher, D.A., Bougherara, H.: Nylon-Coated Ultra High Molecular Weight Polyethylene Fabric for Enhanced Penetration Resistance. J. Appl. Polym. Sci. 131, 40350 (2014).

63. Glaser, R., Caccese, V.: Experimental Methods to Determine In-Plane Material Properties of Polyurethane-Coated Nylon Fabric. J. Text. Inst. 104, 682-698 (2013).

64. Zeng, X.S., Shim, V.P.W., Tan, V.B.C.: Influence of Boundary Conditions on the Ballistic Performance of High-Strength Fabric Targets. Int. J. Impact. Eng. 32, 631-642 (2005).

65. Mouritz, A.P.: Ballistic Impact of Explosive Blast Resistance of Stitched Composites. Compos. Part A. 32, 431-439 (2001).

66. Karahan, M., Kus, A., Eren, R.: An Investigation into Ballistic Performance and Energy Absorption Capabilities of Woven Aramid Fabrics. Int. J. Impact. Eng. 35, 499-510 (2008).

67. Zivkovic, I., Perisic, P., Burzic, Z., Uskokovic, P., Aleksic, R.: Aramid Fiber Reinforced Laminar Thermoplastic Composite Materials. J. Adv. Mater. 37, 23-31 (2005).

68. Liu, S., Wang, J., Wang, Y., Wang, Y.: Improving the Ballistic Performance of Ultra High Molecular Weight Polyethylene Fiber Reinforced Composites Using Conch Particles. Mater. Design. 31, 1711-1715 (2010).

69. Hoffman, R.L.: Discontinuous and Dilatant Viscosity Behavior in Concentrated Suspensions. I. Observation of a Flow Instability. Trans. Soc. Rheol. 16, 155-173 (1972).

70. Hoffman, R.L.: Discontinuous and Dilatant Viscosity Behavior in Concentrated Suspensions. II. Theory and Experimental Tests. J. Colloid Interface Sci. 46, 491-506 (1974).

71. Hoffman, R.L.: Discontinuous and Dilatant Viscosity Behavior in Concentrated Suspensions. III. Necessary Conditions for their Occurrence in Viscometric Flows. Adv. Colloid Interface Sci. 17, 161-184 (1982).

72. Hoffman, R.L.: Explanations for the Cause of Shear Thickening in Concentrated Colloidal Dispersions. J. Rheol. 42, 111-123 (1998). 
73. S.R. Raghavan, S.A.K.: Shear-Thickening Response of Fumed Silica Suspensions Under Steady and Oscillatory Shear. J. Colloid Interface Sci. 185, 57-67 (1997).

74. B.J. Maranzano, N.J. Wagner: The Effect of Interparticle Interactions and Particle Size on Reverse Shear Thickening; Hard-Sphere Colloidal Dispersions. J. Rheol. 45, 1205-1222 (2001).

75. Maranzano, B.J., Wagner, N.J.: The Effects of Particle Size on Reversible Shear Thickening of Concentrated Colloidal Dispersions. J. Chem. Phys. 114, 10514-10527 (2001).

76. Hassan, T.A., Rangari, V.K., Jeelani, S.,: Sonochemical Synthesis and Rheological Properties of Shear Thickening Silica Dispersions. Ultrason. Sonochem. 17, 947-952 (2010).

77. So, J., Yang, S., Hyun, J.: Microstructure Evolution and Rheological Responses of Hard Sphere Suspensions. Chem. Eng. Sci. 56, 2967-2977 (2001).

78.Ye, F., Zhu, W., Jiang, W., Wang, Z., Chen, Q., Gong, X., Xuan, S.: Influence of Surfactants on Shear-Thickening Behavior in Concentrated Polymer Dispersions. J. Nanopart. Res. 15, 2122 (2012).

79. Maranzano, B.J., Wagner, N.J.: Flow-Small Angle Neutron Scattering Measurements of Colloidal Dispersion Microstructure Evolution Through the Shear Thickening Transition. J. Chem. Phys. 117, 10291-10302 (2002).

80. Melrose, J.R., Ball, R.C.: Continuous Shear Thickening Transitions in Model Concentrated Colloids The Role of Interparticle Forces. J. Rheol. 48, 937-960 (2004).

81. Boersma, W.H., Laven, J., Stein, H.N.: Computer Simulation of Shear Thickening if Concentrated Dispersion. J. Rheol. 39, 841-860 (1995).

82. Boersma, W.H., Laven, J., Stein, H.N.: Viscoelastic Properties of Concentrated Shear-Thickening Dispersions. J. Colloid Interface Sci. 149, 10-22 (1992).

83. Catherall, A.A., Melrose, J.R., Ball, R.C.: Shear Thickening and Order-Disorder Effects in Concentrated Colloids at High Shear Rates. J. Rheol. 44, 1-25 (2000).

84. Raghavan, S.R., Khana, S.A.: Shear-Induced Microstructural Changes in Flocculated Suspensions of Fumed Silica. J. Rheol. 39, 1311-1325 (1995).

85. Mewis, J., Wagner, N.J.: Colloidal Suspension Rheology - 1st. Cambridge University Press, Cambridge, USA (2012).

86. Wagner, N.J., Brady, J.F.: Shear Thickening in Colloidal Dispersions. Phys. Today. 62, 27-32 (2009).

87. Cohen, D.: Shear-Thickening Fluid Reinforced Fabrics for Use with an Expandable Spacecraft. US Patent Application No.: 11/807,830 (2008).

88. Bohannan, A.l., Fahrenthold, E.P.: Hypervelocity Impact Simulation Using Membrane ParticleElements. Int. J. Impact. Eng. 32, 1497-1502 (2008). 
89. Bohannan, A.L., Fahrenthold, E.P.: Simulation of STF Kevlar Shielding Performance in a Stuffed Whipple Configuration. Proceedings of the 50th AIAA/ASME/ASCE/AHS/ASC Structures, Structural Dynamics and Materials Conference, May 4-7, Palm Springs, United States (2009).

90. Laun, H.M., Bung, R., Schmidt, F.: Rheology of Extremely Shear Thickening Polymer Dispersions (Passively Viscosity Switching Fluids). J. Rheol. 35, 999-1034 (1991).

91. Zhang, X.Z., Li, W.H., Gong, X.L.: The Rheology of Shear Thickening Fluid (STF) and the Dynamic Performance of an STF-Filled Damper. Smart. Mater. Struct. 17, 035027 (2008).

92. Fischer, C., Braun, S.A., Bourban, P.E., Michaud, V., Plummer, C.J.G., Manson, J.A.E.: Dynamic Properties of Sandwich Structures with Integrated Shear-Thickening Fluids. Smart Mater. Stuct. 15, 1467-1475 (2006).

93. Allen, S.D.: Body Limb Movement Limiter. US Patent Application No.: 11/099,381 (2008).

94. Choi, H.N., Hong, T.M., Lee, E.H., Paik, J.G., Yoon, B.I., Lee, S.G.: Stab Resistance of Aramid Fabrics Reinforced with Silica STF. Proceedings of the18th International Conference on Composites Materials, August 21-26, Jeju, South Korea (2011).

95. Borella, R.P., Barry, R.P., Allgeuer, T.T., Dan Hartog, H.: Plastic Hollow Fibre Containing a Shear Thickening Fluid for High Tensile Strength Fibres. US Patent Application No.: PCT/US2008/053860 (2008).

96. Kalman, D.P., Wagner, N.J.: Microstructure of Shear-Thickening Concentrated Suspensions Determined by Flow-USANS. Rheol. Acta. 48, 897-908 (2009).

97. Nam, C.H., Decker, M.J., Halbach, C., Wetzel, E.D., Wagner, N.J.: Ballistic and Rheological Properties of STFs Reinforced by Short Discontinuous Fibers. Proceedings of the International SAMPE Symposium and Exhibition, May 1-5, Long Beach, United States (2005).

98. Wagner, N.J., Wetzel, E.D.: Comfortable Ballistic Resistant and Protective Composite Materials Composed of Shear Thickening Fluids Reinforced by Fillers Such as Fibers. US Patent Application No.: 60/651,417 (2001).

99. J. Bender, N.J. Wagner: Reversible Shear Thickening in Monodisperse and Bidisperse Colloidal Dispersions. J. Rheol. 40, 899-916 (1996).

100. Amiri, A., Oye, G., Sjoblom, J.: Influence of pH, High Salinity and Particle Concentraion on Stability and Rheological Properties of Aqueous Suspensions of Fumed Silica. Colloids Surf. A Physicochem. Eng. Asp. 349, 43-54 (2009).

101. Amiri, A., Oye, G., Sjoblom, J.: Temperature and Pressure Effects on Stability and Gelation Properties of Silica Properties. Colloids Surf. A Physicochem. Eng. Asp. 378, 14-21 (2011).

102. Smith, W.E., Zukoski, C.F.: Aggregation and Gelation Kinetics of Fumed Silica-Ethanol Suspensions. J. Colloid Interface Sci. 304, 359-369 (2006). 
103. Nordstrom, J., Matic, A., Sun, J., Forsyth, M., MacFarlane, D.R.: Aggregation, Ageing and Transport Properties of Surface Modified fumed Silica Dispersions. Soft Matter. 6, 2293-2299 (2010).

104. Crawford, N.C., Williams, S.K.R., Boldridge, S., Liberatorea, M.W.: Shear Thickening and Defect Formation of Fumed Silica CMP Slurries. Colloids Surf. A Physicochem. Eng. Asp. 436, 87-96 (2013).

105. Khana, S.A., Zoeller, N.J.: Dynamic Rheological Behavior of Flocculated Fumed Silica Suspensions. J. Rheol. 37, 1225-1235 (1993).

106. Raghavan, S.R.: Rheology of Silica Dispersions in Organic Liquids: New Evidence for Solvation Forces Dictated by Hydrogen Bonding. Langmuir. 16, 7920-7930 (2000).

107. Wu, X., Wang, Y., Yang, W., Xie, B., Yang, M., Dan, W.: A Rheological Study on Temperature Dependent Microstructural Changes of Fumed Silica Gels in Dodecane. Soft Matter. 8, 10457-10463 (2012).

108. Wu, X.J., Wang, Y., Wang, M., Yang, W.: Structure of Fumed Silica Gels in Dodecane: Enhanced Network by Oscillatory Shear. Colloid. Polym. Sci. 290, 151-161 (2012).

109. Paquien, J.N., Galy, J., Gerard, J.F., Pouchelon, A.: Rheological Studies of Fumed Silica-

PolydimethylSiloxane Suspensions. Colloids Surf. A Physicochem. Eng. Asp. 260, 165-182 (2005).

110. Ramesh, S., Koltypin, Y., Gedanken, A.: Ultrasound Driven Aggregation and Surface Silanol Modification in Amorphous Silica Microspheres. J. Mater. Res. 12, 3271-3277 (1997).

111. Clements, F.E., Mahfuz, H.: Enhancing the Stab Resistance of Flexible Body Armor Using Functionalized $\mathrm{SiO}_{2}$ Nanoparticles. Proceedings of the ICCM International Conferences on Composite Materials, July 8-13, Kyoto, Japan (2007).

112. Chisholm, N., Mahfuz, H., Rangari, V.K., Ashfaq, A., Jeelani, S.: Fabrication and Mechanical Characterization of Carbon/SiC-Epoxy Nanocomposites. Compos. Struct. 67, 115-124 (2005).

113. Price, G.J.: Recent Developments in Sonochemical Polymerisation. Ultrason. Sonochem. 10, 277283 (2010).

114. Negoro, S.: Biodegradation of Nylon and other Synthetic Polyamides. Biopolymers. 9, 395-415 (2005).

115. M. Sepe, The Materials Analyst, Part 98: A tour of the world of nylon-Part 1. http://www.plasticstoday.com/imm/articles/materials-analyst-part-98-tour-world-nylon151part-1 (2008).

116. NIJ Standard 0115.00: Stab Resistance of Personal Body Armor. National Institute of Justice, USA (2000).

117. ASTM Standard D4833/4833M: Standard Test Method for Index Puncture Resistance of Geomembrances and Related Products. ASTM International, USA (2007).

118. ASTM Standard D2256 / D2256M: Standard Test Method for Tensile Properties of Yarns by the Single-Strand Method. ASTM International, USA (2010). 
119. ASTM Standard D2990: Standard Test Methods for Tensile, Compressive, and Flexural Creep and Creep-Rupture of Plastics. ASTM International, USA (2009).

120. Benhui, S.: Study on the Mechanism of Nylon 6,6 Dissolving Process Using $\mathrm{CaCl}_{2} / \mathrm{MeOH}$ as the Solvent. Chin. J. Polym. Sci. 12, 57-65 (1994).

121. Yang, H.L., Ruan, J.M., Zou, J.P., Wu, Q.M., Zhou, Z.C.: Rheological Response of Fumed Silica Suspensions Under Steady and Oscillatory Shear. Sci. I Chin. Ser. E. 52, 910-915 (2009).

122. Edwards, B.J., Keffer, D.J., Reneau, C.W.: An Examination of the Shear-Thickening Behavior of High Molecular Weight Polymers Dissolved in Low-Viscosity Newtonian Solvents. J. Appl. Polym. Sci. 85, 1714-1735 (2002).

123. Galindo-Rosales, F.J., Rubio-Hernández, F.J., Sevilla, A.: An Apparent Viscosity Function for Shear Thickening Fluids. J. Non-Newton. Fluid. Mech. 166, 321-325 (2011).

124. Mezger, T.G.: The Rheology Handbook: For Users of Rotational and Oscillatory Rheometers - 2nd. Vincentz Network GmbH \& Co., Hannover, Germany (2006).

125. Chang, L., Friedrich, K., Schlarb, A.K., Tanner, R., Ye, L.: Shear-Thickening Behaviour of Concentrated Polymer Dispersions Under Steady and Oscillatory Shear. J. Matter. Sci. 46, 339-346 (2011).

126. Sato, T., Uchida, T., Takeuchi, A.E.S.: Study on dispersion and surface modification of diamond powders by ultrasound exposure. Proceedings of the IEEE Ultrasonics Symposium, October 8-11, (2012).

127. Hasanzadeh, M., Mottaghitalab, V.: The Role of Shear-Thickening Fluids (STFs) in Ballistic and Stab-Resistance Improvement of Flexible Armor. J. Mater. Eng. Perform. 23, 1182-1196 (2014).

128. Yu, K., Cao, H., Qian, K., Sha, X., Chen, Y.: Shear-Thickening Behavior of Modified Silica Nanoparticles in Polyethylene Glycol. J. Nanopart. Res. 14, 747 (2012).

129. Wagger, N.J., Bender, J.W.: The Role of Nanoscale Forces in Colloid Dispersion Rheology. MRS Bulletin. 29, 100-106 (2004).

130. Kang, T.J., Kim, C.Y., Hong, K.H.: Rheological Behavior of Concentrated Silica Suspension and its Application to Soft Armor. J. Appl. Polym. Sci. 124, 1534-1541 (2012).

131. Bougherara, H., Bureau, M.N., Campbell, M., Vadean, A., Yahia, L.: Design of a Biomimetic Polymer-Composite Hip Prosthesis. J. Biomed. Mater. Res. A. 82, 27-40 (2007).

132. Bougherara, H., Bureau, M.N., Yahia, L.: Bone Remodeling in a New Biomimetic PolymerComposite Hip Stem. J. Biomed. Mater. Res. A. 92, 164-174 (2010).

133. Bartel, D.L., Davy, D.T., Keaveny, T.M.: Orthopaedic Biomechanics: Mechanics and Design in Musculoskeletal Systems - 1st Edition. Prentice Hall, New Jersey, USA (2006).

134. Deng, M., Shalaby, S.W.: Properties of Self-Reinforced Ultra-High-Molecular-Weight Polyethylene Composites. Biomaterials. 18, 645-655 (1997). 
135. Shalaby, S.W., Deng, M.: Self-Reinforced Ultra-High Molecular Weight Polyethylene Composites. US Patent Application No.: 08/480,848 (1998).

136. Chang, N., Bellare, A., Cohen, R.A., Spector, M.: Wear Behaviour of Bulk Oriented and Fiber Reinforced UHMWPE. Wear. 241, 109-117 (2000).

137. Xue, Y., Wu, W., Jacobs, O., Schädel, B.: Tribological Behaviour of UHMWPE/HDPE Blends Reinforced with Multi-Wall Carbon Nanotubes. Polym. Test. 25, 221-229 (2006).

138. Xie, X.L., Tang, C.Y., Chan, K.Y., Wu, X.C., Tsui, C.P., Cheung, C.Y.: Wear Performance of Ultrahigh Molecular Weight Polyethylene/quartz Composites. Biomaterials. 24, 1889-1896 (2003).

139. Kang, P.H., Nho, Y.C.: The Effect of $\gamma$-Irradiation on Ultra-High Molecular Weight Polyethylene Recrystallized Under Different Cooling Conditions. Radiat. Phys. Chem. 60, 79-87 (2001).

140. Lewis, G., Carroll, M.: Effect of Crosslinking UHMWPE on its Tensile and Compressive Creep Performance. Bio-Med. Mater. Eng. 11, 167-183 (2001).

141. Mejia, L.C., Brierley, T.J.: A Hip Wear Simulator for the Evaluation of Biomaterials in Hip Arthroplasty Components. Biomed. Mater. Eng. 4, 259-271 (1995).

142. Penmetsa, J.R., Laz, P.J., Petrella, A.J., Rullkoetter, P.J.: Influence of Polyethylene Creep Behavior on Wear in Total Hip Arthroplasty. J. Orthop. Res. 24, 422-427 (2006).

143. Hernigou, P., Poignard, A., Filippini, P., Zilber, S.: Retrieved Unicompartmental Implants with Full PE Tibial Components: The Effects of Knee Alignment and Polyethylene Thickness on Creep and Wear. Open Orthop. J. 2, 51-56 (2008).

144. Lin, S.P., Han, J.L., Yeh, J.T., Chang, F.C., Hsieh, K.H.: Surface Modification and Physical Properties of Various UHMWPE-Fiber-Reinforced Modified Epoxy Composites. J. Appl. Polym. Sci. 104, 655-665 (2007).

145. Zhao, Y., Han, G., Hou, J., Wang, C., Wan, J., Ma, J., Sun, L.: Surface Modification of UHMWPE Fiber by Low Temperature Plasma Treatment and Grafting Using Acrylic Acid. Polym. Mater. Sci. Eng. 29, 62-65 (2013).

146. Morlanes, M.J.M., Castell, P., Nogués, V.M., Martinez, M.T., Alonso, P.J., Puértolas, J.A.: Effects of Gamma-Irradiation on UHMWPE/MWNT Nanocomposites. Composites Sci. Technol. 71, 282-288 (2011).

147. Sreekanth, P.S.R., Kumar, N.N., Kanagaraj, S.: Effect of MWCNT on Mechanical Properties of $\gamma$ Irradiated UHMWPE During Shelf Ageing Process. Adv. Mat. Res. 410, 160-163 (2012).

148. Jia, G., Wang, H., Yan, L., Wang, X., Pei, R., Yan, T., Zhao, Y., Guo, X.: Cytotoxicity of Carbon Nanomaterials: Single-Wall Nanotube, Multi-Wall Nanotube, and Fullerene. Environ. Sci. Technol. 39, 1378-1383 (2005). 
149. Haniu, H., Saito, N., Matsuda, Y., Tsukahara, T., Usui, Y., Narita, N., Hara, K., Aoki, K., Shimizu, M., Ogihara, N., Takanashi, S., Okamoto, M., Kobayashi, S., Ishigaki, N., Nakamura, K., Kato, H.: Basic Potential of Carbon Nanotubes in Tissue Engineering Applications. J. Nanomater. 2012, 1-10 (2012).

150. Pal, S., Roy, S., Bag, S.: Hydroxyapatite Coating over Alumina - Ultra High Molecular Weight Polyethylene Composite Biomaterials. Trends Biomater. Artif. Organs. 18, 106-109 (2005).

151. Silva, M.A., Gomes, P.S., Vila, M., Lopes, M.A., Santos, J.D., Silva, R.F., Fernandes, M.H.: New Titanium and Titanium/Hydroxyapatite Coatings on Ultra-High-Molecular- Weight Polyethylene - In Vitro Osteoblastic Performance. Biomed. Mater. 5, 035014 (2010).

152. Wang, K.Y., Horne, J.G., Devane, P.A., Wilson, T., Miller, J.H.: Chemical Eluates from Ultra-High Molecular Weight Polyethylene and Fibroblast Proliferation. J. Orthop. Surg. (Hong Kong). 9, 25-33 (2001).

153. Trentani, L., Pelillo, F., Pavesi, F.C., Ceciliani, L., Cetta, G., Forlino, A.: Evaluation of the $\mathrm{TiMo}_{12} \mathrm{Zr}_{6} \mathrm{Fe}_{2}$ Alloy for Orthopaedic Implants: In Vitro Biocompatibility Study by Using Primary Human Fibroblasts and Osteoblasts. Biomaterials. 23, 2863-2869 (2002).

154. Kadoya, Y., Kobayashi, A., Ohashi, H.: Wear and Osteolysis in Total Joint Replacements. Acta. Orthop. Scand. Suppl. 278, 1-16 (1998).

155. Jiranek, W.A., Machado, M., Jasty, M., Jevsevar, D., Wolfe, H.J., Goldring, S.R., Goldberg, M.J., Harris, W.H.: Production of Cytokines Around Loosened Cemented Acetabular Components. Analysis With Immunohistochemical Techniques and In Situ Hybridization. J. Bone Joint Surg. Am. 75, 863-879 (1993).

156. Athanasou, N.A., Sabokbar, A.: Human Osteoclast Ontogeny and Pathological Bone Resorption. Histol. Histopathol. 14, 635-647 (1999).

157. Olivier, V., Duval, J.L., Hindié, M., Pouletaut, P., Nagel, M.D.: Comparative Particle-Induced Cytotoxicity toward Macrophages and Fibroblasts. Cell. Biol. Toxicol. 19, 145-159 (2003).

158. Hunter, A., Archer, C.W., Walkers, P.S., Blunn, G.W.: Attachment and Proliferation of Osteoblasts and Fibroblasts on Biomaterials for Orthopaedic Use. Biomaterials. 16, 287-295 (1995).

159. Northup, S.J.: Current Problems Associated with Toxicity Evaluation of Medical Device Materials and Future Research Needs. Toxicol. Sci. 13, 196-204 (1989).

160. Martinez, M.E., Medina, S., del Campo, M.T., Garcia, J.A., Rodrigo, A., Munuera, L.: Effect of Polyethylene Particles on Human Osteoblastic Cell Growth. Biomaterials. 19, 183-187 (1998).

161. Voronov, I., Santerre, J.P., Hinek, A., Callahan, J.W., Sandhu, J., Boynton, E.L.: Macrophage Phagocytosis of Polyethylene Particulate In Vitro. J. Biomed. Mater. Res. 39, 40-51 (1998).

162. Catelas, I., Huk, O.L., Petit, A., Zukor, D.J., Marchand, R., Yahia, L.: Flow Cytometric Analysis of Macrophage Response to Ceramic and Polyethylene Particles: Effects of Size, Concentration, and Composition. J. Biomed. Mater. Res. 41, 600-607 (1998). 
163. Catelas, I., Petit, A., Marchand, R., Zukor, D.J., Yahia, L., Huk, O.L.: Cytotoxicity and Macrophage Cytokine Release Induced by Ceramic and Polyethylene Particles In Vitro. J. Bone Joint Surg. Br. 81, 516-521 (1999).

164. Kimble, R.B., Srivastava, S., Ross, F.P., Matayoshi, A., Pacifici, R.: Estrogen Deficiency Increases the Ability of Stromal Cells to Support Murine Osteoclastogenesis Via an Interleukin-1-and Tumor Necrosis Factor- Mediated Stimulation of Macrophage Colony-Stimulating Factor Production. J. Biol. Chem. 271, 28890-28897 (1996).

165. Kong, Y.Y., Feige, U., Sarosi, I., Bolon, B., Tafuri, A., Morony, S., Capparelli, C., Li, J., Elliott, R., McCabe, S., Wong, T., Campagnuolo, G., Moran, E., Bogoch, E.R., Van, G., Nguyen, L.T., Ohashi, P.S., Lacey, D.L., Fish, E., Boyle, W.J., Penninger, J.M.: Activated T cells Regulate Bone Loss and Joint Destruction in Adjuvant Arthritis Through Osteoprotegerin Ligand. Nature. 18, 304-309 (1999).

166. Teitelbaum, S.L.: Osteoclasts, Integrins, and Osteoporosis. J. Bone Miner. Metabol. 18, 344-349 (2000).

167. Nakamura, T., Imai, Y., Matsumoto, T., Sato, S., Takeuchi, K., Igarashi, K., Harada, Y., Azuma, Y., Krust, A., Yamamoto, Y., Nishina, H., Takeda, S., Takayanagi, H., Metzger, D., Kanno, J., Takaoka, K., Martin, T.J., Chambon, P., Kato, S.: Estrogen Prevents Bone Loss via Estrogen Receptor $\alpha$ and Induction of Fas Ligand in Osteoclasts. Cell. 130, 811-823 (2007).

168. Kimble, R.B., Matayoshi, A.B., Vannice, J.L., Kung, V.T., Williams, C., Pacifici, R.: Simultaneous Block of Interleukin-1 and Tumor Necrosis Factor is Required to Completely Prevent Bone Loss in the Early Postovariectomy Period. Endocrinology. 136, 3054-6061 (1995).

169. Lerner, U.H.: Inflammation-Induced Bone Remodeling in Periodontal Disease and the Influence of Post-Menopausal Osteoporosis. J. Dent. Res. 85, 596-607 (2006).

170. Lorenzo, J.: Interactions Between Immune and Bone Cells: New Insights With Many Remaining Questions. J. Clin. Invest. 106, 749-752 (2000). 